\title{
Differentiability of the Evolution Map and Mackey Continuity
}

\author{
Maximilian Hanusch* \\ Institut für Mathematik \\ Universität Paderborn \\ Warburger Straße 100 \\ 33098 Paderborn \\ Germany
}

September 6, 2019

\begin{abstract}
We solve the differentiability problem for the evolution map in Milnor's infinite dimensional setting. We first show that the evolution map of each $C^{k}$-semiregular Lie group $G$ (for $k \in$ $\mathbb{N} \sqcup\{$ lip, $\infty\}$ ) admits a particular kind of sequentially continuity - called Mackey k-continuity. We then prove that this continuity property is strong enough to ensure differentiability of the evolution map. In particular, this drops any continuity presumptions made in this context so far. Remarkably, Mackey k-continuity arises directly from the regularity problem itself, which makes it particular among the continuity conditions traditionally considered. As an application of the introduced notions, we discuss the strong Trotter property in the sequentially-, and the Mackey continuous context. We furthermore conclude that if the Lie algebra of $G$ is a Fréchet space, then $G$ is $C^{k}$-semiregular (for $k \in \mathbb{N} \sqcup\{\infty\}$ ) if and only if $G$ is $C^{k}$-regular.
\end{abstract}

\section{Contents}

1 Introduction $\quad 2$

2 Preliminaries $\quad 5$

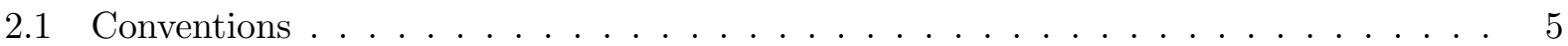

2.1 .1 Sets of Curves . . . . . . . . . . . . . . . . . 6

2.1 .2 Lie Groups . . . . . . . . . . . . . . . . . . 7 .

2.1 .3 Uniform Limits . . . . . . . . . . . . . . . . . . . . . . 7

2.2 The Evolution Map . . . . . . . . . . . . . . . . . . . . . . 7

2.2 .1 Basic Definitions . . . . . . . . . . . . . . . . . . . 8

2.2 .2 The Product Integral . . . . . . . . . . . . . . . . . 8

2.2 .3 The Exponential Map . . . . . . . . . . . . . . . . . . . 9

2.2.4 Standard Topologies . . . . . . . . . . . . . . . . . . 9

2.3 The Riemann Integral $\ldots \ldots \ldots$

2.4 Standard Facts and Estimates . . . . . . . . . . . . . . . . . . . . 10

2.5 Continuity Statements . . . . . . . . . . . . . . . . . . . 12

*mhanusch@math.upb.de 
3 Auxiliary Results $\quad \mathbf{1 2}$

3.1 Sets of Curves . . . . . . . . . . . . . . . . . . . . . 13

3.2 Weak Continuity . . . . . . . . . . . . . . . . . . . 14

3.3 Mackey Continuity . . . . . . . . . . . . . . . . . . . . . 15

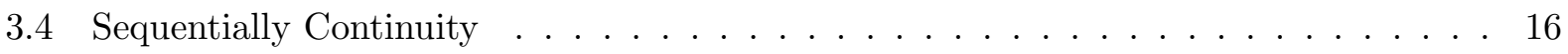

3.5 Piecewise Integrable Curves . . . . . . . . . . . . . . . . . . . 17

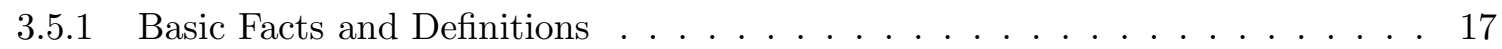

3.5.2 A Continuity Statement . . . . . . . . . . . . . . . . . 18

4 Mackey Continuity 19

4.1 Some Estimates . . . . . . . . . . . . . . . . . . . . . . . . . . . . 19

4.2 A Construction . . . . . . . . . . . . . . . . . . . . . . 20

4.3 Proof of Theorem $1 . \ldots \ldots \ldots \ldots \ldots \ldots \ldots \ldots$

5 The Strong Trotter Property $\quad 22$

6 Differentiation $\quad 2$

6.1 Some Technical Statements . . . . . . . . . . . . . . . . . . . . . 24

6.2 The Differential of the Evolution Map . . . . . . . . . . . . . . . 25

6.2 .1 The Generic Case . . . . . . . . . . . . . . . . . . . . . 27

6.2 .2 The Exponential Map . . . . . . . . . . . . . . . . . 28

6.3 Integrals with Parameters . . . . . . . . . . . . . . . . . 28

6.4 Differentiation at Zero . . . . . . . . . . . . . . . . . . . 30

7 Extension: The Metrizable Category 3

\section{Introduction}

In 1983 Milnor introduced his regularity concept [15] as a tool to extend proofs of fundamental Lie theoretical facts to infinite dimensions. Specifically, he adapted (and weakened) the regularity concept introduced in 1982 by Omori, Maeda, Yoshioka and Kobayashi for Fréchet Lie groups [20] to such Lie groups that are modeled over complete Hausdorff locally convex vector spaces. Then, he used this notion to prove the integrability of Lie algebra homomorphisms to Lie group homomorphisms under certain regularity and connectedness presumptions. In this paper, we work in the slightly more general setting introduced by Glöckner in [2] - specifically meaning that any completeness presumption on the modeling space is dropped 1$]$

Roughly speaking, regularity is concerned with definedness, continuity, and smoothness of the evolution map (product integral) - a notion that naturally generalizes the concept of the Riemann integral for curves in locally convex vector spaces, to infinite dimensional Lie groups (Lie algebra valued curves are thus integrated to Lie group elements). For instance, the exponential map of a Lie group is the restriction of the evolution map to constant curves; and, given a principal fibre bundle, then holonomies are evolutions of such Lie algebra valued curves that are pairings of smooth connections with derivatives of curves in the base manifold of the bundle. Although individual arguments show that the generic infinite dimensional Lie group is $C^{\infty}$-regular or stronger, only recently general regularity criteria had been found [3,7, 16]. Differentiability of the evolution map (hence, of the exponential map) is one of the key components of the regularity problem. In [3, 7, this issue had been discussed in the standard topological context - implicitly meaning that

${ }^{1}$ Confer also 16, 17 for an introduction to this area. To prevent confusion, we additionally remark that Milnor's definition of an infinite dimensional manifold $M$ involves the requirement that $M$ is a regular topological space, i.e., fulfills the separation axioms $T_{2}, T_{3}$. Deviating from that, in [2], only the $T_{2}$ property of $M$ is explicitly presumed This, however, makes no difference in the Lie group case, because topological groups are automatically $T_{3}$. 
continuity of the evolution maps w.r.t. to the $C^{k}$-topology was presumed 2 In this paper, we solve the differentiability problem in full generality, as we drop any continuity presumption made in this context so far. The results obtained in particular imply that if the Lie group is modeled over a Fréchet space, with evolution map defined on all $C^{k}$-curves (the Lie group is $C^{k}$-semiregular), then the evolution map is automatically smooth w.r.t. to the $C^{k}$-topology (the Lie group is $C^{k}$-regular). We furthermore generalize the results obtained in [4,8] concerning the strong Trotter property by weakening the continuity presumptions made there.

More specifically, let $G$ denote an infinite dimensional Lie group as defined in [2] that is modeled over the Hausdorff locally convex vector space $E$. We let $\mathfrak{g}$ denote the Lie algebra of $G$; as well as $\mathrm{d}_{q} \mathrm{R}_{g}$ the differential of the right translation $\mathrm{R}_{g}: G \ni h \mapsto h \cdot g$ by $g \in G$, at the point $q \in G$. We furthermore define (right logarithmic derivative)

$$
C^{0}([0,1], \mathfrak{g}) \ni \delta^{r}(\mu):=\mathrm{d}_{\mu} \mathrm{R}_{\mu^{-1}}(\dot{\mu}) \quad \forall \mu \in C^{1}([0,1], G)
$$

as well as D $:=\left\{\delta^{r}(\mu) \mid \mu \in C^{1}([0,1], G)\right\}$ and $C_{*}^{1}([0,1], G):=\left\{\mu \in C^{1}([0,1], G) \mid \mu(0)=e\right\}$. The evolution maps are given by

$$
\begin{aligned}
\text { Evol: } \mathrm{D} \ni \delta^{r}(\mu) & \mapsto \mu \cdot \mu^{-1}(0) \in C_{*}^{1}([0,1], G) \\
\text { evol: } \mathrm{D} \ni \phi & \mapsto \operatorname{Evol}(\phi)(1) \in G \\
\text { as well as } & \\
\text { Evol }_{\mathrm{k}}:=\operatorname{Evol}_{D_{\mathrm{k}}} \quad & \text { and } \quad \operatorname{evol}_{\mathrm{k}}:=\left.\operatorname{evol}\right|_{D_{\mathrm{k}}},
\end{aligned}
$$

with $\mathrm{D}_{\mathrm{k}}:=\mathrm{D} \cap C^{k}([0,1], \mathfrak{g})$ for each $k \in \mathbb{N} \sqcup\{$ lip, $\infty, \mathrm{c}\} 3$ We say that $G$ is $C^{k}$-semiregular if $C^{k}([0,1], \mathfrak{g}) \subseteq \mathrm{D}$ holds; hence, if each $\phi \in C^{k}([0,1], \mathfrak{g})$ admits a (necessarily unique) solution $\mu \in C_{*}^{1}([0,1], G)$ to the differential equation $\delta^{r}(\mu)=\phi$. It was shown in [3] (cf. Theorem E in [3]) that if $G$ is $C^{k}$-semiregular for $k \in \mathbb{N} \sqcup\{\infty\}$, then Evol $_{\mathrm{k}}$ (thus, evol $\mathrm{k}_{\mathrm{k}}$ ) is smooth if and only if evol $\mathrm{k}_{\mathrm{k}}$

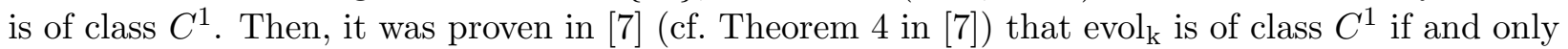
if it is continuous, with $\mathfrak{g}$ Mackey complete for $k \in \mathbb{N}_{\geq 1} \sqcup\{$ lip, $\infty\}$ (as well as integral complete for $k=0$ ). All these statement have been established in the standard topological context - specifically meaning that evol $_{\mathrm{k}}\left(\right.$ and $\mathrm{Evol}_{\mathrm{k}}$ ) was presumed to be continuous w.r.t. the $C^{k}$-topology. In this paper, we more generally show that, cf. (the more comprehensive) Theorem 2 in Sect. 6.2.1

Theorem A. Suppose that $G$ is $C^{k}$-semiregular for $k \in \mathbb{N} \sqcup\{$ lip, $\infty\}$. Then, evol $_{\mathrm{k}}$ is differentiable

$$
\text { - for } k=0 \quad \text { if and only if } \mathfrak{g} \text { is integral complete. }
$$

- for $k \in \mathbb{N}_{\geq 1} \sqcup\{\infty\}$ if and only if $\mathfrak{g}$ is Mackey complete.

In this case, evol $_{\mathrm{k}}$ is differentiable, with 4

$$
\mathrm{d}_{\phi} \operatorname{evol}_{\mathrm{k}}(\psi)=\mathrm{d}_{e} \mathrm{~L}_{\int \phi}\left(\int \operatorname{Ad}_{\left[\int_{r}^{s} \phi\right]^{-1}}(\psi(s)) \mathrm{d} s\right) \quad \forall \phi, \psi \in C^{k}\left(\left[r, r^{\prime}\right], \mathfrak{g}\right) .
$$

This theorem will be derived from significantly more fundamental results established in this paper: Let $\Xi: \mathcal{U} \rightarrow \mathcal{V} \subseteq E$ be a fixed chart around $e$, and $\mathfrak{P}$ the system of continuous seminorms on $E$. A pair $(\phi, \psi) \in C^{0}([0,1], \mathfrak{g}) \times C^{0}([0,1], \mathfrak{g})$ is said to be

- admissible if $\phi+(-\delta, \delta) \cdot \psi \subseteq \mathrm{D}$ holds for some $\delta>0$,

- regular if it is admissible, with

$$
\lim _{h \rightarrow 0}^{\infty} \Xi\left(\operatorname{Evol}(\phi)^{-1} \cdot \operatorname{Evol}(\phi+h \cdot \psi)\right)=0 .
$$

\footnotetext{
${ }^{2}$ The $C^{k}$-topology is recalled in Sect. 2.2.4 and, the evolution maps are defined below.

${ }^{3}$ Here, $C^{\text {lip }}([0,1], \mathfrak{g})$ denotes the set of Lipschitz curves, and $C^{\mathrm{c}}([0,1], \mathfrak{g})$ denotes the set of constant curves.

${ }^{4}$ Notably, this formula is well known from the finite dimensional context (cf., e.g., the proof of (1.13.4) Proposition in [1]), and also for regular Lie groups in the convenient setting [1].
} 
Here, the limit is understood to be uniform - In general, we write $\lim _{h \rightarrow 0}^{\infty} \alpha=\beta$ for $\alpha:(-\delta, 0) \sqcup$ $(0, \delta) \times[0,1] \rightarrow E$ with $\delta>0$ and $\beta:[0,1] \rightarrow \bar{E}$ if

$$
\lim _{h \rightarrow 0} \sup \{\overline{\mathfrak{p}}(\alpha(h, t)-\beta(t)) \mid t \in[0,1]\}=0 \quad \forall \mathfrak{p} \in \mathfrak{P}
$$

holds, where $\overline{\mathfrak{p}}: \bar{E} \rightarrow \mathbb{R}_{\geq 0}$ denotes the continuous extension of the seminorm $\mathfrak{p} \in \mathfrak{P}$ to the completion $\bar{E}$ of $E$. Then, the first result we want to mention is, cf. Proposition 3 in Sect. 6.2

Proposition B. Suppose that $(\phi, \psi)$ is admissible.

1) The pair $(\phi, \psi)$ is regular if and only if we have

$$
\lim _{h \rightarrow 0}^{\infty} 1 / h \cdot \Xi\left(\operatorname{Evol}(\phi)^{-1} \cdot \operatorname{Evol}(\phi+h \cdot \psi)\right)=\int_{r}^{\bullet}\left(\mathrm{d}_{e} \Xi \circ \operatorname{Ad}_{\operatorname{Evol}(\phi)(s)^{-1}}\right)(\psi(s)) \mathrm{d} s \in \bar{E} .
$$

2) If $(\phi, \psi)$ is regular, then $(-\delta, \delta) \ni h \mapsto \operatorname{evol}(\phi+h \cdot \psi) \in G$ is differentiable at $h=0$ (for $\delta>0$ suitably small) if and only if $\int \operatorname{Ad}_{\mathrm{Evol}(\phi)(s)^{-1}}(\psi(s)) \mathrm{d} s \in \mathfrak{g}$ holds. In this case, we have

$$
\left.\frac{\mathrm{d}}{\mathrm{d} h}\right|_{h=0} \operatorname{evol}(\phi+h \cdot \psi)=\mathrm{d}_{e} \mathrm{~L}_{\mathrm{evol}(\phi)}\left(\int \operatorname{Ad}_{\operatorname{Evol}(\phi)(s)^{-1}}(\psi(s)) \mathrm{d} s\right) .
$$

Evidently, each $(\phi, \psi) \in C^{k}([0,1], \mathfrak{g}) \times C^{k}([0,1], \mathfrak{g})$ is admissible if and only if $G$ is $C^{k}$-semiregular. In Sect. 4, we furthermore prove that, cf. Theorem 1 in Sect. 4

Theorem C. If $G$ is $C^{k}$-semiregular for $k \in \mathbb{N} \sqcup\{$ lip, $\infty\}$, then $G$ is Mackey k-continuous.

Here, Mackey k-continuity is a specific kind of sequentially continuity (cf. Sect. 3.3) that, in particular, implies that each admissible $(\phi, \psi) \in C^{k}([0,1], \mathfrak{g}) \times C^{k}([0,1], \mathfrak{g})$ is regular (cf. Lemma 15 in Sect. 3.3) - Theorem A thus follows immediately from Proposition B and Theorem C. We will conclude from Theorem $\mathrm{C}$ and Theorem 4 in [7] that, cf. Corollary 7 in Sect. 7

Corollary D. Suppose that $\mathfrak{g}$ is a Fréchet space; and let $k \in \mathbb{N} \sqcup\{\infty\}$ be fixed. Then, $G$ is $C^{k}$-regular if and only if $G$ is $C^{k}$-semiregular.

Now, Proposition B is actually a consequence of a more fundamental differentiability result (Proposition 2 in Sect. 6) that we will also use to generalize Theorem 5 in [7]. Specifically, we will prove that, cf. Theorem 3 in Sect. 6.3

Theorem E. Suppose that $G$ is Mackey k-continuous for $k \in \mathbb{N} \sqcup\{$ lip, $\infty, \mathrm{c}\}$ - additionally abelian if $k=\mathrm{c}$ holds. Let $\Phi: I \times[0,1] \rightarrow \mathfrak{g}\left(I \subseteq \mathbb{R}\right.$ open) be given with $\Phi(z, \cdot) \in \mathrm{D}_{\mathrm{k}}$ for each $z \in I$. Then,

$$
\lim _{h \rightarrow 0}^{\infty} 1 / h \cdot \Xi\left(\operatorname{Evol}(\Phi(x, \cdot))^{-1} \cdot \operatorname{Evol}(\Phi(x+h, \cdot))\right)=\int_{r}^{\bullet}\left(\mathrm{d}_{e} \Xi \circ \operatorname{Ad}_{\operatorname{Evol}(\Phi(x, \cdot))(s)}\right)\left(\partial_{z} \Phi(x, s)\right) \mathrm{d} s \in \bar{E}
$$

holds for $x \in I$, provided that

a) We have $\left(\partial_{z} \Phi\right)(x, \cdot) \in C^{k}([0,1], \mathfrak{g})$.

b) For each $\mathfrak{p} \in \mathfrak{P}$ and $\mathrm{s} \preceq k$ 局 there exists $L_{\mathfrak{p}, \mathrm{s}} \geq 0$, and $I_{\mathfrak{p}, \mathrm{s}} \subseteq I$ open with $x \in I_{\mathfrak{p}, \mathrm{s}}$, such that

$$
1 /|h| \cdot \mathfrak{p p}_{\infty}^{\mathrm{s}}(\Phi(x+h, \cdot)-\Phi(x, \cdot)) \leq L_{\mathfrak{p}, \mathrm{s}} \quad \forall h \in \mathbb{R}_{\neq 0} \text { with } x+h \in I_{\mathfrak{p}, \mathrm{s}} .
$$

In particular, we have

$$
\left.\frac{\mathrm{d}}{\mathrm{d} h}\right|_{h=0} \operatorname{evol}(\Phi(x+h, \cdot))=\mathrm{d}_{e} \mathrm{~L}_{\operatorname{evol}(\Phi(x, \cdot))}\left(\int \operatorname{Ad}_{\operatorname{Evol}(\Phi(x, \cdot))(s)}\left(\partial_{z} \Phi(x, s)\right) \mathrm{d} s\right)
$$

if and only if the Riemann integral on the right side exists in $\mathfrak{g}$.

\footnotetext{
${ }^{5}$ This means $\mathrm{s}=\operatorname{lip}$ for $k=\operatorname{lip}, \mathrm{s}=0$ for $k=0,0 \leq \mathrm{s} \leq k$ for $k \in \mathbb{N}$, and $\mathrm{s} \in \mathbb{N}$ for $k=\infty$. The corresponding seminorms $\cdot \mathfrak{p}_{\infty}^{\mathrm{s}}$ are defined in Sect. 2.1.1
} 
We explicitly recall at this point that, by Theorem $\mathbb{C}$, for $k \in \mathbb{N} \sqcup\{$ lip, $\infty\}$, Mackey k-continuity is automatically given if $G$ is $C^{k}$-semiregular. Finally, let exp: $\mathfrak{g} \supseteq \operatorname{dom}[\exp ] \rightarrow G$ denote the exponential map of $G$; and recall that a Lie group $G$ is said to have the strong Trotter property [4, 8, 13, 19] if for each $\mu \in C_{*}^{1}([0,1], G)$ with $\dot{\mu}(0) \in \operatorname{dom}[\exp ]$, we have

$$
\lim _{n} \mu(\tau / n)^{n}=\exp (\tau \cdot \dot{\mu}(0)) \quad \forall \tau \in[0, \ell]
$$

uniformly $\sqrt{6}$ for each $\ell>0$. As already figured out in [4, the strong Trotter property implies the strong commutator property; and, also the Trotter and the commutator property that are relevant, e.g., in representation theory of infinite dimensional Lie groups [19]. Now, Theorem I in [4] states that $G$ has the strong Trotter property if $G$ is $R$-regular. This was generalized in [8] to the locally $\mu$-convex case (hence, the case where evol $_{0}$ is $C^{0}$-continuous on its domain, cf. Theorem 1 in [7]). In this paper, we go a step further, as we show (cf. Proposition 1 in Sect. 5) that $G$ has the

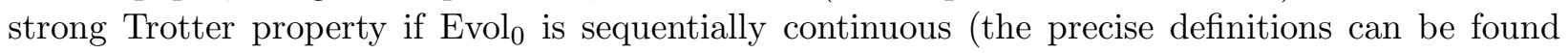
in Sect. 3.4); which is much weaker than locally $\mu$-convexity (provided that $\mathfrak{g}$ is not metrizable, of course). We furthermore show in Proposition 1 that (1) holds for each $\mu \in C_{*}^{1}([0,1], G)$ with $\dot{\mu}(0) \in \operatorname{dom}[\exp ]$ and $\delta^{r}(\mu) \in C^{\operatorname{lip}}([0,1], \mathfrak{g})$ if $\operatorname{Evol}_{0}$ is Mackey continuous, the latter condition being even less restrictive than sequentially continuity.

This paper is organized as follows:

- In Sect. 2, we provide the basic definitions; and discuss the most elementary properties of the core mathematical objects of this paper.

- In Sect. 3, we discuss the continuity notions considered in this paper.

- In Sect. 4, we prove Theorem 1 (i.e., Theorem C).

- In Sect. 5, we discuss the strong Trotter property in the sequentially/Mackey continuous context.

- In Sect. 6, we establish the differentiability results for the evolution map.

- In Sect. 7, we prove Corollary 7 (i.e., Corollary D).

\section{Preliminaries}

In this section, we fix the notations, and discuss the properties of the product integral (evolution map) that we will need in the main text. The proofs of the facts mentioned but not verified in this section, can be found, e.g., in Sect. 3 and Sect. 4 in [7].

\subsection{Conventions}

In this paper, Manifolds and Lie groups are always understood to be in the sense of [2]; in particular, smooth, Hausdorff, and modeled over a Hausdorff locally convex vector space 7 If $f: M \rightarrow N$ is a $C^{1}$-map between the manifolds $M$ and $N$, then $\mathrm{d} f: T M \rightarrow T N$ denotes the corresponding tangent map between their tangent manifolds - we write $\mathrm{d}_{x} f \equiv \mathrm{d} f(x, \cdot): T_{x} M \rightarrow T_{f(x)} N$ for each $x \in M$. By an interval, we understand a non-empty, non-singleton connected subset $D \subseteq \mathbb{R}$. The set of all compact intervals is denoted by $\mathfrak{K}=\left\{\left[r, r^{\prime}\right] \subseteq \mathbb{R} \mid r<r^{\prime}\right\}$. We furthermore let $\mathcal{D}_{\delta}:=(-\delta, 0) \sqcup(0, \delta)$ for each $\delta>0$. A curve is a continuous map $\gamma: D \rightarrow M$ for a manifold $M$ and an interval $D \subseteq \mathbb{R}$. If $D \equiv I$ is open, then $\gamma$ is said to be of class $C^{k}$ for $k \in \mathbb{N} \sqcup\{\infty\}$ if it is of class $C^{k}$ when considered

${ }^{6}$ Thus, for each neighbourhood $U \subseteq G$ of $e$, there exists some $n_{U} \in \mathbb{N}$ with $\exp (-\tau \cdot \dot{\mu}(0)) \cdot \mu(\tau / n)^{n} \in U$ for each $n \geq n_{U}$ and $\tau \in[0, \ell]$.

${ }^{7}$ We explicitly refer to Definition 3.1 and Definition 3.3 in 2]. A review of the corresponding differential calculus including the standard differentiation rules used in this paper - can be found, e.g., in Appendix A.1 that essentially equals Sect. 3.3.1 in [7. 
as a map between the manifolds $I$ and $M$. If $D$ is an arbitrary interval, then $\gamma$ is said to be of class $C^{k}$ for $k \in \mathbb{N} \sqcup\{\infty\}$ if $\gamma=\left.\gamma^{\prime}\right|_{D}$ holds for a $C^{k}$-curve $\gamma^{\prime}: I \rightarrow M$ that is defined on an open interval $I$ containing $D$ - we write $\gamma \in C^{k}(D, M)$ in this case. If $\gamma: D \rightarrow M$ is of class $C^{1}$, then we denote the corresponding tangent vector at $\gamma(t) \in M$ by $\dot{\gamma}(t) \in T_{\gamma(t)} M$. The above conventions also hold if $M \equiv F$ is a Hausdorff locally convex vector space with system of continuous seminorms $\mathfrak{Q}$. In this case, we let $\bar{F}$ denote the completion of $F$; as well as $\overline{\mathfrak{q}}: \bar{F} \rightarrow \mathbb{R}_{>0}$ the continuous extension of $\mathfrak{q}$ to $\bar{F}$, for each $\mathfrak{q} \in \mathfrak{Q}$. We furthermore define

$$
\mathrm{B}_{\mathfrak{q}, \varepsilon}:=\{X \in F \mid \mathfrak{q}(X)<\varepsilon\} \quad \text { as well as } \quad \bar{B}_{\mathfrak{q}, \varepsilon}:=\{X \in F \mid \mathfrak{q}(X) \leq \varepsilon\}
$$

for all $\mathfrak{q} \in \mathfrak{Q}$ and $\varepsilon>0$. If $X, Y$ are sets, then $\operatorname{Map}(X, Y) \equiv Y^{X}$ denotes the set of all mappings $X \rightarrow Y$.

\subsubsection{Sets of Curves}

Let $F$ be a Hausdorff locally convex vector space with system of continuous seminorms $\mathfrak{Q}$.

- By $C^{\operatorname{lip}}\left(\left[r, r^{\prime}\right], F\right)$ we denote the set of all Lipschitz curves on $\left[r, r^{\prime}\right] \in \mathfrak{K}$; i.e., all curves $\gamma:\left[r, r^{\prime}\right] \rightarrow$ $F$, such that

$$
\operatorname{Lip}(\mathfrak{q}, \gamma):=\sup \left\{\frac{\mathfrak{q}\left(\gamma(t)-\gamma\left(t^{\prime}\right)\right)}{\left|t-t^{\prime}\right|} \mid t, t^{\prime} \in\left[r, r^{\prime}\right], t \neq t^{\prime}\right\} \in \mathbb{R}_{\geq 0}
$$

exists for each $\mathfrak{q} \in \mathfrak{Q}$ - i.e., we have

$$
\mathfrak{q}\left(\gamma(t)-\gamma\left(t^{\prime}\right)\right) \leq \operatorname{Lip}(\mathfrak{q}, \gamma) \cdot\left|t-t^{\prime}\right| \quad \forall t, t^{\prime} \in\left[r, r^{\prime}\right], \mathfrak{q} \in \mathfrak{Q} .
$$

- By $C^{\mathrm{c}}\left(\left[r, r^{\prime}\right], F\right)$ we denote the set of all constant curves on $\left[r, r^{\prime}\right] \in \mathfrak{K}$; i.e., all curves of the form

$$
\gamma_{X}:\left[r, r^{\prime}\right] \rightarrow F, \quad t \mapsto X
$$

for some $X \in F$.

We define $c+1:=\infty, \infty+1:=\infty$, lip $+1:=1$; as well as

$$
\begin{array}{lll}
\mathfrak{q}_{\infty}^{\operatorname{lip}}(\gamma):=\max \left(\mathfrak{q}_{\infty}(\gamma), \operatorname{Lip}(\mathfrak{q}, \gamma)\right) & & \forall \gamma \in C^{\operatorname{lip}}\left(\left[r, r^{\prime}\right], F\right) \\
\mathfrak{q}_{\infty}^{\mathrm{s}}(\gamma):=\sup \left\{\mathfrak{q}\left(\gamma^{(m)}(t)\right) \mid 0 \leq m \leq \mathrm{s}, t \in\left[r, r^{\prime}\right]\right\} & & \forall \gamma \in C^{k}\left(\left[r, r^{\prime}\right], F\right) \\
\mathfrak{q}_{\infty}(\gamma):=\mathfrak{q}_{\infty}^{0}(\gamma) & & \forall \gamma \in C^{0}\left(\left[r, r^{\prime}\right], F\right)
\end{array}
$$

for each $\mathfrak{q} \in \mathfrak{Q}, k \in \mathbb{N} \sqcup\{\infty, \mathrm{c}\}, \mathrm{s} \preceq k$, and $\left[r, r^{\prime}\right] \in \mathfrak{K}-$ Here, $s \preceq k$ means

- $\mathrm{s}=\operatorname{lip} \quad$ for $k=\operatorname{lip}$,

- $\mathbb{N} \ni \mathrm{s} \leq k$ for $k \in \mathbb{N}$,

- $\mathrm{s} \in \mathbb{N}$ for $k=\infty$,

- $\mathrm{s}=0 \quad$ for $k=\mathrm{c}$.

The $C^{k}$-topology on $C^{k}\left(\left[r, r^{\prime}\right], F\right)$ for $k \in \mathbb{N} \sqcup\{$ lip,, , c $\}$ is the Hausdorff locally convex topology that is generated by the seminorms $\mathfrak{q}_{\infty}^{\mathrm{s}}$ for all $\mathfrak{q} \in \mathfrak{Q}$ and $\mathrm{s} \preceq k$.

Remark 1. In the Lipschitz case, the above conventions deviate from the conventions used, e.g., in [7, 9] as there the $\mathfrak{p}_{\infty}$-seminorms, i.e., the $C^{0}$-topology is considered on $C^{\operatorname{lip}}\left(\left[r, r^{\prime}\right], F\right)$.

Finally, we let $\mathrm{CP}^{0}\left(\left[r, r^{\prime}\right], F\right)$ denote the set of piecewise $C^{0}$-curves on $\left[r, r^{\prime}\right] \in \mathfrak{K}$; i.e., all $\gamma:\left[r, r^{\prime}\right] \rightarrow$ $F$, such that there exist $r=t_{0}<\ldots<t_{n}=r^{\prime}$ and

$$
\gamma[p] \in C^{0}\left(\left[t_{p}, t_{p+1}\right], F\right) \quad \text { with }\left.\quad \gamma\right|_{\left(t_{p}, t_{p+1}\right)}=\left.\gamma[p]\right|_{\left(t_{p}, t_{p+1}\right)} \quad \text { for } \quad p=0, \ldots, n-1 .
$$




\subsubsection{Lie Groups}

In this paper, $G$ will always denote an infinite dimensional Lie group in the sense of [2] (cf. Definition 3.1 and Definition 3.3 in [2]) that is modeled over the Hausdorff locally convex vector space $E$, with corresponding system of continuous seminorms $\mathfrak{P}$. We denote the Lie algebra of $G$ by $(\mathfrak{g},[\cdot, \cdot])$, fix a chart

$$
\Xi: G \supseteq U \rightarrow \mathcal{V} \subseteq E
$$

with $\mathcal{V}$ convex, $e \in \mathcal{U}$ and $\Xi(e)=0$; and define

$$
\mathfrak{p}:=\mathfrak{p} \circ \mathrm{d}_{e} \Xi \quad \forall \mathfrak{p} \in \mathfrak{P} .
$$

We let $\mathrm{m}: G \times G \rightarrow G$ denote the Lie group multiplication, $\mathrm{R}_{g}:=\mathrm{m}(\cdot, g)$ the right translation by $g \in G$, inv : $G \ni g \mapsto g^{-1} \in G$ the inversion, and Ad: $G \times \mathfrak{g} \rightarrow \mathfrak{g}$ the adjoint action - i.e., we have

$$
\operatorname{Ad}(g, X) \equiv \operatorname{Ad}_{g}(X):=\operatorname{d}_{e} \operatorname{Conj}_{g}(X) \quad \text { with } \quad \operatorname{Conj}_{g}: G \ni h \mapsto g \cdot h \cdot g^{-1} \in G
$$

for each $g \in G$ and $X \in \mathfrak{g}$. We furthermore recall the product rule

$$
\mathrm{d}_{(g, h)} \mathrm{m}(v, w)=\mathrm{d}_{g} \mathrm{R}_{h}(v)+\mathrm{d}_{h} \mathrm{~L}_{g}(w) \quad \forall g, h \in G, v \in T_{g} G, w \in T_{h} G .
$$

\subsubsection{Uniform Limits}

Let $\mu \in \operatorname{Map}\left(\left[r, r^{\prime}\right], G\right),\left\{\mu_{n}\right\}_{n \in \mathbb{N}} \subseteq \operatorname{Map}\left(\left[r, r^{\prime}\right], G\right)$, and $\left\{\mu_{h}\right\}_{h \in \mathcal{D}_{\delta}} \subseteq \operatorname{Map}\left(\left[r, r^{\prime}\right], G\right)$ for $\delta>0$ be given. We write

- $\lim _{n}^{\infty} \mu_{n}=\mu \quad$ if $\quad$ for each open neighbourhood $U \subseteq G$ of $e$, there exists some $n_{U} \in \mathbb{N}$ with $\mu^{-1} \cdot \mu_{n} \in U$ for each $n \geq n_{U}$.

- $\lim _{h \rightarrow 0}^{\infty} \mu_{h}=\mu$ if for each open neighbourhood $U \subseteq G$ of $e$, there exists some $0<\delta_{U}<\delta$ with $\mu^{-1} \cdot \mu_{h} \in U$ for each $h \in \mathcal{D}_{\delta_{U}}$.

Evidently, then we have

Lemma 1. Suppose $\delta>0$ and $\left\{\mu_{h}\right\}_{h \in \mathcal{D}_{\delta}} \subseteq C^{0}\left(\left[r, r^{\prime}\right], G\right)$ are given. If $\lim _{n}^{\infty} \mu_{h_{n}}=e$ holds for each sequence $\mathcal{D}_{\delta} \supseteq\left\{h_{n}\right\}_{n \in \mathbb{N}} \rightarrow 0$, then we have $\lim _{h \rightarrow 0}^{\infty} \mu_{h}=e$.

Proof. If the claim is wrong, then there exists a neighbourhood $U \subseteq G$ of $e$, such that the following holds: For each $n \in \mathbb{N}$, there exists some $h_{n} \neq 0$ with $\left|h_{n}\right| \leq \frac{1}{n}$ as well as some $\tau_{n} \in\left[r, r^{\prime}\right]$, such that $\mu^{-1}\left(\tau_{n}\right) \cdot \mu_{h_{n}}\left(\tau_{n}\right) \notin U$ holds. Since we have $\left\{h_{n}\right\}_{n \in \mathbb{N}} \rightarrow 0$, this contradicts the presumptions.

The same conventions (and Lemma 1) also hold if $(G, \cdot) \equiv(F,+)$ is a Hausdorff locally convex vector space (or its completion) - In this case, we use the following convention:

Let $\delta>0$ and $\alpha: \mathcal{D}_{\delta} \times\left[r, r^{\prime}\right] \rightarrow F$ be given, with $\alpha(h, \cdot) \in \operatorname{Map}\left(\left[r, r^{\prime}\right], F\right)$ for each $h \in \mathcal{D}_{\delta}$. Then, for $\beta \in \operatorname{Map}\left(\left[r, r^{\prime}\right], F\right)$, we write

$$
\left.\frac{\mathrm{d}}{\mathrm{d} h}\right|_{h=0} ^{\infty} \alpha=\beta \quad \stackrel{\text { def. }}{\Longleftrightarrow} \quad \lim _{h \rightarrow 0}^{\infty}[1 / h \cdot \alpha(h, \cdot)]=\beta .
$$

Remark 2. In this paper, the above convention will mainly be used in the following form: $F=\bar{E}$ will be the completion of a Hausdorff locally convex vector space $E$; and we will have $\alpha: \mathcal{D}_{\delta} \times\left[r, r^{\prime}\right] \rightarrow$ $E \subseteq \bar{E}$ as well as $\beta \in \operatorname{Map}\left(\left[r, r^{\prime}\right], \bar{E}\right)$.

\subsection{The Evolution Map}

In this subsection, we provide the relevant facts and definitions concerning the right logarithmic derivative and the evolution map. 


\subsubsection{Basic Definitions}

We define

$$
C_{*}^{k}\left(\left[r, r^{\prime}\right], G\right):=\left\{\mu \in C^{k}\left(\left[r, r^{\prime}\right], G\right) \mid \mu(r)=e\right\} \quad \forall\left[r, r^{\prime}\right] \in \mathfrak{K}, k \in \mathbb{N} \sqcup\{\infty\} .
$$

The right logarithmic derivative is given by

$$
\delta^{r}: C^{1}\left(\left[r, r^{\prime}\right], G\right) \rightarrow C^{0}\left(\left[r, r^{\prime}\right], \mathfrak{g}\right), \quad \mu \mapsto \mathrm{d}_{\mu} \mathrm{R}_{\mu^{-1}}(\dot{\mu})
$$

for each $\left[r, r^{\prime}\right] \in \mathfrak{K}$; and we define $\mathfrak{D}_{\left[r, r^{\prime}\right]}:=\delta^{r}\left(C^{1}\left(\left[r, r^{\prime}\right], G\right)\right)$ for each $\left[r, r^{\prime}\right] \in \mathfrak{K}$, as well as

$$
\mathfrak{D}_{\left[r, r^{\prime}\right]}^{k}:=\mathfrak{D}_{\left[r, r^{\prime}\right]} \cap C^{k}\left(\left[r, r^{\prime}\right], \mathfrak{g}\right) \quad \forall\left[r, r^{\prime}\right] \in \mathfrak{K}, k \in \mathbb{N} \sqcup\{\text { lip }, \infty, \mathrm{c}\} .
$$

Then, $\delta^{r}$ restricted to $C_{*}^{1}\left(\left[r, r^{\prime}\right], G\right)$ is injective for each $\left[r, r^{\prime}\right] \in \mathfrak{K}$; so that

$$
\text { Evol: } \bigsqcup_{\left[r, r^{\prime}\right] \in \mathfrak{K}} \mathfrak{D}_{\left[r, r^{\prime}\right]} \rightarrow \bigsqcup_{\left[r, r^{\prime}\right] \in \mathfrak{K}} C_{*}^{1}\left(\left[r, r^{\prime}\right], G\right)
$$

is well defined by

$$
\text { Evol: } \mathfrak{D}_{\left[r, r^{\prime}\right]} \rightarrow C_{*}^{1}\left(\left[r, r^{\prime}\right], G\right), \quad \delta^{r}(\mu) \mapsto \mu \cdot \mu(r)^{-1}
$$

for each $\left[r, r^{\prime}\right] \in \mathfrak{K}$. Here,

$$
\left.\operatorname{Evol}\right|_{\mathfrak{D}_{\left[r, r^{\prime}\right]}^{k}}: \mathfrak{D}_{\left[r, r^{\prime}\right]}^{k} \rightarrow C^{k+1}\left(\left[r, r^{\prime}\right], G\right)
$$

holds for each $\left[r, r^{\prime}\right] \in \mathfrak{K}$, and each $k \in \mathbb{N} \sqcup\{\operatorname{lip}, \infty, \mathrm{c}\}$.

\subsubsection{The Product Integral}

The product integral is given by

$$
\int_{s}^{t} \phi:=\operatorname{Evol}\left(\left.\phi\right|_{[s, t]}\right)(t) \in G \quad \forall[s, t] \subseteq \operatorname{dom}[\phi], \phi \in \bigsqcup_{\left[r, r^{\prime}\right] \in \mathfrak{K}} \mathfrak{D}_{\left[r, r^{\prime}\right]} ;
$$

and we let $\int \phi \equiv \int_{r}^{r^{\prime}} \phi$ as well as $\int_{c}^{c} \phi:=e$ for $\phi \in \mathfrak{D}_{\left[r, r^{\prime}\right]}$ and $c \in\left[r, r^{\prime}\right]$. Moreover, we set

$$
\left.\operatorname{evol}_{\left[r, r^{\prime}\right]}^{k} \equiv \int\right|_{\mathfrak{D}_{\left[r, r^{\prime}\right]}^{k}} \quad \forall k \in \mathbb{N} \sqcup\{\operatorname{lip}, \infty, \mathrm{c}\}, \quad\left[r, r^{\prime}\right] \in \mathfrak{K} ;
$$

and let $\operatorname{evol}_{\mathrm{k}} \equiv \operatorname{evol}_{[0,1]}^{k}$ as well as $\mathrm{D}_{\mathrm{k}} \equiv \mathfrak{D}_{[0,1]}^{k}$ for each $k \in \mathbb{N} \sqcup\{$ lip, $\infty, \mathrm{c}\}$. We furthermore let

$$
\text { evol } \equiv \operatorname{evol}_{0}: \mathrm{D} \equiv \mathrm{D}_{0} \rightarrow G \text {. }
$$

Then, we have the following elementary identities, cf., [3, 11] or Sect. 3.5.2 in [7]

a) For each $\phi, \psi \in \mathfrak{D}_{\left[r, r^{\prime}\right]}$, we have $\phi+\operatorname{Ad}_{\int_{r}^{\bullet} \phi}(\psi) \in \mathfrak{D}_{\left[r, r^{\prime}\right]}$, with

$$
\int_{r}^{t} \phi \cdot \int_{r}^{t} \psi=\int_{r}^{t} \phi+\operatorname{Ad}_{\int_{r}^{\bullet} \phi}(\psi) .
$$

b) For each $\phi, \psi \in \mathfrak{D}_{\left[r, r^{\prime}\right]}$, we have $\operatorname{Ad}_{\left[\int_{r}^{\bullet} \phi\right]^{-1}}(\psi-\phi) \in \mathfrak{D}_{\left[r, r^{\prime}\right]}$, with

$$
\left[\int_{r}^{t} \phi\right]^{-1}\left[\int_{r}^{t} \psi\right]=\int_{r}^{t} \operatorname{Ad}_{\left[\int_{r}^{\bullet} \phi\right]^{-1}}(\psi-\phi) .
$$

c) For $r=t_{0}<\ldots<t_{n}=r^{\prime}$ and $\phi \in \mathfrak{D}_{\left[r, r^{\prime}\right]}$, we have

$$
\int_{r}^{t} \phi=\int_{t_{p}}^{t} \phi \cdot \int_{t_{p-1}}^{t_{p}} \phi \cdot \ldots \cdot \int_{r}^{t_{1}} \phi \quad \forall t \in\left(t_{p}, t_{p+1}\right], p=0, \ldots, n-1 .
$$

d) For $\varrho:\left[\ell, \ell^{\prime}\right] \rightarrow\left[r, r^{\prime}\right]$ of class $C^{1}$ and $\phi \in \mathfrak{D}_{\left[r, r^{\prime}\right]}$, we have $\dot{\varrho} \cdot(\phi \circ \varrho) \in \mathfrak{D}_{\left[\ell, \ell^{\prime}\right]}$, with

$$
\int_{r}^{\varrho} \phi=\left[\int_{\ell}^{\bullet} \dot{\varrho} \cdot(\phi \circ \varrho)\right] \cdot\left[\int_{r}^{\varrho(\ell)} \phi\right] .
$$

e) For each homomorphism $\Psi: G \rightarrow H$ between Lie groups $G$ and $H$ that is of class $C^{1}$, we have

$$
\Psi \circ \int_{r}^{\bullet} \phi=\int_{r}^{\bullet} \mathrm{d}_{e} \Psi \circ \phi \quad \forall \phi \in \mathfrak{D}_{\left[r, r^{\prime}\right]} .
$$

We say that $G$ is $C^{k}$-semiregular for $k \in \mathbb{N} \sqcup\{$ lip, $\infty\}$ if $D_{\mathrm{k}}=C^{k}([0,1], \mathfrak{g})$ holds; which, by d), is equivalent to that $\mathfrak{D}_{\left[r, r^{\prime}\right]}^{k}=C^{k}\left(\left[r, r^{\prime}\right], \mathfrak{g}\right)$ holds for each $\left[r, r^{\prime}\right] \in \mathfrak{K}$, cf. e.g., Lemma 12 in [7]. 


\subsubsection{The Exponential Map}

The exponential map is defined by

$$
\exp : \operatorname{dom}[\exp ] \equiv \mathfrak{i}^{-1}\left(\mathrm{D}_{\mathrm{c}}\right) \rightarrow G,\left.\quad X \mapsto \int \phi_{X}\right|_{[0,1]}=\left(\operatorname{evol}_{\mathrm{c}} \circ \mathfrak{i}\right)(X)
$$

with $\mathfrak{i}:\left.\mathfrak{g} \ni X \rightarrow \phi_{X}\right|_{[0,1]} \in C^{\mathrm{c}}([0,1], \mathfrak{g})$.

Then, instead of saying that $G$ is $C^{\mathrm{C}}$-semiregular, in the following we will rather say that $G$ admits an exponential map. We furthermore remark that $d)$ implies $\mathbb{R} \cdot \operatorname{dom}[\exp ] \subseteq \operatorname{dom}[\exp ]$; and that $t \mapsto \exp (t \cdot X)$ is a 1-parameter group for each $X \in \operatorname{dom}[\exp ]$, with

$$
\exp (t \cdot X)=\left.\left.\int t \cdot \phi_{X}\right|_{[0,1]} \stackrel{d)}{=} \int_{0}^{t} \phi_{X}\right|_{[0,1]} \quad \forall t \geq 0,
$$

cf., e.g., Remark 2.1) in [7]. Finally, if $G$ is abelian, then $X+Y \in \operatorname{dom}[\exp ]$ holds for all $X, Y \in$ $\operatorname{dom}[\exp ]$, because we have

$$
\left.\left.\exp (X) \cdot \exp (Y) \stackrel{a a}{=} \int \phi_{X}\right|_{[0,1]} \cdot \int \phi_{Y}\right|_{[0,1]}=\left.\int \phi_{X+Y}\right|_{[0,1]} .
$$

\subsubsection{Standard Topologies}

We say that $G$ is $\boldsymbol{C}^{\boldsymbol{k}}$-continuous for $k \in \mathbb{N} \sqcup\{$ lip, $\infty, \mathrm{c}\}$ if evol is $_{\mathrm{k}}$ continuous w.r.t. the $C^{k}$-topology. We explicitly remark that under the identification $\mathfrak{i}: \mathfrak{g} \rightarrow\left\{\left.\phi_{X}\right|_{[0,1]} \mid X \in \mathfrak{g}\right\}$, the $C^{\mathrm{c}}$-topology just equals the subspace topology on $\operatorname{dom}[\exp ]$ that is inherited by the locally convex topology on $\mathfrak{g}$. So, instead of saying that $G$ is $C^{\mathrm{c}}$-continuous if $\mathrm{evol}_{\mathrm{c}}$ is continuous w.r.t. this topology, we will rather say that the exponential map is continuous.

\subsection{The Riemann Integral}

Let $F$ be a Hausdorff locally convex vector space with system of continuous seminorms $\mathfrak{Q}$, and completion $\bar{F}$. For each $\mathfrak{q} \in \mathfrak{P}$, we let $\overline{\mathfrak{q}}: \bar{F} \rightarrow \mathbb{R}_{\geq 0}$ denote the continuous extension of $\mathfrak{q}$ to $\bar{F}$. The Riemann integral of $\gamma \in C^{0}\left(\left[r, r^{\prime}\right], F\right)$ (for $\left.\left[r, r^{\prime}\right] \in \mathfrak{K}\right)$ is denoted by $\int \gamma(s) \mathrm{d} s \in \bar{F}$; and we define

$$
\int_{a}^{b} \gamma(s) \mathrm{d} s:=\left.\int \gamma\right|_{[a, b]}(s) \mathrm{d} s, \quad \int_{b}^{a} \gamma(s) \mathrm{d} s:=-\int_{a}^{b} \gamma(s) \mathrm{d} s, \quad \int_{c}^{c} \gamma(s) \mathrm{d} s:=0
$$

for $r \leq a<b \leq r^{\prime}$ and $c \in\left[r, r^{\prime}\right]$. Clearly, the Riemann integral is linear, with

$$
\begin{aligned}
\int_{a}^{c} \gamma(s) \mathrm{d} s & =\int_{a}^{b} \gamma(s) \mathrm{d} s+\int_{b}^{c} \gamma(s) \mathrm{d} s & & \forall r \leq a<b<c \leq r^{\prime} \\
\gamma-\gamma(r) & =\int_{r}^{\bullet} \dot{\gamma}(s) \mathrm{d} s & & \forall \gamma \in C^{1}\left(\left[r, r^{\prime}\right], F\right), \\
\mathfrak{q}(\gamma-\gamma(r)) & \leq \int_{r}^{\bullet} \mathfrak{q}(\dot{\gamma}(s)) \mathrm{d} s & & \forall \mathfrak{q} \in \mathfrak{Q}, \gamma \in C^{1}\left(\left[r, r^{\prime}\right], F\right),
\end{aligned}
$$

as well as

$$
\overline{\mathfrak{q}}\left(\int_{r}^{\bullet} \gamma(s) \mathrm{d} s\right) \leq \int_{r}^{\bullet} \mathfrak{q}(\gamma(s)) \mathrm{d} s \quad \forall \mathfrak{q} \in \mathfrak{Q}, \gamma \in C^{0}\left(\left[r, r^{\prime}\right], F\right)
$$

We furthermore have the substitution formula

$$
\int_{r}^{\varrho(t)} \gamma(s) \mathrm{d} s=\int_{\ell}^{t} \dot{\varrho}(s) \cdot(\gamma \circ \varrho)(s) \mathrm{d} s
$$

for each $\gamma \in C^{0}\left(\left[r, r^{\prime}\right], F\right)$, and each $\varrho: \mathfrak{K} \ni\left[\ell, \ell^{\prime}\right] \rightarrow\left[r, r^{\prime}\right]$ of class $C^{1}$ with $\varrho(\ell)=r$ and $\varrho\left(\ell^{\prime}\right)=r^{\prime}$. Moreover, if $E$ is a Hausdorff locally convex vector space, and $\mathfrak{L}: F \rightarrow E$ is a continuous linear map, then we have

$$
\int \gamma(s) \mathrm{d} s \in F \quad \text { for } \quad \gamma \in C^{0}\left(\left[r, r^{\prime}\right], F\right) \quad \Longrightarrow \quad \mathfrak{L}\left(\int \gamma(s) \mathrm{d} s\right)=\int \mathfrak{L}(\gamma(s)) \mathrm{d} s .
$$


Finally, for $\gamma \in \mathrm{CP}^{0}\left(\left[r, r^{\prime}\right], F\right)$ with $\gamma[0], \ldots, \gamma[n-1]$ as in (2) , we define

$$
\int \gamma(s) \mathrm{d} s:=\sum_{p=0}^{n-1} \int \gamma[p](s) \mathrm{d} s .
$$

A standard refinement argument in combination with (6) then shows that this is well defined; i.e., independent of any choices we have made. We define $\int_{a}^{b} \gamma(s) \mathrm{d} s, \int_{b}^{a} \gamma(s) \mathrm{d} s$ and $\int_{c}^{c} \gamma(s) \mathrm{d} s$ as in (5); and observe that (12) is linear and fulfills (6).

\subsection{Standard Facts and Estimates}

Let $F_{1}, \ldots, F_{n}, E$ be Hausdorff locally convex vector spaces with corresponding system of continuous seminorms $\mathfrak{Q}_{1}, \ldots, \mathfrak{Q}_{n}, \mathfrak{P}$. We recall that

Lemma 2. Let $X$ be a topological space; and let $\Phi: X \times F_{1} \times \ldots \times F_{n} \rightarrow E$ be continuous with $\Phi(x, \cdot)$ n-multilinear for each $x \in X$. Then, for each compact $\mathrm{K} \subseteq X$ and each $\mathfrak{p} \in \mathfrak{P}$, there exist seminorms $\mathfrak{q}_{1} \in \mathfrak{Q}_{1}, \ldots, \mathfrak{q}_{n} \in \mathfrak{Q}_{n}$ as well as $O \subseteq X$ open with $\mathrm{K} \subseteq O$, such that

$$
(\mathfrak{p} \circ \Phi)\left(y, X_{1}, \ldots, X_{n}\right) \leq \mathfrak{q}_{1}\left(X_{1}\right) \cdot \ldots \cdot \mathfrak{q}_{n}\left(X_{n}\right) \quad \forall y \in O
$$

holds for all $X_{1} \in F_{1}, \ldots, X_{n} \in F_{n}$.

Proof. Confer, e.g., Corollary 1 in [7].

Next, given Hausdorff locally convex vector spaces $F_{1}, F_{2}$, and a continuous linear map $\Phi: F_{1} \rightarrow F_{2}$, we denote its unique continuous linear extension by $\bar{\Phi}: \bar{F}_{1} \rightarrow \bar{F}_{2}$ (cf., 2. Theorem in Sect. 3.4 in [10]). We recall that

Lemma 3. Let $F_{1}, F_{2}$ be Hausdorff locally convex vector spaces; and let $f: F_{1} \supseteq U \rightarrow F_{2}$ be of class $C^{2}$. Suppose that $\gamma: D \rightarrow F_{1} \subseteq \bar{F}$ is continuous at $t \in D$, such that $\lim _{h \rightarrow 0} 1 / h \cdot(\gamma(t+h)-\gamma(t))=$ : $X \in \bar{F}_{1}$ exists. Then, we have

$$
\lim _{h \rightarrow 0} 1 / h \cdot(f(\gamma(t+h))-f(\gamma(t)))=\overline{\mathrm{d}_{\gamma(t)} f}(X) .
$$

Proof. Confer, e.g., Lemma 7 in [7].

Remark 3. Let $F$ be a Hausdorff locally convex vector space, let $U \subseteq F$ be open, and let $G$ be a Lie group. A map $f: U \rightarrow G$ is said to be

- differentiable at $x \in U$ if there exists a chart $\left(\Xi^{\prime}, \mathcal{U}^{\prime}\right)$ of $G$ with $f(x) \in \mathfrak{U}^{\prime}$, such that

$$
\left(D_{v}^{\Xi^{\prime}} f\right)(x):=\lim _{h \rightarrow 0} 1 / h \cdot\left(\left(\Xi^{\prime} \circ f\right)(x+h \cdot v)-\left(\Xi^{\prime} \circ f\right)(x)\right) \in E \quad \forall v \in F
$$

exists. Then, Lemma 3 applied to coordinate changes shows that (13) holds for one chart around $f(x)$ if and only if it holds for each chart around $f(x)$ - and that

$$
\mathrm{d}_{x} f(v):=\left(\mathrm{d}_{\Xi^{\prime}(f(x))} \Xi^{\prime-1} \circ\left(D_{v}^{\Xi^{\prime}} f\right)\right)(x) \in T_{f(x)} G \quad \forall v \in F
$$

is independent of the explicit choice of $\left(\Xi^{\prime}, U^{\prime}\right)$.

- differentiable if $f$ is differentiable at each $x \in U$.

In particular, Lemma 2 provides us with the following statements (cf. also Sect. 3.4.1 in [7]):

I) Since $\operatorname{Ad}: G \times \mathfrak{g} \ni(g, X) \mapsto \operatorname{Ad}_{g}(X) \in \mathfrak{g}$ is smooth as well as linear in the second argument (by Lemma 2), to each compact $\mathrm{C} \subseteq G$ and each $\mathfrak{v} \in \mathfrak{P}$, there exists some $\mathfrak{v} \leq \mathfrak{w} \in \mathfrak{P}$, such that $\mathfrak{v} \circ \operatorname{Ad}_{g} \leq \mathfrak{w}$ holds for each $g \in \mathrm{C}$. 
II) By Lemma 2 applied to $\Phi \equiv$ Ad and $K \equiv\{e\}$, to each $\mathfrak{m} \in \mathfrak{P}$, there exists some $\mathfrak{m} \leq \mathfrak{q} \in \mathfrak{P}$, as well as $O \subseteq G$ symmetric open with $e \in O$, such that $\mathfrak{m} \circ \operatorname{Ad}_{g} \leq \mathfrak{q q}$ holds for each $g \in O$.

III) Suppose that $\operatorname{im}[\mu] \subseteq \mathcal{U}$ holds for $\mu \in C^{1}\left(\left[r, r^{\prime}\right], G\right)$. Then, we have

$$
\delta^{r}(\mu)=\omega\left(\Xi \circ \mu, \partial_{t}(\Xi \circ \mu)\right)
$$

for the smooth map $\omega: \mathcal{V} \times E \ni(x, X) \mapsto \mathrm{d}_{\Xi^{-1}(x)} \mathrm{R}_{\left[\Xi^{-1}(x)\right]^{-1}}\left(\mathrm{~d}_{x} \Xi^{-1}(X)\right) \in \mathfrak{g}$. Since $\omega$ is linear in the second argument, (by Lemma 2) for each $\mathfrak{q} \in \mathfrak{P}$, there exists some $\mathfrak{q} \leq \mathfrak{m} \in \mathfrak{P}$ with

$$
(. \mathfrak{q} \circ \omega)(x, X) \leq \mathfrak{m}(X) \quad \forall x \in \overline{\mathrm{B}}_{\mathfrak{m}, 1}, X \in E .
$$

IV) Suppose that $\operatorname{im}[\mu] \subseteq \mathcal{U}$ holds for $\mu \in C^{1}\left(\left[r, r^{\prime}\right], G\right)$. Then, we have

$$
\partial_{t}(\Xi \circ \mu)=v\left(\Xi \circ \mu, \delta^{r}(\mu)\right)
$$

for the smooth map $v: \mathcal{V} \times \mathfrak{g} \ni(x, X) \mapsto\left(\mathrm{d}_{\Xi^{-1}(x)} \Xi \circ \mathrm{d}_{e} \mathrm{R}_{\Xi^{-1}(x)}\right)(X) \in E$. Since $v$ is linear in the second argument, (by Lemma 2) for each $\mathfrak{q} \in \mathfrak{P}$, there exists some $\mathfrak{u} \leq \mathfrak{m} \in \mathfrak{P}$ with

$$
(\mathfrak{u} \circ v)(x, X) \leq \mathfrak{m}(X) \quad \forall x \in \overline{\mathrm{B}}_{\mathfrak{m}, 1}, \quad X \in \mathfrak{g} .
$$

For each $\mu \in C^{1}\left(\left[r, r^{\prime}\right], G\right)$ with $\operatorname{im}[\Xi \circ \mu] \subseteq \overline{\mathrm{B}}_{\mathfrak{m}, 1}$, we thus obtain from (16), (7), and (8) that

$$
\mathfrak{u}(\Xi \circ \mu)=\mathfrak{u}\left(\int_{r}^{\bullet} v\left((\Xi \circ \mu)(s), \delta^{r}(\mu)(s)\right) \mathrm{d} s\right) \leq \int_{r}^{\bullet} \cdot \mathfrak{m}\left(\delta^{r}(\mu)(s)\right) \mathrm{d} s .
$$

For instance, we immediately obtain from (17) that

Lemma 4. For each $\mathfrak{u} \in \mathfrak{P}$, there exist $\mathfrak{u} \leq \mathfrak{m} \in \mathfrak{P}$, and $U \subseteq G$ open with $e \in U$, such that

$$
(\mathfrak{u} \circ \Xi)\left(\int_{r}^{\bullet} \chi\right) \leq \int_{r}^{\bullet} \mathfrak{m}(\chi(s)) \mathrm{d} s
$$

holds, for each $\chi \in \mathfrak{D}_{\left[r, r^{\prime}\right]}$ with $\int_{r}^{\bullet} \chi \in U$; for all $\left[r, r^{\prime}\right] \in \mathfrak{K}$.

Moreover,

Lemma 5. We have $\operatorname{Ad}_{\mu}(\phi) \in C^{k}\left(\left[r, r^{\prime}\right], \mathfrak{g}\right)$ for each $\mu \in C^{k+1}\left(\left[r, r^{\prime}\right], G\right), \phi \in C^{k}\left(\left[r, r^{\prime}\right], \mathfrak{g}\right)$, and $k \in \mathbb{N} \sqcup\{$ lip, $\infty\}$.

Proof. Confer, e.g., Lemma 13 in [7].

Lemma 6. Let $\left[r, r^{\prime}\right] \in \mathfrak{K}, k \in \mathbb{N} \sqcup\{\infty\}$, and $\phi \in \mathfrak{D}_{\left[r, r^{\prime}\right]}^{k}$ be fixed. Then, for each $\mathfrak{p} \in \mathfrak{P}$ and $\mathrm{s} \preceq k$, there exists some $\mathfrak{p} \leq \mathfrak{q} \in \mathfrak{P}$ with

$$
\cdot \mathfrak{p}_{\infty}^{\mathrm{p}}\left(\operatorname{Ad}_{\left[\int_{r}^{\bullet} \phi\right]^{-1}}(\psi)\right) \leq \cdot \mathfrak{q}_{\infty}^{\mathrm{p}}(\psi) \quad \forall \psi \in C^{k}\left(\left[r, r^{\prime}\right], \mathfrak{g}\right), \quad 0 \leq \mathrm{p} \leq \mathrm{s} .
$$

Proof. Confer, e.g., Lemma 14 in [7].

Then, modifying the argumentation used in the proof of the Lipschitz case in Lemma 13 in [7] to our deviating convention concerning the topology on the set of Lipschitz curves, we also obtain

Lemma 7. Let $\left[r, r^{\prime}\right] \in \mathfrak{K}$, and $\phi \in \mathfrak{D}_{\left[r, r^{\prime}\right]}$ be fixed. Then, for each $\mathfrak{p} \in \mathfrak{P}$, there exists some $\mathfrak{p} \leq \mathfrak{q} \in \mathfrak{P}$ with

$$
\mathfrak{p}_{\infty}^{\operatorname{lip}}\left(\operatorname{Ad}_{\left[\int_{r}^{\bullet} \phi\right]^{-1}}(\psi)\right) \leq \cdot \mathfrak{q}_{\infty}^{\operatorname{lip}}(\psi) \quad \forall \psi \in C^{\operatorname{lip}}\left(\left[r, r^{\prime}\right], \mathfrak{g}\right) .
$$

Proof. Confer Appendix A.2. 


\subsection{Continuity Statements}

For $h \in G$, we define $\Xi_{h}(g):=\Xi\left(h^{-1} \cdot g\right)$ for each $g \in h \cdot \mathcal{U}$; and recall that, cf. Lemma 8 in [7]

Lemma 8. Let $\mathrm{C} \subseteq \mathcal{U}$ be compact. Then, for each $\mathfrak{p} \in \mathfrak{P}$, there exists some $\mathfrak{p} \leq \mathfrak{u} \in \mathfrak{P}$, and a symmetric open neighbourhood $V \subseteq \mathcal{U}$ of e with $\mathrm{C} \cdot V \subseteq \mathcal{U}$ and $\overline{\mathrm{B}}_{\mathfrak{u}, 1} \subseteq \Xi(V)$, such that

$$
\mathfrak{p}\left(\Xi(q)-\Xi\left(q^{\prime}\right)\right) \leq \mathfrak{u}\left(\Xi_{g \cdot h}(q)-\Xi_{g \cdot h}\left(q^{\prime}\right)\right) \quad \forall q, q^{\prime} \in g \cdot V, h \in V
$$

holds for each $g \in \mathrm{C}$.

Now, combining Lemma 4 with Lemma 8, we obtain the following variation of Proposition 1 in [7]:

Lemma 9. For each $\mathfrak{p} \in \mathfrak{P}$, there exist $\mathfrak{p} \leq \mathfrak{q} \in \mathfrak{P}$ and $V \subseteq G$ open with $e \in V$, such that

$$
\mathfrak{p}\left(\Xi\left(\int_{r}^{\bullet} \phi\right)-\Xi\left(\int_{r}^{\bullet} \psi\right)\right) \leq \int_{r}^{\bullet} \mathfrak{q}(\phi(s)-\psi(s)) \mathrm{d} s
$$

holds for all $\phi, \psi \in \mathfrak{D}_{\left[r, r^{\prime}\right]}$ with $\int_{r}^{\bullet} \phi, \int_{r}^{\bullet} \psi \in V ;$ for each $\left[r, r^{\prime}\right] \in \mathfrak{K}$.

Proof. We let $\mathfrak{p} \leq \mathfrak{u} \in \mathfrak{P}$ and $V$ be as in Lemma 8 for $\mathrm{C} \equiv\{e\}$ there (i.e., $V$ is symmetric with $\left.\overline{\mathrm{B}}_{\mathfrak{u}, 1} \subseteq \Xi(V)\right)$. We choose $U \subseteq G$ and $\mathfrak{u} \leq \mathfrak{m} \in \mathfrak{P}$ as in Lemma4. We furthermore let $\mathfrak{m} \leq \mathfrak{q} \in \mathfrak{P}$ and $O \subseteq G$ be as in II). Then, shrinking $V$ if necessary, we can assume that $V^{-1} \cdot V \subseteq U$ as well as $V \subseteq O$ holds. Then, for $\phi, \psi$ as in the presumptions, Lemma 8 applied to $q \equiv \int_{r}^{\bullet} \phi, q^{\prime} \equiv \int_{r}^{\bullet} \psi, h \equiv \int_{r}^{\bullet} \phi \in V$, and $g \equiv e$ gives

$$
\mathfrak{p}\left(\Xi\left(\int_{r}^{\bullet} \phi\right)-\Xi\left(\int_{r}^{\bullet} \psi\right)\right) \leq \mathfrak{u}\left(\Xi_{\int_{r}^{\bullet} \phi}\left(\int_{r}^{\bullet} \phi\right)-\Xi_{\int_{r}^{\bullet} \phi}\left(\int_{r}^{\bullet} \psi\right)\right)=(\mathfrak{u} \circ \Xi)\left(\left[\int_{r}^{\bullet} \phi\right]^{-1}\left[\int_{r}^{\bullet} \psi\right]\right) .
$$

By assumption, for each $t \in\left[r, r^{\prime}\right]$, we have

$$
U \supseteq V^{-1} \cdot V \ni\left[\int_{r}^{t} \phi\right]^{-1}\left[\int_{r}^{t} \psi\right] \stackrel{b]}{=} \int_{r}^{t} \operatorname{Ad}_{\left[\int_{r}^{\bullet} \phi\right]^{-1}}(\psi-\phi) \quad \text { with } \quad\left[\int_{r}^{\bullet} \phi\right]^{-1} \in V^{-1}=V \subseteq O .
$$

We obtain from Lemma 4 and II) that

$$
(\mathfrak{u} \circ \Xi)\left(\left[\int_{r}^{t} \phi\right]^{-1}\left[\int_{r}^{t} \psi\right]\right) \leq \int_{r}^{t} \cdot \mathfrak{m}\left(\operatorname{Ad}_{\left[\int_{r}^{s} \phi\right]^{-1}}(\psi(s)-\phi(s))\right) \mathrm{d} s \leq \int_{r}^{t} \cdot \mathfrak{q}(\psi(s)-\phi(s)) \mathrm{d} s
$$

holds for each $t \in\left[r, r^{\prime}\right]$; which proves the claim.

We furthermore observe that

Lemma 10. Suppose that $\exp : \operatorname{dom}[\exp ] \rightarrow G$ is continuous; and let $X \in \operatorname{dom}[\exp ]$ be fixed. Then, for each open neighbourhood $V \subseteq G$ of e, there exists some $\mathfrak{m} \in \mathfrak{P}$, such that

$$
\mathfrak{m}(Y-X) \leq 1 \quad \text { for }\left.\left.\quad Y \in \operatorname{dom}[\exp ] \quad \Longrightarrow \quad \int_{0}^{\bullet} \phi_{Y}\right|_{[0,1]} \in \int_{0}^{\bullet} \phi_{X}\right|_{[0,1]} \cdot V .
$$

Proof. By assumption, $\alpha$ : $[0,1] \times \operatorname{dom}[\exp ] \ni(t, Y) \mapsto \exp (t \cdot X)^{-1} \cdot \exp (t \cdot Y)$ is continuous; and we have $\alpha(\cdot, X)=e$. For $\tau \in[0,1]$ fixed, there thus exists an open interval $I_{\tau} \subseteq \mathbb{R}$ containing $\tau$, as well as an open neighbourhood $O_{\tau} \subseteq \mathfrak{g}$ of $X$, such that we have

$$
\exp (t \cdot X)^{-1} \cdot \exp (t \cdot Y) \in V \quad \forall t \in I_{\tau} \cap[0,1], \quad Y \in O_{\tau} \cap \operatorname{dom}[\exp ] .
$$

We choose $\tau_{1}, \ldots, \tau_{n} \in[0,1]$ with $[0,1] \subseteq I_{\tau_{1}} \cup \ldots \cup I_{\tau_{n}}$; and define $O:=O_{\tau_{1}} \cap \ldots \cap O_{\tau_{n}}$. Then, (18) holds for each $t \in[0,1]$ and $Y \in O \cap \operatorname{dom}[\exp ]$; so that the claim holds for each fixed $\mathfrak{m} \in \mathfrak{P}$ with $\overline{\mathrm{B}}_{\mathfrak{m}, 1} \subseteq O$.

\section{Auxiliary Results}

In this section, we introduce the continuity notions that we will need to formulate our main results. We furthermore provide some elementary continuity statements that we will need in the main text. 


\subsection{Sets of Curves}

Let $\left[r, r^{\prime}\right] \in \mathfrak{K}$ be fixed. We will tacitly use in the following that $C^{k}\left(\left[r, r^{\prime}\right], \mathfrak{g}\right)$ is a real vector space for each $k \in \mathbb{N} \sqcup\{$ lip, $\infty, \mathrm{c}\}$. We will furthermore use that:

A) For each $k \in \mathbb{N} \sqcup\{\operatorname{lip}, \infty\}, \phi \in \mathfrak{D}_{\left[r, r^{\prime}\right]}^{k}$, and $\psi \in C^{k}\left(\left[r, r^{\prime}\right], \mathfrak{g}\right)$, we have $\operatorname{Ad}_{\left[\int_{r}^{\bullet} \phi\right]^{-1}}(\psi) \in C^{k}\left(\left[r, r^{\prime}\right], \mathfrak{g}\right)$ by Lemma 5. Evidently, the same statement also holds for $k=\mathrm{c}$ if $G$ is abelian.

B) For each $k \in \mathbb{N} \sqcup\{\operatorname{lip}, \infty, c\}, \phi \in \mathfrak{D}_{\left[r, r^{\prime}\right]}^{k},\left[\ell, \ell^{\prime}\right] \in \mathfrak{K}$, and

$$
\varrho:\left[\ell, \ell^{\prime}\right] \rightarrow\left[r, r^{\prime}\right], \quad t \mapsto r+(t-\ell) \cdot\left(r^{\prime}-r\right) /\left(\ell^{\prime}-\ell\right),
$$

we have $\dot{\varrho} \cdot(\phi \circ \varrho)=\left(r^{\prime}-r\right) /\left(\ell^{\prime}-\ell\right) \cdot(\phi \circ \varrho) \in \mathfrak{D}_{\left[\ell, \ell^{\prime}\right]}^{k}$ by $\left.d\right)$, with

$$
\begin{aligned}
\cdot \mathfrak{p}_{\infty}^{\mathrm{s}}(\dot{\varrho} \cdot(\phi \circ \varrho)) & =\left[\frac{\left(r^{\prime}-r\right)}{\left(\ell^{\prime}-\ell\right)}\right]^{\mathrm{s}+1} \cdot \mathfrak{p}_{\infty}^{\mathrm{s}}(\phi) \quad \text { with } \quad \mathrm{s} \preceq k \quad \text { for } \quad k \in \mathbb{N} \sqcup\{\infty, c\}, \\
\operatorname{Lip}(\cdot \mathfrak{p}, \dot{\varrho} \cdot(\phi \circ \varrho)) & =\left[\frac{\left(r^{\prime}-r\right)}{\left(\ell^{\prime}-\ell\right)}\right]^{2} \cdot \operatorname{Lip}(\cdot \mathfrak{p}, \phi) \quad \text { for } \quad k=\operatorname{lip} .
\end{aligned}
$$

We say that $\mathfrak{g}$ is $\mathbf{k}$-complete for $k \in \mathbb{N} \sqcup\{$ lip, $\infty, \mathrm{c}\}$ if

$$
\int \operatorname{Ad}_{\left[\int_{r}^{s} \phi\right]^{-1}}(\chi(s)) \mathrm{d} s \in \mathfrak{g}
$$

holds for all $\phi, \chi \in \mathfrak{D}_{\left[r, r^{\prime}\right]}^{k}$, for each $\left[r, r^{\prime}\right] \in \mathfrak{K}$. Then,

\section{Remark 4.}

- $\mathfrak{g}$ is c-complete if $G$ is abelian.

- $\mathfrak{g}$ is $\mathrm{k}$-complete for $k \in \mathbb{N} \sqcup\{$ lip, $\infty, \mathrm{c}\}$ if and only if (19) holds for $\left[r, r^{\prime}\right] \equiv[0,1]$.

For this, let $\phi, \chi \in \mathfrak{D}_{\left[r, r^{\prime}\right]}^{k}$ be given. Then, for $\varrho:[0,1] \rightarrow\left[r, r^{\prime}\right]$ as in $\left.\left.B\right)\right]$ with $\left[\ell, \ell^{\prime}\right] \equiv[0,1]$ there, we have $\dot{\varrho} \cdot(\phi \circ \varrho), \dot{\varrho} \cdot(\chi \circ \varrho) \in \mathfrak{D}_{[0,1]}^{k}$ with

$$
\begin{aligned}
\int \operatorname{Ad}_{\left[\int_{r}^{s} \phi\right]^{-1}}(\chi(s)) \mathrm{d} s & =\int_{r}^{\varrho(1)} \operatorname{Ad}_{\left[\int_{r}^{s} \phi\right]^{-1}}(\chi(s)) \mathrm{d} s \\
& \stackrel{(10)}{=} \int_{0}^{1} \dot{\varrho}(s) \cdot \operatorname{Ad}_{\left[\int_{r}^{\varrho(s)} \phi\right]^{-1}}(\chi(\varrho(s))) \mathrm{d} s \\
& =\int_{0}^{1} \operatorname{Ad}_{\left[\int_{r}^{\varrho(s)} \phi\right]^{-1}}((\varrho \cdot(\chi \circ \varrho))(s)) \mathrm{d} s \\
& \underline{d)} \int_{0}^{1} \operatorname{Ad}_{\left[\int_{0}^{s} \dot{\varrho} \cdot(\phi \circ \varrho)\right]^{-1}}((\dot{\varrho} \cdot(\chi \circ \varrho))(s)) \mathrm{d} s .
\end{aligned}
$$

In particular, Point $A$ ) then shows:

- If $G$ is $C^{0}$-semiregular, then $\mathfrak{g}$ is 0-complete if and only if $\mathfrak{g}$ is integral complete - i.e., if and only if $\int \phi(s) \mathrm{d} s \in \mathfrak{g}$ holds for each $\phi \in C^{0}([0,1], \mathfrak{g})$.

- If $G$ is $C^{k}$-semiregular for $k \in \mathbb{N}_{\geq 1} \sqcup\{$ lip, $\infty\}$, then $\mathfrak{g}$ is $\mathrm{k}$-complete if and only if $\mathfrak{g}$ is Mackeycomplete 8

\footnotetext{
${ }_{8}^{8}$ Recall that $\mathfrak{g}$ is Mackey complete if and only if $\int \phi(s) \mathrm{d} s \in \mathfrak{g}$ holds for each $\phi \in C^{k}([0,1], \mathfrak{g})$, for any $k \in \mathbb{N} \geq 1 \sqcup\{\operatorname{lip}, \infty\}$, cf., 2.14 Theorem in 12 .
} 


\subsection{Weak Continuity}

A pair $(\phi, \psi) \in C^{0}\left(\left[r, r^{\prime}\right], \mathfrak{g}\right) \times C^{0}\left(\left[r, r^{\prime}\right], \mathfrak{g}\right)$ is said to be

- admissible if $\phi+(-\delta, \delta) \cdot \psi \subseteq \mathfrak{D}_{\left[r, r^{\prime}\right]}$ holds for some $\delta>0$.

- regular if it is admissible with

$$
\lim _{h \rightarrow 0}^{\infty} \int_{r}^{\bullet} \phi+h \cdot \psi=\int_{r}^{\bullet} \phi .
$$

Then,

\section{Remark 5.}

1) It follows from c) that $(\phi, \chi)$ is admissible/regular if and only if $\left(\left.\phi\right|_{\left[\ell, \ell^{\prime}\right]},\left.\chi\right|_{\left[\ell, \ell^{\prime}\right]}\right)$ is admissible/regular for each $r \leq \ell<\ell^{\prime} \leq r^{\prime}$.

2) Each $(0, \mathrm{i}(X))$ with $X \in \operatorname{dom}[\exp ]$ is regular; because we have

$$
\left.\left.\left.\int_{0}^{t} h \cdot \phi_{X}\right|_{[0,1]} \stackrel{(4,)}{=} \int t h \cdot \phi_{X}\right|_{[0,1]} \stackrel{(\underline{4})}{=} \int_{0}^{t h} \phi_{X}\right|_{[0,1]}
$$

for each $t \in[0,1]$, and each $h \in \mathbb{R}$.

We say that $G$ is weakly k-continuous for $k \in \mathbb{N} \sqcup\{$ lip, $\infty, c\}$ if each admissible $(\phi, \psi) \in$ $C^{k}([0,1], \mathfrak{g}) \times C^{k}([0,1], \mathfrak{g})$ is regular.

Lemma 11. If $G$ is weakly $\mathrm{k}$-continuous for $k \in \mathbb{N} \sqcup\{$ lip, $\infty, \mathrm{c}\}$, then each admissible $(\phi, \psi) \in$ $C^{k}\left(\left[r, r^{\prime}\right], \mathfrak{g}\right) \times C^{k}\left(\left[r, r^{\prime}\right], \mathfrak{g}\right)$ (for each $\left.\left[r, r^{\prime}\right] \in \mathfrak{K}\right)$ is regular.

Proof. We define $\varrho:[0,1] \ni t \mapsto r+t \cdot\left(r^{\prime}-r\right) \in\left[r, r^{\prime}\right]$; and observe that

$$
\begin{gathered}
\int_{r}^{\varrho} \phi \stackrel{d)}{=} \int_{0}^{\bullet} \dot{\varrho} \cdot(\phi \circ \varrho), \\
\int_{r}^{\varrho}[\phi+h \cdot \psi] \stackrel{d)]}{=} \int_{0}^{\bullet}[\underline{\varrho} \cdot(\phi \circ \varrho)+h \cdot \dot{\varrho} \cdot(\psi \circ \varrho)]
\end{gathered}
$$

holds for $h>0$ suitably small. Since we have $\varrho \cdot(\phi \circ \varrho), \dot{\varrho} \cdot(\psi \circ \varrho) \in C^{k}([0,1], \mathfrak{g})$ by Point B), the claim is clear from the presumptions.

Lemma 12. $G$ is weakly $\mathrm{k}$-continuous for $k \in \mathbb{N} \sqcup\{$ lip, $\infty\}$ if and only if

$$
\lim _{h \rightarrow 0}^{\infty} \int_{0}^{\bullet} h \cdot \chi=e
$$

holds, for each $\chi \in \mathrm{D}_{\mathrm{k}}$ with $(-\delta, \delta) \cdot \chi \subseteq \mathrm{D}_{\mathrm{k}}$ for some $\delta>0$. The same statement also holds for $k=\mathrm{c}$ if $G$ is abelian.

Proof. The one implication is evident. For the other implication, we suppose that $(\phi, \psi) \in$ $C^{k}([0,1], \mathfrak{g}) \times C^{k}([0,1], \mathfrak{g})$ is admissible. Since $\phi \in \mathfrak{D}_{[0,1]}^{k}$ holds, we have

$$
\left[\int_{0}^{t} \phi\right]^{-1}\left[\int_{0}^{t} \phi+h \cdot \psi\right] \stackrel{b)}{=} \int_{0}^{t} h \cdot \operatorname{Ad}_{\left[\int_{0}^{\bullet} \phi\right]^{-1}}(\psi) \quad \forall t \in[0,1]
$$

with $\chi:=\operatorname{Ad}_{\left[\int_{0}^{\bullet} \phi\right]^{-1}}(\psi) \in C^{k}([0,1], \mathfrak{g})$ by Point $\left.\mathrm{A}\right)$, The claim is thus clear from (20).

Corollary 1. If $G$ is abelian, then $G$ is weakly c-continuous.

Proof. This is clear from Lemma 12 and Remark $[52$ ). 


\subsection{Mackey Continuity}

We write $\left\{\phi_{n}\right\}_{n \in \mathbb{N}} \rightarrow_{\text {m.k }} \phi$ for $k \in \mathbb{N} \sqcup\{\operatorname{lip}, \infty, \mathrm{c}\},\left\{\phi_{n}\right\}_{n \in \mathbb{N}} \subseteq C^{k}\left(\left[r, r^{\prime}\right], \mathfrak{g}\right)$, and $\phi \in C^{k}\left(\left[r, r^{\prime}\right], \mathfrak{g}\right)$ if

$$
\mathfrak{p}_{\infty}^{\mathrm{s}}\left(\phi-\phi_{n}\right) \leq \mathfrak{c}_{\mathfrak{p}}^{\mathrm{s}} \cdot \lambda_{n} \quad \forall n \geq \mathfrak{l}_{\mathfrak{p}}^{\mathrm{s}}, \mathfrak{p} \in \mathfrak{P}, \mathrm{s} \preceq k
$$

holds for certain $\left\{\mathfrak{c}_{\mathfrak{p}}^{\mathrm{s}}\right\}_{\mathfrak{s} \preceq k, \mathfrak{p} \in \mathfrak{P}} \subseteq \mathbb{R}_{\geq 0},\left\{\mathfrak{l}_{\mathfrak{p}}^{\mathrm{s}}\right\}_{\mathfrak{s} \preceq k, \mathfrak{p} \in \mathfrak{P}} \subseteq \mathbb{N}$, and $\mathbb{R}_{\geq 0} \supseteq\left\{\lambda_{n}\right\}_{n \in \mathbb{N}} \rightarrow 0$.

Remark 6. Suppose that $\mathfrak{D}_{\left[r, r^{\prime}\right]}^{k} \supseteq\left\{\phi_{n}\right\}_{n \in \mathbb{N}} \rightarrow_{\mathfrak{m} . \mathrm{k}} \phi \in \mathfrak{D}_{\left[r, r^{\prime}\right]}^{k}$ holds for $k \in \mathbb{N} \sqcup\{$ lip, $\infty, \mathrm{c}\}$. Then,

$$
\left\{\phi_{\iota(n)}\right\}_{n \in \mathbb{N}} \rightarrow_{\mathfrak{m} . \mathrm{k}} \phi
$$

holds for each strictly increasing $\iota: \mathbb{N} \rightarrow \mathbb{N}$.

We say that $G$ is Mackey k-continuous if

$$
\mathrm{D}_{k} \supseteq\left\{\phi_{n}\right\}_{n \in \mathbb{N}} \rightarrow_{\mathfrak{m} . \mathrm{k}} \phi \in \mathrm{D}_{k} \quad \Longrightarrow \quad \lim _{n}^{\infty} \int_{r}^{\bullet} \phi_{n}=\int_{r}^{\bullet} \phi .
$$

In analogy to Lemma 11, we obtain

Lemma 13. $G$ is Mackey k-continuous for $k \in \mathbb{N} \sqcup\{$ lip, $\infty$, c $\}$ if and only if

$$
\mathfrak{D}_{\left[r, r^{\prime}\right]}^{k} \supseteq\left\{\phi_{n}\right\}_{n \in \mathbb{N}} \rightarrow_{\mathfrak{m} . \mathrm{k}} \phi \in \mathfrak{D}_{\left[r, r^{\prime}\right]}^{k} \quad \Longrightarrow \quad \lim _{n}^{\infty} \int_{r}^{\bullet} \phi_{n}=\int_{r}^{\bullet} \phi,
$$

for each $\left[r, r^{\prime}\right] \in \mathfrak{K}$.

Proof. The one implication is evident. For the other implication, we suppose that (22) holds. Then, for $\left[r, r^{\prime}\right] \in \mathfrak{K}$ fixed, we let $\varrho:[0,1] \ni t \mapsto r+t \cdot\left(r^{\prime}-r\right) \in\left[r, r^{\prime}\right]$; and obtain

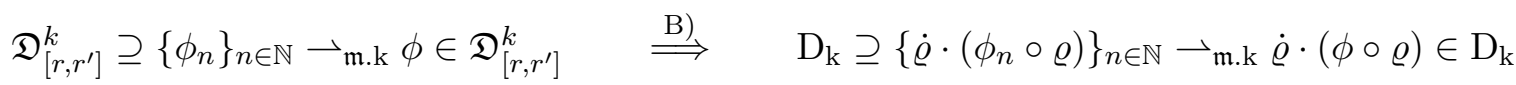

$$
\begin{aligned}
& \Longrightarrow \quad \lim _{n}^{\infty} \int_{r}^{\bullet} \dot{\varrho} \cdot\left(\phi_{n} \circ \varrho\right)=\int_{r}^{\bullet} \dot{\varrho} \cdot(\phi \circ \varrho) \\
& \stackrel{d d)}{\Longrightarrow} \quad \lim _{n}^{\infty} \int_{r}^{\bullet} \phi_{n}=\int_{r}^{\bullet} \phi \text {, }
\end{aligned}
$$

whereby the second step is due to the presumptions.

In analogy to Lemma 12, we obtain

Lemma 14. $G$ is Mackey k-continuous for $k \in \mathbb{N} \sqcup\{$ lip, $\infty\}$ if and only if

$$
\mathrm{D}_{\mathrm{k}} \supseteq\left\{\phi_{n}\right\}_{n \in \mathbb{N}} \rightarrow_{\mathfrak{m} . \mathrm{k}} 0 \quad \Longrightarrow \quad \lim _{n}^{\infty} \int_{0}^{\bullet} \phi_{n}=e .
$$

The statement also holds for $k=\mathrm{c}$ if $G$ is abelian.

Proof. The one implication is evident. For the other implication, we suppose that $D_{\mathrm{k}} \supseteq\left\{\phi_{n}\right\}_{n \in \mathbb{N}} \rightarrow_{\mathfrak{m} . \mathrm{k}}$ $\phi \in \mathrm{D}_{\mathrm{k}}$ holds; and observe that

$$
\left[\int_{0}^{t} \phi\right]^{-1}\left[\int_{0}^{t} \phi_{n}\right] \stackrel{b]}{=} \int_{0}^{t} \underbrace{\operatorname{Ad}_{\left[\int_{0}^{\bullet} \phi\right]^{-1}}\left(\phi_{n}-\phi\right)}_{\psi_{n} \in \mathrm{D}_{\mathrm{k}}} \quad \forall n \in \mathbb{N}, t \in[0,1]
$$

holds by Point A), Then, by Lemma 6 and Lemma 7, we have $D_{\mathrm{k}} \supseteq\left\{\psi_{n}\right\}_{n \in \mathbb{N}} \rightarrow_{\mathfrak{m} . \mathrm{k}} 0$; from which the claim is clear.

We furthermore observe that

Lemma 15. If $G$ is Mackey k-continuous for $k \in \mathbb{N} \sqcup\{\mathrm{lip}, \infty, \mathrm{c}\}$, then $G$ is weakly k-continuous. 
Proof. If $G$ is not weakly k-continuous, then there exists an admissible $(\phi, \psi) \in C^{k}\left(\left[r, r^{\prime}\right], \mathfrak{g}\right) \times$ $C^{k}\left(\left[r, r^{\prime}\right], \mathfrak{g}\right)$, an open neighbourhood $U \subseteq G$ of $e$, as well as sequences $\left\{\tau_{n}\right\}_{n \in \mathbb{N}} \subseteq\left[r, r^{\prime}\right]$ and $\mathbb{R}_{\neq 0} \supseteq\left\{h_{n}\right\} \rightarrow 0$, such that

$$
\left[\int_{r}^{\tau_{n}} \phi\right]^{-1}\left[\int_{r}^{\tau_{n}} \phi+h_{n} \cdot \psi\right] \notin U \quad \forall n \in \mathbb{N}
$$

holds. Then, $G$ cannot be Mackey k-continuous, because we have $\left\{\phi+h_{n} \cdot \psi\right\}_{n \in \mathbb{N}} \rightarrow_{\mathfrak{m} . \mathrm{k}} \phi$.

Remarkably, the uniform convergence on the right side of (23) in Lemma 14 can be replaced by a weaker convergence property; namely,

Lemma 16. $G$ is Mackey k-continuous for $k \in \mathbb{N} \sqcup\{$ lip, $\infty\}$ if and only if

$$
\mathrm{D}_{\mathrm{k}} \supseteq\left\{\phi_{n}\right\}_{n \in \mathbb{N}} \rightarrow_{\mathfrak{m} . \mathrm{k}} 0 \quad \Longrightarrow \quad \lim _{n} \int \phi_{n}=e .
$$

The statement also holds for $k=\mathrm{c}$ if $G$ is abelian.

Proof. The one implication is evident. For the other implication, we suppose that (24) holds; and that $G$ is not Mackey k-continuous. By Lemma 14, there exist $D_{\mathrm{k}} \supseteq\left\{\phi_{n}\right\}_{n \in \mathbb{N}} \rightarrow_{\mathfrak{m} . \mathrm{k}} 0, U \subseteq G$ open with $e \in G$, a sequence $\left\{\tau_{n}\right\}_{n \in \mathbb{N}} \subseteq[0,1]$, and $\iota: \mathbb{N} \rightarrow \mathbb{N}$ strictly increasing, such that

$$
\int_{0}^{\tau_{n}} \phi_{\iota_{n}} \notin U \quad \forall n \in \mathbb{N}
$$

holds. For each $n \in \mathbb{N}$, we define

$$
\mathrm{D}_{\mathrm{k}} \ni \chi_{n}:=\dot{\varrho}_{n} \cdot\left(\phi_{\iota_{n}} \circ \varrho_{n}\right) \quad \text { with } \quad \varrho_{n}:[0,1] \ni t \mapsto t \cdot \tau_{n} \in\left[0, \tau_{n}\right] \text {; }
$$

and conclude from Remark 6 and Point B) that $\left\{\chi_{n}\right\}_{n \in \mathbb{N}} \rightarrow_{\mathfrak{m} . \mathrm{k}} 0$ holds. Then, (24) implies

$$
\lim _{n} \int_{0}^{\tau_{n}} \phi_{\iota_{n}} \stackrel{d]}{=} \lim _{n} \int \chi_{n}=e,
$$

which contradicts (25).

\subsection{Sequentially Continuity}

We write $\left\{\phi_{n}\right\}_{n \in \mathbb{N}} \rightarrow_{\mathfrak{s} . \mathrm{k}} \phi$ for $k \in \mathbb{N} \sqcup\{\operatorname{lip}, \infty, \mathrm{c}\},\left\{\phi_{n}\right\}_{n \in \mathbb{N}} \subseteq C^{k}\left(\left[r, r^{\prime}\right], \mathfrak{g}\right)$, and $\phi \in C^{k}\left(\left[r, r^{\prime}\right], \mathfrak{g}\right)$ if

$$
\lim _{n} \cdot \mathfrak{p}_{\infty}^{\mathrm{s}}\left(\phi-\phi_{n}\right)=0 \quad \forall \mathfrak{p} \in \mathfrak{P}, \mathrm{s} \preceq k
$$

holds. We say that $G$ is sequentially k-continuous if

$$
\mathrm{D}_{\mathrm{k}} \supseteq\left\{\phi_{n}\right\}_{n \in \mathbb{N}} \rightarrow_{\mathfrak{s . k}} \phi \in \mathrm{D}_{\mathrm{k}} \quad \Longrightarrow \quad \lim _{n}^{\infty} \int_{r}^{\bullet} \phi_{n}=\int_{r}^{\bullet} \phi .
$$

\section{Remark 7.}

1) Suppose that $G$ is sequentially k-continuous for $k \in \mathbb{N} \sqcup\{$ lip, $\infty, \mathrm{c}\}$. Evidently, then $G$ is Mackey $\mathrm{k}$-continuous; thus, weakly k-continuous by Lemma 15 .

2) $G$ is sequentially k-continuous for $k \in \mathbb{N} \sqcup\{$ lip, $\infty, \mathrm{c}\}$ if and only if

$$
\mathfrak{D}_{\left[r, r^{\prime}\right]}^{k} \supseteq\left\{\phi_{n}\right\}_{n \in \mathbb{N}} \rightarrow_{\mathfrak{s} . \mathrm{k}} \phi \in \mathfrak{D}_{\left[r, r^{\prime}\right]}^{k} \quad \Longrightarrow \quad \lim _{n}^{\infty} \int_{r}^{\bullet} \phi_{n}=\int_{r}^{\bullet} \phi
$$

holds for each $\left[r, r^{\prime}\right] \in \mathfrak{K}$. This just follows as in Lemma [13.

3) Let $k \in \mathbb{N} \sqcup\{$ lip, $\infty, \mathrm{c}\}$, with $G$ abelian for $k=\mathrm{c}$. Then, the same arguments as in Lemma 14 show that $G$ is sequentially k-continuous if and only if

$$
\mathrm{D}_{\mathrm{k}} \supseteq\left\{\phi_{n}\right\}_{n \in \mathbb{N}} \rightarrow_{\text {s.k }} 0 \quad \Longrightarrow \quad \lim _{n} \int_{0}^{\bullet} \phi_{n}=e .
$$


4) Let $k \in \mathbb{N} \sqcup\{$ lip, $\infty, \mathrm{c}\}$, with $G$ abelian for $k=\mathrm{c}$. Then, the same arguments as in Lemma 16 show that $G$ is sequentially k-continuous if and only if

$$
\mathrm{D}_{\mathrm{k}} \supseteq\left\{\phi_{n}\right\}_{n \in \mathbb{N}} \rightarrow_{\mathfrak{s . k}} 0 \quad \Longrightarrow \quad \lim _{n} \int \phi_{n}=e .
$$

5) If $G$ is $C^{k}$-continuous for $k \in \mathbb{N} \sqcup\{\operatorname{lip}, \infty, \mathrm{c}\}$, then $G$ is sequentially $\mathrm{k}$-continuous - This is clear

- for $k \in \mathbb{N} \sqcup\{$ lip, $\infty\} \quad$ from Point 4 ),

- for $k=\mathrm{c} \quad$ from Lemma 10 .

6) Let $k \in \mathbb{N} \sqcup\{$ lip, $\infty, \mathrm{c}\}$ be given; and suppose that the $C^{k}$-topology on $C^{k}([0,1], \mathfrak{g})$ is first countable, and that $G$ is sequentially k-continuous. Then, $G$ is $C^{k}$-continuous.

In fact, if $\mathrm{evol}_{\mathrm{k}}$ is not $C^{k}$-continuous, then there exists $U \subseteq G$ open, such that $W:=\operatorname{evol}_{\mathrm{k}}^{-1}(U) \subseteq$ $\mathrm{D}_{\mathrm{k}}$ is not open, i.e., not a neighbourhood of some $\phi \in W$. Since $C^{k}([0,1], \mathfrak{g})$ (thus, $\left.\mathrm{D}_{\mathrm{k}}\right)$ is first countable, there exists a sequence $\left\{\phi_{n}\right\}_{n \in \mathbb{N}} \subseteq \mathrm{D}_{\mathrm{k}} \backslash W$ with $\phi_{n} \rightarrow_{\text {s.k }} \phi$. We obtain $\operatorname{evol}_{\mathrm{k}}\left(\phi_{n}\right) \in$ $A:=G \backslash U$ for each $n \in \mathbb{N}$; thus, $\operatorname{evol}_{\mathrm{k}}(\phi)=\lim _{n} \operatorname{evol}_{\mathrm{k}}\left(\phi_{n}\right) \in A$, as $\mathrm{evol}_{\mathrm{k}}$ is sequentially continuous, and since $A$ is closed. This contradicts that $\operatorname{evol}_{\mathrm{k}}(\phi) \in U=G \backslash A$ holds.

\subsection{Piecewise Integrable Curves}

We now finally discuss piecewise integrable curves. Specifically, we provide the basic facts and definition:9; and furthermore show that sequentially 0-continuity and Mackey 0-continuity carry over to the piecewise integrable category. This will be used in Sect. 5 to generalize Theorem 1 in 8 .

\subsubsection{Basic Facts and Definitions}

For $k \in \mathbb{N} \sqcup\{$ lip, $\infty, \mathrm{c}\}$ and $\left[r, r^{\prime}\right] \in \mathfrak{K}$, we let $\mathfrak{D P}^{k}\left(\left[r, r^{\prime}\right], \mathfrak{g}\right)$ denote the set of all $\psi:\left[r, r^{\prime}\right] \rightarrow \mathfrak{g}$, such that there exist $r=t_{0}<\ldots<t_{n}=r^{\prime}$ and

$$
\psi[p] \in \mathfrak{D}_{\left[t_{p}, t_{p+1}\right]}^{k} \quad \text { with }\left.\quad \psi\right|_{\left(t_{p}, t_{p+1}\right)}=\left.\psi[p]\right|_{\left(t_{p}, t_{p+1}\right)} \quad \text { for } \quad p=0, \ldots, n-1 .
$$

In this situation, we define $\int_{r}^{r} \psi:=e$, as well as

$$
\int_{r}^{t} \psi:=\int_{t_{p}}^{t} \psi[p] \cdot \int_{t_{p-1}}^{t_{p}} \psi[p-1] \cdot \ldots \cdot \int_{t_{0}}^{t_{1}} \psi[0] \quad \forall t \in\left(t_{p}, t_{p+1}\right] .
$$

A standard refinement argument in combination with $c$ ) then shows that this is well defined; i.e., independent of any choices we have made. It is furthermore not hard to see that for $\phi, \psi \in$ $\mathfrak{D P}^{k}\left(\left[r, r^{\prime}\right], \mathfrak{g}\right)$, we have $\operatorname{Ad}_{\left[\int_{r}^{\bullet} \phi\right]^{-1}}(\psi-\phi) \in \mathfrak{D P}^{k}\left(\left[r, r^{\prime}\right], \mathfrak{g}\right)$ with

$$
\left[\int_{r}^{t} \phi\right]^{-1}\left[\int_{r}^{t} \psi\right]=\int_{r}^{t} \operatorname{Ad}_{\left[\int_{r}^{\bullet} \phi\right]^{-1}}(\psi-\phi) \quad \forall t \in\left[r, r^{\prime}\right] .
$$

We write

- $\left\{\phi_{n}\right\}_{n \in \mathbb{N}} \rightarrow_{\mathfrak{s}} \phi$ for $\left\{\phi_{n}\right\}_{n \in \mathbb{N}} \subseteq \mathfrak{D P}^{0}\left(\left[r, r^{\prime}\right], \mathfrak{g}\right)$ and $\phi \in \mathfrak{D P}^{0}\left(\left[r, r^{\prime}\right], \mathfrak{g}\right)$ if

$$
\lim _{n} \cdot \mathfrak{p}_{\infty}\left(\phi-\phi_{n}\right)=0 \quad \forall \mathfrak{p} \in \mathfrak{P}
$$

holds.

- $\left\{\phi_{n}\right\}_{n \in \mathbb{N}} \rightarrow_{\mathfrak{m}} \phi$ for $\left\{\phi_{n}\right\}_{n \in \mathbb{N}} \subseteq \mathfrak{D P}^{0}\left(\left[r, r^{\prime}\right], \mathfrak{g}\right)$ and $\phi \in \mathfrak{D P}^{0}\left(\left[r, r^{\prime}\right], \mathfrak{g}\right)$ if

$$
\mathfrak{p}_{\infty}\left(\phi-\phi_{n}\right) \leq \mathfrak{c}_{\mathfrak{p}} \cdot \lambda_{n} \quad \forall n \geq \mathfrak{l}_{\mathfrak{p}}, \mathfrak{p} \in \mathfrak{P}
$$

holds for certain $\left\{\mathfrak{c}_{\mathfrak{p}}\right\}_{\mathfrak{p} \in \mathfrak{P}} \subseteq \mathbb{R}_{\geq 0},\left\{\mathfrak{l}_{\mathfrak{p}}\right\}_{\mathfrak{p} \in \mathfrak{P}} \subseteq \mathbb{N}$, and $\mathbb{R}_{\geq 0} \supseteq\left\{\lambda_{n}\right\}_{n \in \mathbb{N}} \rightarrow 0$.

${ }^{9}$ Confer Sect. 4.3 in [7] for the statements mentioned but not proven here. 


\subsubsection{A Continuity Statement}

We recall the construction made in Sect. 4.3 in [7].

i) We fix (a bump function) $\boldsymbol{\rho}:[0,1] \rightarrow[0,2]$ smooth with

$$
\left.\boldsymbol{\rho}\right|_{(0,1)}>0, \int_{0}^{1} \boldsymbol{\rho}(s) \mathrm{d} s=1 \quad \text { as well as } \quad \boldsymbol{\rho}^{(k)}(0)=0=\boldsymbol{\rho}^{(k)}(1) \quad \forall k \in \mathbb{N} .
$$

Then, given $\left[r, r^{\prime}\right] \in \mathfrak{K}$ and $r=t_{0}<\ldots<t_{n}=r^{\prime}$, we let

$$
\rho_{p}:\left[t_{p}, t_{p+1}\right] \rightarrow[0,2], \quad t \mapsto \boldsymbol{\rho}\left(\left(t-t_{p}\right) /\left(t_{p+1}-t_{p}\right)\right) \quad \forall p=0, \ldots, n-1 ;
$$

and define $\rho:\left[r, r^{\prime}\right] \rightarrow[0,2]$ by

$$
\left.\rho\right|_{\left[t_{p}, t_{p+1}\right]}:=\rho_{p} \quad \forall p=0, \ldots, n-1 .
$$

Then, $\rho$ is smooth, with $\rho^{(k)}\left(t_{p}\right)=0$ for each $k \in \mathbb{N}, p=0, \ldots, n$; and (10) shows that

$$
\varrho:\left[r, r^{\prime}\right] \rightarrow\left[r, r^{\prime}\right], \quad t \mapsto r+\int_{r}^{t} \rho(s) \mathrm{d} s
$$

holds, with $\varrho\left(t_{p}\right)=t_{p}$ for $p=0, \ldots, n-1$.

ii) For $\psi \in \mathfrak{D P}^{0}\left(\left[r, r^{\prime}\right], \mathfrak{g}\right)$ with $r=t_{0}<\ldots<t_{n}=r^{\prime}$ as well as $\psi[0], \ldots, \psi[n-1]$ as in (26), we let $\varrho:\left[r, r^{\prime}\right] \rightarrow\left[r, r^{\prime}\right]$ and $\rho \equiv \dot{\varrho}:\left[r, r^{\prime}\right] \rightarrow[0,2]$ be as in i). Then, it is straightforward from the definitions that

$$
\chi:=\rho \cdot(\psi \circ \varrho) \in \mathfrak{D}_{\left[r, r^{\prime}\right]}^{0} \quad \text { holds, with } \quad \int_{r}^{\varrho} \psi=\int_{r}^{\bullet} \chi \quad \text { and } \quad \mathfrak{p}_{\infty}(\chi) \leq 2 \cdot \mathfrak{p}_{\infty}(\psi)
$$

for each $\mathfrak{p} \in \mathfrak{P} 10$

We obtain

\section{Lemma 17.}

1) If $G$ is sequentially 0 -continuous, then

$$
\mathfrak{D P}^{0}\left(\left[r, r^{\prime}\right], \mathfrak{g}\right) \supseteq\left\{\phi_{n}\right\}_{n \in \mathbb{N}} \rightarrow_{\mathfrak{s}} \phi \in \mathfrak{D P}^{0}\left(\left[r, r^{\prime}\right], \mathfrak{g}\right) \quad \Longrightarrow \quad \lim _{n}^{\infty} \int_{r}^{\bullet} \phi_{n}=\int_{r}^{\bullet} \phi .
$$

2) If $G$ is Mackey 0-continuous, then

$$
\mathfrak{D P} P^{0}\left(\left[r, r^{\prime}\right], \mathfrak{g}\right) \supseteq\left\{\phi_{n}\right\}_{n \in \mathbb{N}} \rightarrow_{\mathfrak{m}} \phi \in \mathfrak{D P}^{0}\left(\left[r, r^{\prime}\right], \mathfrak{g}\right) \quad \Longrightarrow \quad \lim _{n}^{\infty} \int_{r}^{\bullet} \phi_{n}=\int_{r}^{\bullet} \phi .
$$

Specifically, in both situations, for each $\mathfrak{p} \in \mathfrak{P}$, there exist some $\mathfrak{p} \leq \mathfrak{q} \in \mathfrak{P}$ and $n_{\mathfrak{p}} \in \mathbb{N}$ with

$$
(\mathfrak{p} \circ \Xi)\left(\left[\int_{r}^{\bullet} \phi\right]^{-1}\left[\int_{r}^{\bullet} \phi_{n}\right]\right) \leq \int \mathfrak{q}\left(\phi_{n}(s)-\phi(s)\right) \mathrm{d} s \quad \forall n \geq n_{\mathfrak{p}} .
$$

Proof. Let $\phi \in \mathfrak{D P}^{0}\left(\left[r, r^{\prime}\right], \mathfrak{g}\right)$ and $\left\{\phi_{n}\right\}_{n \in \mathbb{N}} \subseteq \mathfrak{D P}^{0}\left(\left[r, r^{\prime}\right], \mathfrak{g}\right)$ be given. For $\mathfrak{p} \equiv \mathfrak{u} \in \mathfrak{P}$ fixed, we choose $U \subseteq G$ and $\mathfrak{u} \leq \mathfrak{m} \in \mathfrak{P}$ as in Lemma 4. We furthermore let $\mathfrak{m} \leq \mathfrak{q} \equiv \mathfrak{w} \in \mathfrak{P}$ be as in I), for $\mathrm{C} \equiv \operatorname{im}\left[\left[\int_{r}^{\bullet} \phi\right]^{-1}\right]$ and $\mathfrak{v} \equiv \mathfrak{m}$ there. Then, for each $n \in \mathbb{N}$, we let $\varrho_{n} \equiv \varrho, \rho_{n} \equiv \rho$, and $\chi_{n} \equiv \chi$ be as in ii), for

$$
\psi \equiv \psi_{n}:=\operatorname{Ad}_{\left[\int_{r}^{\bullet} \phi\right]^{-1}}\left(\phi_{n}-\phi\right) \in \mathfrak{D P}^{0}\left(\left[r, r^{\prime}\right], \mathfrak{g}\right)
$$

there. Then,

- we have

$$
\left[\int_{r}^{\varrho_{n}(t)} \phi\right]^{-1}\left[\int_{r}^{\varrho_{n}(t)} \phi_{n}\right] \stackrel{[28)}{=} \int_{r}^{\varrho_{n}(t)} \operatorname{Ad}_{\left[\int_{r}^{\bullet} \phi\right]^{-1}}\left(\phi_{n}-\phi\right) \stackrel{\text { ii) }}{=} \int_{r}^{t} \chi_{n} \quad \forall n \in \mathbb{N}, t \in\left[r, r^{\prime}\right] .
$$

\footnotetext{
${ }^{10}$ In the proof of Lemma 24 in [7, this statement was more generally verified for the case that $k \in \mathbb{N} \sqcup\{$ lip, $\infty\}$ holds.
} 
- we have $\mathfrak{m}_{\infty}\left(\chi_{n}\right) \leq 2 \cdot \mathfrak{m}_{\infty}\left(\psi_{n}\right) \leq 2 \cdot \mathfrak{q}_{\infty}\left(\phi_{n}-\phi\right)$ for each $n \in \mathbb{N}$ by Lemma 6 , which shows that - $\mathfrak{D}_{\left[r, r^{\prime}\right]}^{0} \supseteq\left\{\chi_{n}\right\}_{n \in \mathbb{N}} \rightarrow_{\mathfrak{s} .0} 0$ holds if we are in the situation of 1 ),

- $\mathfrak{D}_{\left[r, r^{\prime}\right]}^{0} \supseteq\left\{\chi_{n}\right\}_{n \in \mathbb{N}} \rightarrow_{\mathfrak{m} .0} 0$ holds if we are in the situation of 2 ),

In both situations, there thus exists some $n_{\mathfrak{p}} \in \mathbb{N}$ with $\int_{r}^{\bullet} \chi_{n} \in U$ for each $n \geq n_{\mathfrak{p}}$.

We obtain from Lemma 4 (second step), and I) (last step) that 11

$$
\begin{aligned}
(\mathfrak{p} \circ \Xi)\left(\left[\int_{r}^{\varrho_{n}(t)} \phi\right]^{-1}\left[\int_{r}^{\varrho_{n}(t)} \phi_{n}\right]\right) & \stackrel{(30)}{=}(\mathfrak{p} \circ \Xi)\left(\int_{r}^{t} \chi_{n}\right) \\
& \leq \int_{r}^{t}\left(\mathfrak{m} \circ \chi_{n}\right)(s) \mathrm{d} s \\
& \stackrel{(10)}{=} \int_{r}^{\varrho_{n}(t)}\left(\cdot \mathfrak{m} \circ \psi_{n}\right)(s) \mathrm{d} s \\
& \leq \int_{r}^{\varrho_{n}(t)} \cdot \mathfrak{q}\left(\phi_{n}(s)-\phi(s)\right) \mathrm{d} s
\end{aligned}
$$

holds for all $n \geq n_{\mathfrak{p}}$ and $t \in\left[r, r^{\prime}\right]$; which proves the claim.

\section{Mackey Continuity}

In this section, we show that

Theorem 1. If $G$ is $C^{k}$-semiregular for $k \in \mathbb{N} \sqcup\{$ lip, $\infty\}$, then $G$ is Mackey k-continuous.

The proof of Theorem 1 is based on a bump function argument similar to that one used in the proof of Theorem 2 in [7]. It furthermore makes use of the fact that $[0,1] \ni t \mapsto \int_{0}^{t} \phi \in G$ is continuous for each $\phi \in \mathrm{D}_{\mathrm{k}}$. However, before we can provide the proof, we need some technical preparation first.

\subsection{Some Estimates}

Suppose we are given $\varrho:\left[r, r^{\prime}\right] \rightarrow\left[r, r^{\prime}\right]$; and let $\rho \equiv \dot{\varrho}$ as well as

$$
C[\rho, \mathrm{s}]:=\max \left(1, \max _{0 \leq m, n \leq \mathrm{s}}\left(\sup \left\{\left|\rho^{(m)}(t)\right|^{n+1} \mid t \in\left[r, r^{\prime}\right]\right\}\right)\right) \quad \forall \mathrm{s} \in \mathbb{N} .
$$

We observe the following:

- Let $\psi \in C^{k}\left(\left[r, r^{\prime}\right], \mathfrak{g}\right)$ for $k \in \mathbb{N} \sqcup\{\infty\}$ and $\mathrm{s} \preceq k$ be given. By c), d) in Appendix A.1, we have

$$
(\rho \cdot(\psi \circ \varrho))^{(\mathrm{s})}=\sum_{q, m, n=0}^{\mathrm{s}} h_{\mathrm{s}}(q, m, n) \cdot\left(\rho^{(m)}\right)^{n+1} \cdot\left(\psi^{(q)} \circ \varrho\right),
$$

for a map $h_{\mathrm{s}}:(0, \ldots, \mathrm{s})^{3} \rightarrow\{0,1\}$ that is independent of $\varrho, \rho, \phi 12$ We obtain

$$
\mathfrak{p}\left((\rho \cdot(\psi \circ \varrho))^{(\mathrm{s})}\right) \leq(\mathrm{s}+1)^{3} \cdot C[\rho, \mathrm{s}] \cdot \mathfrak{p}_{\infty}^{\mathrm{s}}(\psi)
$$

for each $\mathfrak{p} \in \mathfrak{P}, 0 \leq \mathrm{s} \preceq k$, and $\psi \in C^{k}\left(\left[r, r^{\prime}\right], \mathfrak{g}\right)$.

- Let $\psi \in C^{\operatorname{lip}}\left(\left[r, r^{\prime}\right], \mathfrak{g}\right)$ be given. Then we have

$$
\begin{aligned}
\mathfrak{p}((\rho \cdot(\psi \circ \varrho))(t) & \left.-(\rho \cdot(\psi \circ \varrho))\left(t^{\prime}\right)\right) \\
& \leq\left|\rho(t)-\rho\left(t^{\prime}\right)\right| \cdot \mathfrak{p}(\psi(\varrho(t)))+\left|\rho\left(t^{\prime}\right)\right| \cdot \mathfrak{p}\left(\psi(\varrho(t))-\psi\left(\varrho\left(t^{\prime}\right)\right)\right) \\
& \leq\left|t-t^{\prime}\right| \cdot C[\rho, 1] \cdot \mathfrak{p}_{\infty}(\psi)+C[\rho, 0] \cdot \operatorname{Lip}(\mathfrak{p}, \psi) \cdot \underbrace{\left|\varrho(t)-\varrho\left(t^{\prime}\right)\right|}_{\leq C[\rho, 0] \cdot\left|t-t^{\prime}\right|} \\
& \leq 2 \cdot C[\rho, 1]^{2} \cdot \mathfrak{p}_{\infty}^{\operatorname{lip}}(\psi) \cdot\left|t-t^{\prime}\right|
\end{aligned}
$$

\footnotetext{
${ }^{11}$ For the third step observe that $\rho_{n} \geq 0$ holds for each $n \in \mathbb{N}$.

${ }^{12}$ More concretely, $h_{\mathrm{s}}(q, m, n)$ are the coefficients appearing in the Leibniz rule for iterated derivatives of compositions.
} 
for each $t, t^{\prime} \in\left[r, r^{\prime}\right]$; thus,

$$
\operatorname{Lip}(\cdot \mathfrak{p}, \rho \cdot(\psi \circ \varrho)) \leq 2 \cdot C[\rho, 1]^{2} \cdot \mathfrak{p}_{\infty}^{\operatorname{lip}}(\psi) .
$$

Let now $\boldsymbol{\rho}:[0,1] \rightarrow[0,2]$ be a fixed bump function as in (29); as well as $\left\{t_{n}\right\}_{n \in \mathbb{N}} \subseteq[0,1]$ strictly decreasing with $t_{0}=1$. For each $n \in \mathbb{N}$, we define $0<\delta_{n}:=t_{n}-t_{n+1}<1$, as well as

$$
\rho_{n}:=\delta_{n}^{-1} \cdot\left(\rho \circ \kappa_{n}\right) \quad \text { for } \quad \kappa_{n}:\left[t_{n+1}, t_{n}\right] \ni t \mapsto \delta_{n}^{-1} \cdot\left(t-t_{n+1}\right) \in[0,1] .
$$

We obtain from (10) that

$$
\varrho_{n}:\left[t_{n+1}, t_{n}\right] \ni t \mapsto \int_{t_{n+1}}^{t} \rho_{n}(s) \mathrm{d} s \in[0,1] \quad \forall n \in \mathbb{N}
$$

holds; and furthermore observe that

$$
C\left[\rho_{n}, \mathrm{~s}\right] \leq \delta_{n}^{-(\mathrm{s}+1)^{2}} \cdot C[\boldsymbol{\rho}, \mathrm{s}] \quad \forall n \in \mathbb{N} .
$$

\subsection{A Construction}

Suppose we are given $\left\{\phi_{n}\right\}_{n \in \mathbb{N}} \subseteq \mathfrak{D}_{[0,1]}^{k}$ with $k \in \mathbb{N} \sqcup\{$ lip, $\infty\}$; and let $\boldsymbol{\rho}, \rho_{n}, \varrho_{n},\left\{\tau_{n}\right\}_{n \in \mathbb{N}},\left\{\delta_{n}\right\}_{n \in \mathbb{N}}$ be as in Sect. 4.1, Then,

- We obtain from (31) and (33) that

$$
\begin{aligned}
\cdot \mathfrak{p}\left(\left(\rho_{n} \cdot\left(\phi_{n} \circ \varrho_{n}\right)\right)^{(\mathrm{s})}\right) & \stackrel{\sqrt{311}}{\leq}(\mathrm{s}+1)^{3} \cdot C\left[\rho_{n}, \mathrm{~s}\right] \cdot \mathfrak{p}_{\infty}^{\mathrm{s}}\left(\phi_{n}\right) \\
& \stackrel{\sqrt{33})}{\leq}(\mathrm{s}+1)^{3} \cdot \delta_{n}^{-(\mathrm{s}+1)^{2}} \cdot C[\boldsymbol{\rho}, \mathrm{s}] \cdot \mathfrak{p}_{\infty}^{\mathrm{s}}\left(\phi_{n}\right)
\end{aligned}
$$

holds, for each $\mathfrak{p} \in \mathfrak{P}, \mathrm{s} \preceq k$, and $n \in \mathbb{N}$.

- We obtain from (32) and (33) that

$$
\operatorname{Lip}\left(\cdot \mathfrak{p}, \rho_{n} \cdot\left(\phi_{n} \circ \varrho_{n}\right)\right) \stackrel{(32)}{\leq} 2 \cdot C\left[\rho_{n}, 1\right]^{2} \cdot \mathfrak{p}_{\infty}^{\mathrm{lip}}\left(\phi_{n}\right) \stackrel{\sqrt{33})}{\leq} 2 \cdot \delta_{n}^{-8} \cdot C[\boldsymbol{\rho}, 1]^{2} \cdot \mathfrak{p}_{\infty}^{\mathrm{lip}}\left(\phi_{n}\right)
$$

holds, for each $\mathfrak{p} \in \mathfrak{P}$ and $n \in \mathbb{N}$.

We define $\phi:[0,1] \rightarrow \mathfrak{g}$, by

$$
\phi(0):=0 \quad \text { and }\left.\quad \phi\right|_{\left[t_{n+1}, t_{n}\right]}:=\rho_{n} \cdot\left(\phi_{n} \circ \varrho_{n}\right) \quad \forall n \in \mathbb{N} .
$$

Then, it is straightforward to see that 13||$_{\left[t_{n+1}, 1\right]} \in \mathfrak{D}_{\left[t_{n+1}, 1\right]}^{k}$ holds for each $n \in \mathbb{N}$, with

$$
\left.\int_{0}^{\varrho_{n}} \phi_{n} \stackrel{d]}{=} \int_{t_{n+1}}^{\bullet} \phi\right|_{\left[t_{n+1}, t_{n}\right]} \quad \forall n \in \mathbb{N} .
$$

Moreover, for $k=$ lip, we obtain from (35) that

$$
\operatorname{Lip}\left(\mathfrak{p},\left.\phi\right|_{\left[t_{n+1}, 1\right]}\right) \leq 2 \cdot C[\boldsymbol{\rho}, 1]^{2} \cdot \max \left(\delta_{0}^{-8} \cdot \mathfrak{p}_{\infty}^{\operatorname{lip}}\left(\phi_{0}\right), \ldots, \delta_{n}^{-8} \cdot \mathfrak{\cdot} \mathfrak{p}_{\infty}^{\operatorname{lip}}\left(\phi_{n}\right)\right)
$$

holds, cf. Appendix A.3.

\footnotetext{
${ }^{13}$ The technical details can be found, e.g., in the proof of Lemma 24 in 7 .
} 


\subsection{Proof of Theorem 1}

We are ready for the

Proof of Theorem 1. Suppose that the claim is wrong, i.e., that $G$ is $C^{k}$-semiregular for $k \in$ $\mathbb{N} \sqcup\{$ lip, $\infty\}$ but not Mackey k-continuous. Then, by Lemma 14, there exists a sequence $D_{k} \supseteq$ $\left\{\phi_{n}\right\}_{n \in \mathbb{N}} \rightarrow_{\mathfrak{m} . \mathrm{k}} 0$ (with $\left\{\mathfrak{c}_{\mathfrak{p}}^{\mathrm{s}}\right\}_{\mathrm{s} \prec k, \mathfrak{p} \in \mathfrak{P}} \subseteq \mathbb{R}_{\geq 0},\left\{\mathfrak{l}_{\mathfrak{p}}^{\mathrm{s}}\right\}_{\mathbf{s} \preceq k, \mathfrak{p} \in \mathfrak{P}} \subseteq \mathbb{N}$, and $\mathbb{R}_{\geq 0} \supseteq\left\{\lambda_{n}\right\}_{n \in \mathbb{N}} \rightarrow 0$ as in (21) for $\phi \equiv 0$ there), as well as $U \subseteq G$ open with $e \in U$, such that $\operatorname{im}\left[\int_{0}^{\bullet} \phi_{n}\right] \nsubseteq \subseteq U$ holds for infinitely many $n \in \mathbb{N}$. Passing to a subsequence if necessary, we thus can assume that

$$
\operatorname{im}\left[\int_{0}^{\bullet} \phi_{n}\right] \not \subseteq U \quad \text { and } \quad \lambda_{n} \leq 2^{-(n+1)^{2}} \quad \forall n \in \mathbb{N}
$$

holds. We let $t_{0}:=1$, and $t_{n}:=1-\sum_{k=1}^{n} 2^{-k}$ for each $n \in \mathbb{N}_{\geq 1}$; so that $\delta_{n}=t_{n}-t_{n+1}=2^{-(n+1)}$ holds for each $n \in \mathbb{N}$. We construct $\phi:[0,1] \rightarrow \mathfrak{g}$ as in (36) in Sect. 4.2, and fix $V \subseteq U$ open with $e \in V$ and $V \cdot V^{-1} \subseteq U$.

Suppose now that we have shown that $\phi$ is of class $C^{k}$; i.e., that $\phi \in \mathrm{D}_{\mathrm{k}}$ holds as $G$ is $C^{k}$-semiregular. Since $[0,1] \ni t \mapsto \int_{0}^{t} \phi \in G$ is continuous, there exists some $\ell \geq 1$ with $\int_{0}^{t} \phi \in V$ for each $t \in\left[0, t_{\ell}\right]$; thus,

$$
\left.\int_{0}^{\varrho_{n}(t)} \phi_{n} \stackrel{(37)}{=} \int_{t_{n+1}}^{t} \phi\right|_{\left[t_{n+1}, t_{n}\right]} \stackrel{[)]}{=}\left[\int_{0}^{t} \phi\right] \cdot\left[\int_{0}^{t_{n+1}} \phi\right]^{-1} \in V \cdot V^{-1} \subseteq U
$$

for each $t \in\left[t_{n+1}, t_{n}\right]$ with $n \geq \ell$, which contradicts (39).

To prove the claim, it thus suffices to show that $\phi$ is of class $C^{k}$ :

- Suppose first that $k \in \mathbb{N} \sqcup\{\infty\}$ holds. Then, it suffices to show that

$$
\lim _{(0,1] \ni h \rightarrow 0} 1 / h \cdot \phi^{(\mathrm{s})}(h)=0 \quad \forall \mathrm{s} \preceq k
$$

holds, because $\phi$ is of class $C^{k}$ on $(0,1]$.

For this, we let $h \in\left[t_{n+1}, t_{n}\right]$ for $n \in \mathbb{N}$ be given; and observe that

$$
h \geq t_{n}=t_{n}-t_{n+1}+t_{n+1}=2^{-(n+1)}+1-\sum_{k=1}^{n+1} 2^{-k} \geq 2^{-(n+1)}
$$

holds. Then, for $\mathfrak{p} \in \mathfrak{P}$ fixed and $n \geq \mathfrak{l}_{\mathfrak{p}}^{\mathrm{s}}$, we obtain from (34) $\left(\right.$ with $\left.\delta_{n}=2^{-(n+1)}\right)$ as well as (39) that

$$
\begin{aligned}
1 / h \cdot \mathfrak{p}\left(\phi^{(\mathrm{s})}(h)\right) & \leq 2^{n+1} \cdot \mathfrak{p}\left(\left(\rho_{n} \cdot\left(\phi_{n} \circ \varrho_{n}\right)\right)^{(\mathrm{s})}\right) \\
& \stackrel{(34)}{\leq}(\mathrm{s}+1)^{3} \cdot 2^{\left((\mathrm{s}+1)^{2}+1\right) \cdot(n+1)} \cdot C[\boldsymbol{\rho}, \mathrm{s}] \cdot \mathfrak{p}_{\infty}^{\mathrm{s}}\left(\phi_{n}\right) \\
& \stackrel{(39))}{\leq}(\mathrm{s}+1)^{3} \cdot C[\boldsymbol{\rho}, \mathrm{s}] \cdot \mathfrak{c}_{\mathfrak{p}}^{\mathrm{s}} \cdot 2^{\left((\mathrm{s}+1)^{2}+1\right) \cdot(n+1)-(n+1)^{2}} \\
& =(\mathrm{s}+1)^{3} \cdot C[\boldsymbol{\rho}, \mathrm{s}] \cdot \mathfrak{c}_{\mathfrak{p}}^{\mathrm{s}} \cdot 2^{\left(\left((\mathrm{s}+1)^{2}+1\right)-(n+1)\right) \cdot(n+1)}
\end{aligned}
$$

holds; which clearly tends to zero for $n \rightarrow \infty$.

- Suppose now that $k=$ lip holds. The previous point then shows $\phi \in C^{0}([0,1], \mathfrak{g})$. For $\mathfrak{p} \in \mathfrak{P}$ fixed, we thus have $\mathfrak{p}_{\infty}(\phi)<\infty$. We let $\mathfrak{l}_{\mathfrak{p}} \equiv \mathfrak{l}_{\mathfrak{p}}^{\text {lip }}$ for each $\mathfrak{p} \in \mathfrak{P}$, define

$$
D_{\mathfrak{p}}:=\max \left(2 \cdot \delta_{0}^{-8} \cdot \mathfrak{p}_{\infty}^{\operatorname{lip}}\left(\phi_{0}\right), \ldots, 2 \cdot \delta_{\mathfrak{l}_{\mathfrak{p}}}^{-8} \cdot \mathfrak{p}_{\infty}^{\operatorname{lip}}\left(\phi_{\mathfrak{l}_{\mathfrak{p}}}\right)\right)
$$


and obtain for $n \geq \mathfrak{l}_{\mathfrak{p}}$ that

$$
\begin{aligned}
\operatorname{Lip}\left(\cdot \mathfrak{p},\left.\phi\right|_{\left[t_{n+1}, 1\right]}\right) & \stackrel{(38)}{\leq} C[\boldsymbol{\rho}, 1]^{2} \cdot \max \left(2 \cdot \delta_{0}^{-8} \cdot \mathfrak{p}_{\infty}^{\operatorname{lip}}\left(\phi_{0}\right), \ldots, 2 \cdot \delta_{n}^{-8} \cdot \mathfrak{p}_{\infty}^{\operatorname{lip}}\left(\phi_{n}\right)\right) \\
& \stackrel{(21)}{\leq} C[\boldsymbol{\rho}, 1]^{2} \cdot \max \left(D_{\mathfrak{p}}, \mathfrak{c}_{\mathfrak{p}}^{\operatorname{lip}} \cdot \max \left(2^{1+8\left(\mathfrak{l}_{\mathfrak{p}}+2\right)} \cdot \lambda_{\mathfrak{l}_{\mathfrak{p}}+1}, \ldots, 2^{1+8(n+1)} \cdot \lambda_{n}\right)\right) \\
& \stackrel{(39)}{\leq} C[\boldsymbol{\rho}, 1]^{2} \cdot \max \left(D_{\mathfrak{p}}, \mathfrak{c}_{\mathfrak{p}}^{\operatorname{lip}} \cdot \max \left\{2^{1+8(\ell+1)} \cdot 2^{-(\ell+1)^{2}} \mid \mathfrak{l}_{\mathfrak{p}}+1 \leq \ell \leq n\right\}\right) \\
& =C[\boldsymbol{\rho}, 1]^{2} \cdot \max \left(D_{\mathfrak{p}}, \mathfrak{c}_{\mathfrak{p}}^{\operatorname{lip}} \cdot \max \left\{2^{-\ell^{2}+6 \ell+8} \mid \mathfrak{l}_{\mathfrak{p}}+1 \leq \ell \leq n\right\}\right) \\
& =C[\boldsymbol{\rho}, 1]^{2} \cdot \max \left(D_{\mathfrak{p}}, \mathfrak{c}_{\mathfrak{p}}^{\operatorname{lip}} \cdot \max \left\{2^{17-(\ell-3)^{2}} \mid \mathfrak{l}_{\mathfrak{p}}+1 \leq \ell \leq n\right\}\right) \\
& \leq C[\boldsymbol{\rho}, 1]^{2} \cdot \max \left(D_{\mathfrak{p}}, \mathfrak{c}_{\mathfrak{p}} \operatorname{lip}^{\text {lip }} \cdot 2^{17}\right)=: \mathfrak{L}
\end{aligned}
$$

holds; thus,

$$
\mathfrak{p}\left(\phi(t)-\phi\left(t^{\prime}\right)\right) \leq \mathfrak{L} \cdot\left|t-t^{\prime}\right| \quad \forall t, t^{\prime} \in(0,1] .
$$

Moreover, since $\phi$ is continuous with $\phi(0)=0$, for each $t \in[0,1]$ we have

$$
\begin{aligned}
\mathfrak{p}(\phi(0)-\phi(t)) & =\lim _{\ell \rightarrow \infty} \mathfrak{p}(\phi(0)-\phi(1 / \ell)+\phi(1 / \ell)-\phi(t)) \\
& \leq \lim _{\ell \rightarrow \infty} \cdot \mathfrak{p}(\phi(1 / \ell))+\lim _{\ell \rightarrow \infty} \cdot \mathfrak{p}(\phi(1 / \ell)-\phi(t)) \\
& =\lim _{\ell \rightarrow \infty} \mathfrak{p}(\phi(1 / \ell)-\phi(t)) \\
& \leq \lim _{\ell \rightarrow \infty} \mathfrak{L} \cdot|1 / \ell-t| \\
& =\mathfrak{L} \cdot|0-t| .
\end{aligned}
$$

This shows $\operatorname{Lip}(\mathfrak{p}, \phi) \leq \mathfrak{L}$, i.e., $\phi \in C^{\operatorname{lip}}([0,1], \mathfrak{g})$.

\section{The Strong Trotter Property}

In this Section, we want to give a brief application of the notions introduced so far. For this, we recall that a Lie group $G$ is said to have the strong Trotter property if (1) holds; and now will show

Proposition 1. 1) If $G$ is sequentially 0-continuous, then $G$ has the strong Trotter property.

2) If $G$ is Mackey 0-continuous, then (1) holds for each $\mu \in C_{*}^{1}([0,1], G)$ with $\dot{\mu}(0) \in \operatorname{dom}[\exp ]$ and $\delta^{r}(\mu) \in C^{\operatorname{lip}}([0,1], \mathfrak{g})$.

Here,

- By Remark 75), Proposition 14) generalizes Theorem 1 in [8], stating that $G$ admits the strong Trotter property if $G$ is locally $\mu$-convex (recall that, by Theorem 1 in [7], local $\mu$-convexity is equivalent to that $G$ is $C^{0}$-continuous).

- By Theorem 1, the presumptions made in Proposition 12) are always fulfilled, e.g., if $G$ is $C^{0}$-semiregular, and $\mu$ is of class $C^{2}$ with $\dot{\mu}(0) \in \operatorname{dom}[\exp ]$.

We will need the following observations: Let $\ell>0, \mu \in C_{*}^{1}([0,1], \mathcal{U})$ be given; and define $\phi:=\delta^{r}(\mu)$, $X:=\dot{\mu}(0)=\phi(0)$, as well as

$$
\mu_{\tau}:[0,1 / \ell] \ni t \mapsto \mu(\tau \cdot t) \in G \quad \forall \tau \in[0, \ell] .
$$

Then, for each $t \in[0,1 / \ell]$, we have

$$
\begin{aligned}
\delta^{r}\left(\mu_{\tau}\right)(t)-\tau \cdot X & \stackrel{(14)}{=} \omega\left(\left(\Xi \circ \mu_{\tau}\right)(t), \partial_{t}\left(\Xi \circ \mu_{\tau}\right)(t)\right)-\tau \cdot X \\
& =\tau \cdot \omega\left((\Xi \circ \mu)(\tau \cdot t), \partial_{t}(\Xi \circ \mu)(\tau \cdot t)\right)-\tau \cdot X \\
& =\tau \cdot \delta^{r}(\mu)(\tau \cdot t)-\tau \cdot X \\
& =\tau \cdot(\phi(\tau \cdot t)-X) .
\end{aligned}
$$


For each $\mathfrak{p} \in \mathfrak{P}, \tau \in[0, \ell]$, and $s \leq 1 / \ell$, we thus obtain

$$
\mathfrak{p}_{\infty}\left(\left.\delta^{r}\left(\mu_{\tau}\right)\right|_{[0, s]}-\tau \cdot X\right)=\ell \cdot \sup \{\mathfrak{p}(\phi(\tau \cdot t)-\phi(0)) \mid t \in[0, s]\} ;
$$

whereby, for the case that $\phi \in C^{\mathrm{lip}}([0,1], \mathfrak{g})$ holds, we additionally have

$$
\sup \{\mathfrak{p}(\phi(\tau \cdot t)-\phi(0)) \mid t \in[0, s]\} \leq s \cdot \ell \cdot \operatorname{Lip}(\cdot \mathfrak{p}, \phi)
$$

We are ready for the

Proof of Proposition 1, Let $\phi:=\delta^{r}(\mu)$ and $X:=\dot{\mu}(0)=\phi(0)$, for

1) $\mu \in C_{*}^{1}([0,1], G)$ with $\dot{\mu}(0) \in \operatorname{dom}[\exp ]$ if $G$ is sequentially 0-continuous.

2) $\mu \in C_{*}^{1}([0,1], G)$ with $\dot{\mu}(0) \in \operatorname{dom}[\exp ]$ and $\phi \in C^{\text {lip }}([0,1], \mathfrak{g})$ if $G$ is Mackey 0-continuous.

We suppose that (11) is wrong; i.e., that there exists some $\ell>0$, an open neighbourhood $U \subseteq G$ of $e$, a sequence $\left\{\tau_{n}\right\}_{n \in \mathbb{N}} \subseteq[0, \ell]$, and a strictly increasing sequence $\left\{\iota_{n}\right\}_{n \in \mathbb{N}} \subseteq \mathbb{N}_{\geq 1} \cap[\ell, \infty)$ with

$$
\exp \left(-\tau_{n} \cdot X\right) \cdot \mu\left(\tau_{n} / \iota_{n}\right)^{\iota_{n}} \notin U \quad \forall n \in \mathbb{N} .
$$

Passing to a subsequence if necessary, we can additionally assume that $\lim _{n} \tau_{n}=\tau \in[0, \ell]$ exists. We choose $V \subseteq G$ open with $e \in V$ and $V \cdot V \subseteq U$, and fix some $n_{V} \in \mathbb{N}$ with

$$
\exp \left(\left(\tau-\tau_{n}\right) \cdot X\right) \in V \quad \forall n \geq n_{V} .
$$

Moreover, for each $n \in \mathbb{N}$ :

- We define

$$
\chi_{n}:=\left.\delta^{r}\left(\mu_{\tau_{n}}\right)\right|_{\left[0,1 / \iota_{n}\right]} \in \mathfrak{D}_{\left[0,1 / \iota_{n}\right]}^{k} \quad \text { with } \quad \mu_{\tau_{n}}:[0,1 / \ell] \ni t \mapsto \mu\left(t \cdot \tau_{n}\right) .
$$

- We define $t_{n, m}:=m / \iota_{n}$ for $m=0, \ldots, \iota_{n}$; as well as $\phi_{n}[m]:\left[t_{n, m}, t_{n, m+1}\right] \ni t \mapsto \chi_{n}\left(\cdot-t_{n, m}\right) \in \mathfrak{g}$ for $m=0, \ldots, \iota_{n-1}$. Then, we have 14

$$
\int \phi_{n}[m] \stackrel{d]}{=} \int \chi_{n}=\mu_{\tau_{n}}\left(1 / \iota_{n}\right)=\mu\left(\tau_{n} / \iota_{n}\right) \quad \forall m=0, \ldots, \iota_{n-1} .
$$

- We define $\phi_{n} \in \mathfrak{D P} \mathrm{P}^{0}([0,1], \mathfrak{g})$ by

$$
\begin{array}{ll}
\left.\phi_{n}\right|_{\left[t_{n, m}, t_{n, m+1}\right)}:=\left.\phi_{n}[m]\right|_{\left[t_{n, m}, t_{n, m+1}\right)} & \forall m=0, \ldots, \iota(n)-2, \\
\left.\phi_{n}\right|_{\left[t_{\left.n, \iota_{n-1}, t_{n, \iota_{n}}\right]}:=\phi_{n}\left[\iota_{n}-1\right] ;\right.}
\end{array}
$$

and obtain

$$
\int \phi_{n} \stackrel{(27)}{=} \int_{t_{n, \iota_{n}-1}}^{t_{n, \iota_{n}}} \phi_{n}\left[\iota_{n}-1\right] \cdot \ldots \cdot \int_{t_{n, 0}}^{t_{n, 1}} \phi_{n}[0] \stackrel{(44)}{=} \mu\left(\tau_{n} / \iota_{n}\right)^{\iota_{n}} \quad \forall n \in \mathbb{N} .
$$

Then, for each $n \in \mathbb{N}$ and $\mathfrak{p} \in \mathfrak{P}$, we have

$$
\begin{aligned}
\cdot \mathfrak{p}_{\infty}\left(\phi_{n}-\tau \cdot \phi_{X}\right) & \leq \mathfrak{p}_{\infty}\left(\phi_{n}-\tau_{n} \cdot X\right)+\mathfrak{p}\left(\tau_{n} \cdot X-\tau \cdot X\right) \\
& =\mathfrak{p}_{\infty}\left(\left.\delta^{r}\left(\mu_{\tau_{n}}\right)\right|_{\left[0,1 / \iota_{n}\right]}-\tau_{n} \cdot X\right)+\left|\tau-\tau_{n}\right| \cdot \mathfrak{p}(X) \\
& \stackrel{400}{\leq} \ell \cdot \sup \left\{\cdot \mathfrak{p}\left(\phi\left(\tau_{n} \cdot t\right)-\phi(0)\right) \mid t \in\left[0,1 / \iota_{n}\right]\right\}+\left|\tau-\tau_{n}\right| \cdot \mathfrak{p}(X) .
\end{aligned}
$$

\footnotetext{
$\overline{{ }^{14} \text { In the first step below, d) } \text { is applied with } \varrho:}\left[t_{n, m}, t_{n, m+1}\right] \mapsto t-t_{n, m} \in\left[0,1 / \iota_{n}\right]$.
} 
For the case that $\phi \in C^{\text {lip }}([0,1], \mathfrak{g})$ holds, we furthermore obtain

$$
\begin{aligned}
\mathfrak{p}_{\infty}\left(\phi_{n}-\tau \cdot \phi_{X}\right) \stackrel{\text { (46), (41) }}{\leq} \ell^{2} / \iota_{n} \cdot \operatorname{Lip}(\mathfrak{p}, \phi)+\left|\tau-\tau_{n}\right| \cdot \mathfrak{p}(X) \\
\leq \underbrace{(\operatorname{Lip}(\cdot \mathfrak{p}, \phi)+\mathfrak{p}(X))}_{\mathfrak{c}_{\mathfrak{p}}} \cdot \underbrace{\left(\ell^{2} / \iota_{n}+\left|\tau-\tau_{n}\right|\right)}_{\lambda_{n}}
\end{aligned}
$$

for each $n \in \mathbb{N}$ and $\mathfrak{p} \in \mathfrak{P}$; whereby $\lim _{n} \lambda_{n}=0$ holds. We thus have

$$
\begin{array}{ll} 
& \mathfrak{D P}^{0}([0,1], \mathfrak{g}) \supseteq\left\{\phi_{n}\right\}_{n \in \mathbb{N}} \rightarrow_{\mathfrak{s}} \tau \cdot \phi_{X} \\
\text { as well as } \quad & \mathfrak{D P}^{0}([0,1], \mathfrak{g}) \supseteq\left\{\phi_{n}\right\}_{n \in \mathbb{N}} \rightarrow_{\mathfrak{m}} \tau \cdot \phi_{X} \quad \text { if } \quad \phi \in C^{\text {lip }}([0,1], \mathfrak{g}) \quad \text { holds. }
\end{array}
$$

In both cases, by Lemma 17, there exists some $\mathbb{N} \ni n_{V}^{\prime} \geq n_{V}$ with

$$
\left[\left.\int \tau \cdot \phi_{X}\right|_{[0,1]}\right]^{-1} \cdot\left[\int \phi_{n}\right] \in V \quad \forall n \geq n_{V}^{\prime}
$$

and we obtain for $n \geq n_{V}^{\prime}$ that

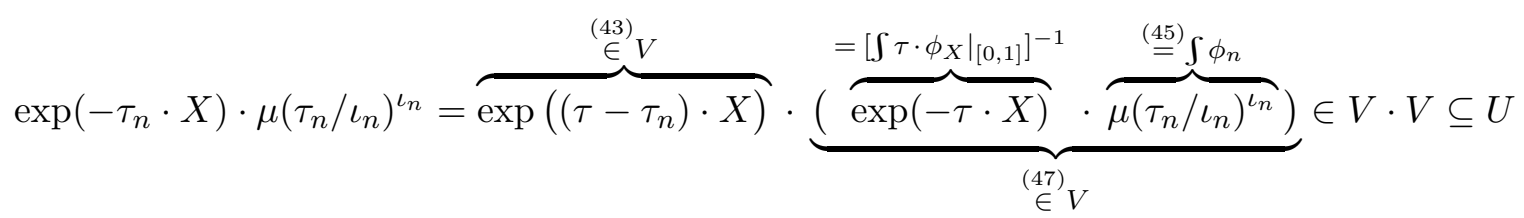

holds, which contradicts (42).

\section{Differentiation}

In this section, we discuss several differentiability properties of the evolution map. The whole discussion is based on the following generalization of Proposition 7 in [7].

Proposition 2. Let $\left\{\varepsilon_{n}\right\}_{n \in \mathbb{N}} \subseteq C^{0}\left(\left[r, r^{\prime}\right], \mathfrak{g}\right), \chi \in C^{0}\left(\left[r, r^{\prime}\right], \mathfrak{g}\right)$, and $\mathbb{R}_{\neq 0} \supseteq\left\{h_{n}\right\}_{n \in \mathbb{N}} \rightarrow 0$ be given with $\left\{h_{n} \cdot \chi+h_{n} \cdot \varepsilon_{n}\right\}_{n \in \mathbb{N}} \subseteq \mathfrak{D}_{\left[r, r^{\prime}\right]}$, such that

i) $\lim _{n} \varepsilon_{n}(t)=0 \quad$ holds for each $t \in\left[r, r^{\prime}\right]$,

ii) $\left.\sup \left\{\mathfrak{p}_{\infty}\left(\varepsilon_{n}\right) \mid n \in \mathbb{N}\right)\right\}<\infty \quad$ holds for each $\mathfrak{p} \in \mathfrak{P}$.

Then, the following two conditions are equivalent:

a) $\lim _{n}^{\infty} \Xi\left(\int_{r}^{\bullet} h_{n} \cdot \chi+h_{n} \cdot \varepsilon_{n}\right)=0$.

b) $\lim _{n}^{\infty} 1 / h_{n} \cdot \Xi\left(\int_{r}^{\bullet} h_{n} \cdot \chi+h_{n} \cdot \varepsilon_{n}\right)=\int_{r}^{\bullet}\left(\mathrm{d}_{e} \Xi \circ \chi\right)(s) \mathrm{d} s \in \bar{E}$.

The proof of Proposition 2 will be established in Sect. 6.4. We now first use this proposition, to discuss the differential of the evolution maps as well as the differentiation of parameter-dependent integrals.

\subsection{Some Technical Statements}

We will need the following variation of Proposition 2 ;

Corollary 2. Let $\delta>0,\left\{\varepsilon_{h}\right\}_{h \in \mathcal{D}_{\delta}} \subseteq C^{0}\left(\left[r, r^{\prime}\right], \mathfrak{g}\right)$, and $\chi \in C^{0}\left(\left[r, r^{\prime}\right], \mathfrak{g}\right)$ be given with $\{h \cdot \chi+h$. $\left.\varepsilon_{h}\right\}_{h \in \mathcal{D}_{\delta}} \subseteq \mathfrak{D}_{\left[r, r^{\prime}\right]}$, such that
i) $\lim _{h \rightarrow 0} \varepsilon_{h}(t)=0$
holds for each $t \in\left[r, r^{\prime}\right]$,
ii) $\sup \left\{\mathfrak{p}_{\infty}\left(\varepsilon_{h}\right) \mid h \in \mathcal{D}_{\delta_{\mathfrak{p}}}\right\}<\infty$
holds for some $0<\delta_{\mathfrak{p}} \leq \delta$, for each $\mathfrak{p} \in \mathfrak{P}$. 
Then, the following conditions are equivalent 15

a) $\lim _{h \rightarrow 0}^{\infty} \Xi\left(\int_{r}^{\bullet} h \cdot \chi+h \cdot \varepsilon_{h}\right)=0$.

b) $\lim _{n}^{\infty} \Xi\left(\int_{r}^{\bullet} h_{n} \cdot \chi+h_{n} \cdot \varepsilon_{h_{n}}\right)=0$ for each sequence $\mathcal{D}_{\delta} \supseteq\left\{h_{n}\right\}_{n \in \mathbb{N}} \rightarrow 0$.

c) $\lim _{n}^{\infty} 1 / h_{n} \cdot \Xi\left(\int_{r}^{\bullet} h_{n} \cdot \chi+h_{n} \cdot \varepsilon_{h_{n}}\right)=\int_{r}^{\bullet}\left(\mathrm{d}_{e} \Xi \circ \chi\right)(s) \mathrm{d} s \in \bar{E}$ for each sequence $\mathcal{D}_{\delta} \supseteq\left\{h_{n}\right\}_{n \in \mathbb{N}} \rightarrow 0$.

d) $\left.\frac{\mathrm{d}}{\mathrm{d} h}\right|_{h=0} ^{\infty} \Xi\left(\int_{r}^{\bullet} h \cdot \chi+h \cdot \varepsilon_{h}\right)=\int_{r}^{\bullet}\left(\mathrm{d}_{e} \Xi \circ \chi\right)(s) \mathrm{d} s \in \bar{E}$.

Proof. By Lemma 1 (applied to $(G,+) \equiv(\bar{E},+)$ there), a) is equivalent to b). Moreover, by Proposition 2, b) is equivalent to c), because

- Condition i) implies Condition i) in Proposition 2, for $\varepsilon_{n} \equiv \varepsilon_{h_{n}}$ there,

- Condition ii) implies Condition ii) in Proposition 2, for $\varepsilon_{n} \equiv \varepsilon_{h_{n}}$ there.

Finally, by Lemma 1 (applied to $(G,+) \equiv(\bar{E},+)$ there), c) is equivalent to d),

Given a net $\left\{\psi_{\alpha}\right\}_{\alpha \in I} \subseteq C^{0}\left(\left[r, r^{\prime}\right], \mathfrak{g}\right)$, and some $\psi \in C^{0}\left(\left[r, r^{\prime}\right], \mathfrak{g}\right)$, we write $\left\{\psi_{\alpha}\right\}_{\alpha \in I} \rightarrow_{\mathfrak{n} .0} \psi$ if

$$
\lim _{\alpha} \mathfrak{p}\left(\psi-\psi_{\alpha}\right)=0 \quad \forall \mathfrak{p} \in \mathfrak{P} .
$$

Lemma 18. Suppose that $G$ is Mackey k-continuous for $k \in \mathbb{N} \sqcup\{$ lip, $\infty, \mathrm{c}\}$. Suppose furthermore that we are given $\mathfrak{D}_{\left[r, r^{\prime}\right]}^{k} \supseteq\left\{\phi_{n}\right\}_{n \in \mathbb{N}} \rightarrow_{\text {m.k }} \phi \in \mathfrak{D}_{\left[r, r^{\prime}\right]}^{k}$ as well as $\mathfrak{D}_{\left[r, r^{\prime}\right]}^{k} \supseteq\left\{\psi_{\alpha}\right\}_{\alpha \in I} \rightarrow_{\mathfrak{n} .0} \psi \in \mathfrak{D}_{\left[r, r^{\prime}\right]}^{k}$ for $\left[r, r^{\prime}\right] \in \mathfrak{K}$, such that the expressions

$$
\begin{aligned}
\xi(\phi, \psi) & :=\mathrm{d}_{e} \mathrm{~L}_{\boldsymbol{}}\left(\int \operatorname{Ad}_{\left[\int_{r}^{s} \phi\right]^{-1}}(\psi(s)) \mathrm{d} s\right) \\
\xi\left(\phi_{n}, \psi_{\alpha}\right) & :=\mathrm{d}_{e} \mathrm{~L}_{\int \phi_{n}}\left(\int \operatorname{Ad}_{\left[\int_{r}^{s} \phi_{n}\right]^{-1}}\left(\psi_{\alpha}(s)\right) \mathrm{d} s\right) \quad \forall n \in \mathbb{N}
\end{aligned}
$$

are well defined; i.e., such that the occurring Riemann integrals exist in $\mathfrak{g}$. Then, we have

$$
\lim _{(n, \alpha)} \xi\left(\phi_{n}, \psi_{\alpha}\right)=\xi(\phi, \psi)
$$

Proof. This follows by the same arguments as in Corollary 13 and Lemma 41 in [7. For completeness, the adapted argumentation is provided in Appendix A.4.

\subsection{The Differential of the Evolution Map}

We now discuss the differential of the evolution map - for which we recall the conventions fixed in Remark 3. Then, Corollary 2 (with $\varepsilon_{h} \equiv 0$ there) provides us with

Proposition 3. Suppose that $(\phi, \psi)$ is admissible, with $\operatorname{dom}[\phi], \operatorname{dom}[\psi]=\left[r, r^{\prime}\right]$.

1) The pair $(\phi, \psi)$ is regular if and only if we have

$$
\left.\frac{\mathrm{d}}{\mathrm{d} h}\right|_{h=0} ^{\infty} \Xi\left(\left[\int_{r}^{\bullet} \phi\right]^{-1}\left[\int_{r}^{\bullet} \phi+h \cdot \psi\right]\right)=\int_{r}^{\bullet}\left(\mathrm{d}_{e} \Xi \circ \operatorname{Ad}_{\left[\int_{r}^{s} \phi\right]^{-1}}\right)(\psi(s)) \mathrm{d} s \in \bar{E} .
$$

2) If $(\phi, \psi)$ is regular, then $(-\delta, \delta) \ni h \mapsto \int \phi+h \cdot \psi \in G$ is differentiable at $h=0$ (for $\delta>0$ suitably small) if and only if $\int \operatorname{Ad}_{\left[\int_{r}^{s} \phi\right]^{-1}}(\psi(s)) \mathrm{d} s \in \mathfrak{g}$ holds. In this case, we have

$$
\left.\frac{\mathrm{d}}{\mathrm{d} h}\right|_{h=0} \int \phi+h \cdot \psi=\mathrm{d}_{e} \mathrm{~L}_{\int \phi}\left(\int \operatorname{Ad}_{\left[\int_{r}^{s} \phi\right]^{-1}}(\psi(s)) \mathrm{d} s\right) .
$$

\footnotetext{
${ }^{15}$ Recall Remark 2 for the notation used in $\mathrm{d}$ ).
} 
Proof. 1) For $|h|<\delta$, with $\delta>0$ suitably small, we have

$$
\Xi\left(\left[\int_{r}^{t} \phi\right]^{-1}\left[\int_{r}^{t} \phi+h \cdot \psi\right]\right) \stackrel{b b}{=} \Xi(\int_{r}^{t} h \cdot \overbrace{\operatorname{Ad}_{\left[\int_{r}^{\bullet} \phi\right]^{-1}}(\psi)}^{=: \chi}) \quad \forall t \in\left[r, r^{\prime}\right] .
$$

We obtain from the Equivalence of a) and d) in Corollary 2 for $\varepsilon_{h} \equiv 0$ there that (third step)

$$
\begin{aligned}
& \lim _{h \rightarrow 0}^{\infty} \int_{r}^{\bullet} \phi+h \cdot \psi=\int_{r}^{\bullet} \phi \\
& \Longleftrightarrow \quad \lim _{h \rightarrow 0}^{\infty} \Xi\left(\left[\int_{r}^{\bullet} \phi\right]^{-1}\left[\int_{r}^{\bullet} \phi+h \cdot \psi\right]\right)=0 \\
& \stackrel{499)}{\Longrightarrow} \quad \lim _{h \rightarrow 0}^{\infty} \Xi\left(\int_{r}^{\bullet} h \cdot \chi\right)=0 \\
& \left.\Longleftrightarrow \quad \frac{\mathrm{d}}{\mathrm{d} h}\right|_{h=0} ^{\infty} \Xi\left(\int_{r}^{\bullet} h \cdot \chi\right)=\int_{r}^{\bullet}\left(\mathrm{d}_{e} \Xi \circ \chi\right)(s) \mathrm{d} s \in \bar{E} \\
& \left.\stackrel{\mathrm{d}}{\Longrightarrow}\right|_{h=0} ^{\infty} \Xi\left(\left[\int_{r}^{\bullet} \phi\right]^{-1}\left[\int_{r}^{\bullet} \phi+h \cdot \psi\right]\right)=\int_{r}^{\bullet}\left(\mathrm{d}_{e} \Xi \circ \operatorname{Ad}_{\left[\int_{r}^{s} \phi\right]^{-1}}\right)(\psi(s)) \mathrm{d} s \in \bar{E} .
\end{aligned}
$$

2) Let $(\phi, \psi)$ be regular; and $\mu:(-\delta, \delta) \ni h \mapsto \int \phi+h \cdot \psi \in G$.

- Suppose that $\int \operatorname{Ad}_{\left[\int_{r}^{s} \phi\right]^{-1}}(\psi(s)) \mathrm{d} s \in \mathfrak{g}$ holds; and let (shrink $\delta>0$ if necessary)

$$
\gamma:(-\delta, \delta) \rightarrow \mathcal{V}, \quad h \mapsto \Xi\left(\left[\int \phi\right]^{-1}\left[\int \phi+h \cdot \psi\right]\right) .
$$

Then, we have

$$
\dot{\gamma}(0) \stackrel{\text { Part } 1)}{=} \int\left(\mathrm{d}_{e} \Xi \circ \operatorname{Ad}_{\left[\int_{r}^{s} \phi\right]^{-1}}\right)(\psi(s)) \mathrm{d} s \stackrel{(11)}{=} \mathrm{d}_{e} \Xi\left(\int \operatorname{Ad}_{\left[\int_{r}^{s} \phi\right]^{-1}}(\psi(s)) \mathrm{d} s\right) .
$$

Since $\mu=\Xi^{\prime-1} \circ \gamma\left(\right.$ thus, $\gamma=\Xi^{\prime} \circ \mu$ ) holds for the chart

$$
\Xi^{\prime}: \int \phi \cdot \mathcal{U}=: \mathfrak{U}^{\prime} \rightarrow \mathcal{V}, \quad g \mapsto \Xi\left(\left[\int \phi\right]^{-1} \cdot g\right),
$$

(50) shows that $\mu$ is differentiable at 0 - Specifically, we have, cf. Remark 3

$$
\begin{aligned}
\dot{\mu}(0) & =\mathrm{d}_{0} \Xi^{\prime-1}\left(\left.\frac{\mathrm{d}}{\mathrm{d} h}\right|_{h=0}\left(\Xi^{\prime} \circ \mu\right)(h)\right) \\
& \stackrel{(500)}{=}\left(\mathrm{d}_{0} \Xi^{\prime-1} \circ \mathrm{d}_{e} \Xi\right)\left(\int \operatorname{Ad}_{\left[\int_{r}^{s} \phi\right]^{-1}}(\psi(s)) \mathrm{d} s\right) \\
& =\left(\mathrm{d}_{e} \mathrm{~L}_{\int \phi} \circ \mathrm{d}_{0} \Xi^{-1} \circ \mathrm{d}_{e} \Xi\right)\left(\int \operatorname{Ad}_{\left[\int_{r}^{s} \phi\right]^{-1}}(\psi(s)) \mathrm{d} s\right) \\
& =\mathrm{d}_{e} \mathrm{~L}_{\int \phi}\left(\int \operatorname{Ad}_{\left[\int_{r}^{s} \phi\right]^{-1}}(\psi(s)) \mathrm{d} s\right) ;
\end{aligned}
$$

which shows (48).

- Suppose that $\mu$ is differentiable at $h=0$. Then, for $\Xi^{\prime}$ as in (51) we have, cf. Remark 3

$$
\left.E \ni \frac{\mathrm{d}}{\mathrm{d} h}\right|_{h=0}\left(\Xi^{\prime} \circ \mu\right)(h)=\left.\frac{\mathrm{d}}{\mathrm{d} h}\right|_{h=0} \Xi\left(\left[\int \phi\right]^{-1}\left[\int \phi+h \cdot \psi\right]\right) \stackrel{\operatorname{Part} 1)]}{=} \int\left(\mathrm{d}_{e} \Xi \circ \operatorname{Ad}_{\left[\int_{r}^{s} \phi\right]^{-1}}\right)(\psi(s)) \mathrm{d} s .
$$

We obtain

$$
\mathfrak{g} \ni \mathrm{d}_{0} \Xi^{-1}\left(\int\left(\mathrm{d}_{e} \Xi \circ \operatorname{Ad}_{\left[\int_{r}^{s} \phi\right]^{-1}}\right)(\psi(s)) \mathrm{d} s\right) \stackrel{(11)}{=} \int \operatorname{Ad}_{\left[\int_{r}^{s} \phi\right]^{-1}}(\psi(s)) \mathrm{d} s .
$$

In particular, (48) holds by the previous point. 


\subsubsection{The Generic Case}

Combining Proposition 3 with Theorem 1 and Lemma 15, we obtain

Theorem 2. Suppose that $G$ is $C^{k}$-semiregular for $k \in \mathbb{N} \sqcup\{\operatorname{lip}, \infty\}$. Then, evol $_{\mathrm{k}}$ is differentiable if and only if $\mathfrak{g}$ is $\mathrm{k}$-complete. In this case, $\operatorname{evol}_{\left[r, r^{\prime}\right]}^{k}$ is differentiable for each $\left[r, r^{\prime}\right] \in \mathfrak{K}$, with

$$
\mathrm{d}_{\phi} \operatorname{evol}_{\left[r, r^{\prime}\right]}^{k}(\psi)=\mathrm{d}_{e} \mathrm{~L}_{\int \phi}\left(\int \operatorname{Ad}_{\left[\int_{r}^{s} \phi\right]^{-1}}(\psi(s)) \mathrm{d} s\right) \quad \forall \phi, \psi \in C^{k}\left(\left[r, r^{\prime}\right], \mathfrak{g}\right) .
$$

In particular,

a) $\mathrm{d}_{\phi} \operatorname{evol}_{\left[r, r^{\prime}\right]}^{k}: C^{k}\left(\left[r, r^{\prime}\right], \mathfrak{g}\right) \rightarrow T_{\int_{\phi}} G$ is linear and $C^{0}$-continuous for each $\phi \in C^{k}\left(\left[r, r^{\prime}\right], \mathfrak{g}\right)$,

b) for each sequence $\left\{\phi_{n}\right\}_{n \in \mathbb{N}} \rightarrow_{\mathfrak{m} . \mathrm{k}} \phi$, and each net $\left\{\psi_{\alpha}\right\}_{\alpha \in I} \rightarrow_{\mathfrak{n} .0} \psi$, we have

$$
\lim _{(n, \alpha)} \mathrm{d}_{\phi_{n}} \operatorname{evol}_{\left[r, r^{\prime}\right]}^{k}\left(\psi_{\alpha}\right)=\mathrm{d}_{\phi} \operatorname{evol}_{\left[r, r^{\prime}\right]}^{k}(\psi) .
$$

Proof. The first part is clear from Theorem 1, Lemma 15, Remark 4, and Proposition 3 2), Then, b) is clear from Lemma [18. Moreover, (by the first part) $d_{\phi} \operatorname{evol}_{\left[r, r^{\prime}\right]}^{k}$ is linear; with (cf. (3))

$$
\mathrm{d}_{\phi} \operatorname{evol}_{\left[r, r^{\prime}\right]}^{k}(\psi)=\mathrm{d}_{\left(\int \phi, e\right)} \mathrm{m}\left(0, \Gamma_{\phi}(\psi)\right) \quad \text { for } \quad \Gamma_{\phi}: C^{k}([0,1], \mathfrak{g}) \ni \psi \rightarrow \int \operatorname{Ad}_{\left[\int_{r}^{s} \phi\right]^{-1}}(\psi(s)) \mathrm{d} s \in \mathfrak{g} .
$$

Then, since $\Gamma_{\phi}$ is $C^{0}$-continuous by (9) and I), a) is clear from smoothness of the Lie group multiplication.

Corollary 3. Suppose that $G$ is $C^{k}$-semiregular for $k \in \mathbb{N} \sqcup\{$ lip, $\infty\}$, and that $\mathfrak{g}$ is $\mathrm{k}$-complete. Then, $\mu: \mathbb{R} \ni h \mapsto \int \phi+h \cdot \psi$ is of class $C^{1}$ for each $\phi, \psi \in C^{k}\left(\left[r, r^{\prime}\right], \mathfrak{g}\right)$ and $\left[r, r^{\prime}\right] \in \mathfrak{K}$.

Proof. Theorem 1 and Lemma 15] show that $\mu$ is continuous. Moreover, for each $t \in \mathbb{R}$, and each sequence $\left\{h_{n}\right\}_{n \in \mathbb{N}} \rightarrow 0$, we have $\left\{\phi+\left(t+h_{n}\right) \cdot \psi\right\}_{n \in \mathbb{N}} \rightarrow_{\mathfrak{m} . \mathrm{k}}(\phi+t \cdot \psi)$; thus,

$$
\lim _{n} \dot{\mu}\left(t+h_{n}\right)=\lim _{n} \mathrm{~d}_{\phi+\left(t+h_{n}\right) \cdot \psi} \operatorname{evol}_{\left[r, r^{\prime}\right]}^{k}(\psi)=\mathrm{d}_{\phi+t \cdot \psi} \operatorname{evol}_{\left[r, r^{\prime}\right]}^{k}(\psi)=\dot{\mu}(t)
$$

by Theorem $2[b]$, This shows that $\dot{\mu}$ is continuous, i.e., that $\mu$ is of class $C^{1}$.

\section{Remark 8.}

1) It is straightforward from Corollary [3, the differentiation rules $\mathbf{d}$ ) and $\mathbf{c}$ ), as well as (3), (12), c), and e) (for $\Psi \equiv \operatorname{Conj}_{g}$ there) that (48) also holds for all $\phi, \psi \in \mathfrak{D P}^{k}\left(\left[r, r^{\prime}\right], \mathfrak{g}\right)$, for each $\left[r, r^{\prime}\right] \in \mathfrak{K}$.

2) Expectably, $\mu$ as defined in Corollary 3 is even of class $C^{\infty}$. A detailed proof of this fact, however, would require further technical preparation - which we do not want to carry out at this point.

3) Expectably, the equivalence

$$
\lim _{h \rightarrow 0}^{\infty} \Xi\left(\int_{r}^{\bullet} h \cdot \chi\right)=\left.0 \quad \Longleftrightarrow \quad \frac{\mathrm{d}}{\mathrm{d} h}\right|_{h=0} ^{\infty} \Xi\left(\int_{r}^{\bullet} h \cdot \chi\right)=\int_{r}^{\bullet}\left(\mathrm{d}_{e} \Xi \circ \chi\right)(s) \mathrm{d} s \in \bar{E}
$$

also holds for $\chi \in \mathfrak{D P}^{0}\left(\left[r, r^{\prime}\right], \mathfrak{g}\right)$ - implying that Proposition [3] 1) carries over to the piecewise category. This might be shown by the same arguments (Taylor expansion) as used in the proof of Lemma 7 in [7] (cf. Lemma [3) additionally using (27) as well as that for $n \in \mathbb{N}$ fixed,

$$
f: G^{n} \rightarrow G, \quad\left(g_{1}, \ldots, g_{n}\right) \mapsto g_{1} \cdot \ldots \cdot g_{n}
$$

is smooth, with $\mathrm{d}_{(e, \ldots, e)} f\left(X_{1}, \ldots, X_{n}\right)=X_{1}+\cdots+X_{n}$ for all $X_{1}, \ldots, X_{n} \in \mathfrak{g}$. The details, however, appear to be quite technical, so that we leave this issue to another paper. 


\subsubsection{The Exponential Map}

We recall the conventions fixed in Sect. 2.2.3, specifically that exp $=\left.\operatorname{evol}_{[0,1]}^{c} \circ \mathfrak{i}\right|_{\operatorname{dom}[\exp ]}$ holds. Then, Proposition [3] 2 , for $k \equiv \mathrm{c}$ and $\left[r, r^{\prime}\right] \equiv[0,1]$ there, reads as follows.

Corollary 4. Suppose that $(\mathfrak{i}(X), \mathfrak{i}(Y))$ is regular for $X, Y \in \mathfrak{g}$. Then, $(-\delta, \delta) \ni h \mapsto \exp (X+h \cdot Y)$ is differentiable at $h=0$ (for $\delta>0$ suitably small) if and only if $\int \operatorname{Ad}_{\exp (-s \cdot X)}(Y) \mathrm{d} s \in \mathfrak{g}$ holds. In this case, we have

$$
\left.\frac{\mathrm{d}}{\mathrm{d} h}\right|_{h=0} \exp (X+h \cdot Y)=\mathrm{d}_{e} \mathrm{~L}_{\exp (X)}\left(\int \operatorname{Ad}_{\exp (-s \cdot X)}(Y) \mathrm{d} s\right) .
$$

\section{Remark 9.}

1) Suppose that $G$ admits an exponential map; and that $G$ is weakly c-continuous. Then, Corollary 4 shows that we have

$$
\left.\frac{\mathrm{d}}{\mathrm{d} h}\right|_{h=0} \exp (X+h \cdot Y)=\mathrm{d}_{e} \mathrm{~L}_{\exp (X)}\left(\int \operatorname{Ad}_{\exp (-s \cdot X)}(Y) \mathrm{d} s\right) \quad \forall X, Y \in \mathfrak{g}
$$

if and only if $\mathfrak{g}$ is c-complete. For instance, $G$ is weakly c-continuous, and $\mathfrak{g}$ is c-complete if

- $\exp : \mathfrak{g} \rightarrow G$ is of class $C^{1}$, by Remark 7, Remark 7, [), and Corollary 4.

- $G$ is abelian, by Corollary 1 and Remark 4 .

2) Suppose that $\mathfrak{g}$ is c-complete; and that $G$ admits a continuous exponential map. Then, $G$ is $C^{\mathrm{c}}$ semiregular; and $G$ is weakly c-continuous by Remark 7 1) and Remark 75). More formally, (52) then reads

$$
\mathrm{d}_{\phi} \operatorname{evol}_{\mathrm{c}}(\psi)=\mathrm{d}_{e} \mathrm{~L}_{\int \phi}\left(\int \operatorname{Ad}_{\left[\int_{r}^{s} \psi\right]^{-1}}(\psi(s)) \mathrm{d} s\right) \quad \forall \phi, \psi \in C^{\mathrm{c}}([0,1], \mathfrak{g}) .
$$

The same arguments as in [7] then show that evol $_{\mathrm{c}}$ (thus $\exp$ ) is of class $C^{1}$. More specifically, one has to replace Lemma 23 by Lemma 10 in the proof of Lemma 41 in [7]. Then, substituting Equation (95) in [7] by (53), the proof of Corollary 13 in [7] just carries over to the case where $k=\mathrm{c}$ holds (a similar adaption has been done in the proof of Lemma 18).

As in the Lipschitz case, cf. Remark 7 in [7], it is to be expected that a (quite elaborate and technical) induction shows that $\exp$ is even smooth if $\mathfrak{g}$ is Mackey complete (or, more generally, if all the occurring iterated Riemann integrals exist in $\mathfrak{g})$.

\subsection{Integrals with Parameters}

Given an open interval $J \subseteq \mathbb{R}$ as well as $x \in J$, in the following, we denote

$$
J[x]:=\left\{h \in \mathbb{R}_{\neq 0} \mid x+h \in J\right\} .
$$

The next theorem generalizes Theorem 5 in [7] (with significantly simplified proof).

Theorem 3. Suppose that $G$ is Mackey k-continuous for $k \in \mathbb{N} \sqcup\{$ lip, $\infty, c\}$ - additionally abelian if $k=\mathrm{c}$ holds. Let $\Phi: I \times\left[r, r^{\prime}\right] \rightarrow \mathfrak{g}\left(I \subseteq \mathbb{R}\right.$ open) be given with $\Phi(z, \cdot) \in \mathfrak{D}_{\left[r, r^{\prime}\right]}^{k}$ for each $z \in I$. Then,

$$
\left.\frac{\mathrm{d}}{\mathrm{d} h}\right|_{h=0} ^{\infty} \Xi\left(\left[\int_{r}^{\bullet} \Phi(x, \cdot)\right]^{-1}\left[\int_{r}^{\bullet} \Phi(x+h, \cdot)\right]\right)=\int_{r}^{\bullet}\left(\mathrm{d}_{e} \Xi \circ \operatorname{Ad}_{\left[\int_{r}^{s} \Phi(x, \cdot)\right]^{-1}}\right)\left(\partial_{z} \Phi(x, s)\right) \mathrm{d} s \in \bar{E}
$$

holds for $x \in I$, provided that

a) We have $\left(\partial_{z} \Phi\right)(x, \cdot) \in C^{k}\left(\left[r, r^{\prime}\right], \mathfrak{g}\right) 16$

\footnotetext{
$\overline{{ }^{16} \text { More specifically, this means that for each } t} \in\left[r, r^{\prime}\right]$, the map $I \ni z \mapsto \Phi(z, t)$ is differentiable at $z=x$ with derivative $\left(\partial_{z} \Phi\right)(x, t)$, such that $\left(\partial_{z} \Phi\right)(x, \cdot) \in C^{k}\left(\left[r, r^{\prime}\right], \mathfrak{g}\right)$ holds. The latter condition in particular ensures that $\mathfrak{. p}_{\infty}^{\mathrm{s}}\left(\left(\partial_{z} \Phi\right)(x, \cdot)\right)<\infty$ holds for each $\mathfrak{p} \in \mathfrak{P}$ and $\mathrm{s} \preceq k$, cf. ii)
} 
b) For each $\mathfrak{p} \in \mathfrak{P}$ and $\mathrm{s} \preceq k$, there exists $L_{\mathfrak{p}, \mathrm{s}} \geq 0$, as well as $I_{\mathfrak{p}, \mathrm{s}} \subseteq I$ open with $x \in I_{\mathfrak{p}, \mathrm{s}}$, such that

$$
1 /|h| \cdot \mathfrak{p}_{\infty}^{\mathrm{s}}(\Phi(x+h, \cdot)-\Phi(x, \cdot)) \leq L_{\mathfrak{p}, \mathrm{s}} \quad \forall h \in I_{\mathfrak{p}, \mathrm{s}}[x] .
$$

In particular, we have

$$
\left.\frac{\mathrm{d}}{\mathrm{d} h}\right|_{h=0} \int \Phi(x+h, \cdot)=\mathrm{d}_{e} \mathrm{~L}_{\int \Phi(x, \cdot)}\left(\int \operatorname{Ad}_{\left[\int_{r}^{s} \Phi(x, \cdot)\right]^{-1}}\left(\partial_{z} \Phi(x, s)\right) \mathrm{d} s\right)
$$

if and only if the Riemann integral on the right side exists in $\mathfrak{g}$.

Proof. The last statement follows from the first statement and Lemma 3 - just as in the proof of Proposition [3]2). Now, for $x+h \in I$, we have

$$
\Phi(x+h, t)=\Phi(x, t)+h \cdot \partial_{z} \Phi(x, t)+h \cdot \varepsilon(x+h, t) \quad \forall t \in\left[r, r^{\prime}\right],
$$

with $\varepsilon: I \times\left[r, r^{\prime}\right] \rightarrow \mathfrak{g}$ such that

i) $\lim _{h \rightarrow 0} \varepsilon(x+h, t)=\varepsilon(x, t)=0 \quad \forall t \in\left[r, r^{\prime}\right]$,

ii) $\cdot \mathfrak{p}_{\infty}^{\mathrm{s}}(\varepsilon(x+h, \cdot)) \leq L_{\mathfrak{p}, \mathrm{s}}+\cdot \mathfrak{p}_{\infty}^{\mathrm{s}}\left(\left(\partial_{z} \Phi\right)(x, \cdot)\right)=: C_{\mathfrak{p}, \mathrm{s}}<\infty \quad \forall h \in I_{\mathfrak{p}, \mathrm{s}}[x] \quad$ for all $\mathfrak{p} \in \mathfrak{P}, \mathrm{s} \preceq k$.

We let $\alpha:=\int_{r}^{\bullet} \Phi(x, \cdot)$; and obtain

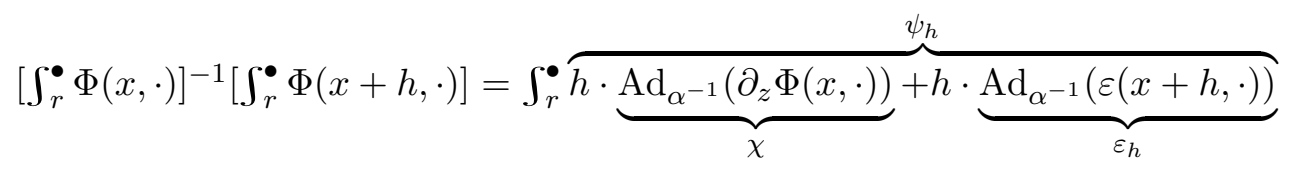

with $\psi_{h} \in \mathfrak{D}_{\left[r, r^{\prime}\right]}^{k}$, because our presumptions ensure that $\chi, \varepsilon_{h} \in C^{k}\left(\left[r, r^{\prime}\right], \mathfrak{g}\right)$ holds. By Lemma 6 and Lemma 7, for each $\mathfrak{p} \in \mathfrak{P}$ and $\mathrm{s} \preceq k$, there exists some $\mathfrak{p} \leq \mathfrak{q} \in \mathfrak{P}$ with 17

$$
\cdot \mathfrak{p}_{\infty}^{\mathrm{s}}\left(\psi_{h}\right) \leq|h| \cdot \mathfrak{q}_{\infty}^{\mathrm{s}}\left(\partial_{z} \Phi(x, \cdot)+\varepsilon(x+h, \cdot)\right) \underset{b}{\leq}|h| \cdot L_{\mathfrak{q}, \mathrm{s}} \quad \forall h \in I_{\mathfrak{q}, \mathrm{s}}[x] .
$$

For each fixed sequence $I[x] \supseteq\left\{h_{n}\right\}_{n \in \mathbb{N}} \rightarrow 0$, we thus have $\psi_{h_{n}} \rightarrow_{\mathfrak{m} . \mathrm{k}} 0$. Since $G$ is Mackey $\mathrm{k}$-continuous, this implies

$$
\lim _{n}^{\infty} \Xi\left(\int_{r}^{\bullet} h_{n} \cdot \chi+h_{n} \cdot \varepsilon_{h_{n}}\right)=\lim _{n}^{\infty} \Xi\left(\int_{r}^{\bullet} \psi_{h_{n}}\right)=0 .
$$

Now, for $\delta>0$ such small that $\mathcal{D}_{\delta} \subseteq I[x]$ holds, by i) and ii), $\left\{\varepsilon_{h}\right\}_{h \in \mathcal{D}_{\delta}}$ fulfills the presumptions in Corollary 2, We thus have

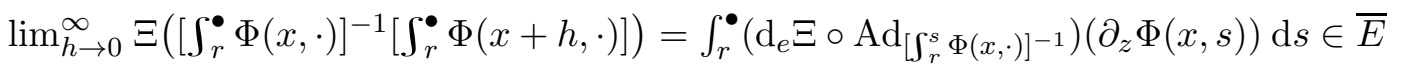

by (54), (55), as well as the equivalence of b) and d) in Corollary 2 ,

We immediately obtain

Corollary 5. Suppose that $G$ is $C^{k}$-semiregular for $k \in \mathbb{N} \sqcup\{$ lip, $\infty\}$; and that $\mathfrak{g}$ is $\mathrm{k}$-complete. Let $\Phi: I \times\left[r, r^{\prime}\right] \rightarrow \mathfrak{g}\left(I \subseteq \mathbb{R}\right.$ open) be given with $\Phi(z, \cdot) \in \mathfrak{D}_{\left[r, r^{\prime}\right]}^{k}$ for each $z \in I$. Then,

$$
\left.\frac{\mathrm{d}}{\mathrm{d} h}\right|_{h=0} \int \Phi(x+h, \cdot)=\mathrm{d}_{e} \mathrm{~L}_{\int \Phi(x, \cdot)}\left(\int \operatorname{Ad}_{\left[\int_{r}^{s} \Phi(x, \cdot)\right]^{-1}}\left(\partial_{z} \Phi(x, s)\right) \mathrm{d} s\right)
$$

holds for $x \in I$, provided that the conditions $a$ ) and $b$ ) in Theorem 3 are fulfilled.

Proof. This is clear from Theorem 1 and Theorem 3 ,

We furthermore obtain the following generalization of Corollary 11 in [7].

${ }^{17}$ If $k=\mathrm{c}$ holds, we can just choose $\mathrm{s}=0$ and $\mathfrak{q}=\mathfrak{p}$, because $G$ is presumed to be abelian in this case. 
Corollary 6. Suppose that $G$ is Mackey k-continuous for $k \in\{\infty, \mathrm{c}\}$-additionally abelian if $k=\mathrm{c}$ holds. Suppose furthermore that $\mathfrak{X}: I \rightarrow \operatorname{dom}[\exp ] \subseteq \mathfrak{g}$ is of class $C^{1}$; and define $\alpha:=\exp \circ \mathfrak{X}$. Then, for $x \in I$, we have

$$
\left.\frac{\mathrm{d}}{\mathrm{d} h}\right|_{h=0} \alpha(x+h)=\mathrm{d}_{e} \mathrm{~L}_{\exp (\mathfrak{X}(x))}\left(\int_{0}^{1} \operatorname{Ad}_{\exp (-s \cdot \mathfrak{X}(x))}(\dot{\mathfrak{X}}(x)) \mathrm{d} s\right),
$$

provided that the Riemann integral on the right side exists in $\mathfrak{g}$. If this is the case for each $x \in I$, then $\alpha$ is of class $C^{1}$.

Proof. We let $\Phi: I \times[0,1] \ni(z, t) \mapsto \mathfrak{X}(z)$; and observe that $\alpha(z)=\int \Phi(z, \cdot)$ holds for each $z \in I$. Then, the first statement is clear from Theorem 3. For the second statement, we suppose that

$$
\dot{\alpha}(x)=\mathrm{d}_{e} \mathrm{~L}_{\exp (\mathfrak{X}(x))}\left(\int_{0}^{1} \operatorname{Ad}_{\exp (-s \cdot \mathfrak{X}(x))}(\dot{\mathfrak{X}}(x)) \mathrm{d} s\right) \quad \forall x \in I
$$

is well defined; i.e., that the Riemann integral on the right side exists for each $x \in I$. We fix $x \in I$ and $\delta>0$ with $[x-\delta, x+\delta] \subseteq I$; and observe that

$$
\mathbf{p}(\mathfrak{X}(x+h)-\mathfrak{X}(x)) \leq|h| \cdot \sup \{\cdot \mathfrak{p}(\dot{\mathfrak{X}}(z)) \mid z \in[x-\delta, x+\delta]\} \quad \forall \mathfrak{p} \in \mathfrak{P},|h| \leq \delta
$$

holds by (8). For each sequence $I \supseteq\left\{h_{n}\right\}_{n \in \mathbb{N}} \rightarrow 0$, we thus have

$$
\mathrm{D}_{\mathrm{c}} \supseteq\left\{\phi_{n}\right\}_{n \in \mathbb{N}} \rightarrow_{\mathfrak{m} . \mathrm{k}} \phi \in \mathrm{D}_{\mathrm{c}} \quad \text { for } \quad \phi:=\mathfrak{i}(\mathfrak{X}(x)) \quad \text { and } \quad \phi_{n}:=\mathfrak{i}\left(\mathfrak{X}\left(x+h_{n}\right)\right) \quad \forall n \in \mathbb{N} .
$$

Moreover, since $\mathfrak{X}$ is of class $C^{1}$, we have

$$
\mathrm{D}_{\mathrm{c}} \supseteq\left\{\psi_{n}\right\}_{n \in \mathbb{N}} \rightarrow_{\mathfrak{s} .0} \psi \in \mathrm{D}_{\mathrm{c}} \quad \text { for } \quad \psi:=\mathfrak{i}(\dot{\mathfrak{X}}(x)) \quad \text { and } \quad \psi_{n}:=\mathfrak{i}\left(\dot{\mathfrak{X}}\left(x+h_{n}\right)\right) \quad \forall n \in \mathbb{N} ;
$$

so that Lemma 18 shows

$$
\lim _{n \rightarrow \infty} \dot{\alpha}\left(x+h_{n}\right)=\lim _{(n, n) \rightarrow(\infty, \infty)} \xi\left(\phi_{n}, \psi_{n}\right)=\xi(\phi, \psi)=\dot{\alpha}(x) .
$$

This shows that $\dot{\alpha}$ is continuous at $x$. Since $x \in I$ was arbitrary, it follows that $\alpha$ is of class $C^{1}$.

For instance, we obtain the following generalization of Remark 2.3) in [7].

Example 1. Suppose that $G$ is Mackey c-continuous and abelian. Then, for each $\phi \in C^{0}\left(\left[r, r^{\prime}\right], \mathfrak{g}\right)$ with $\left[r, r^{\prime}\right] \ni t \mapsto \int_{r}^{t} \phi(s) \mathrm{d} s \in \operatorname{dom}[\exp ]$, we have, cf. Appendix A.5

$$
\int \phi=\exp \left(\int \phi(s) \mathrm{d} s\right) .
$$

In particular, if $\operatorname{dom}[\exp ]=\mathfrak{g}$ holds $\left(G\right.$ admits an exponential map), then $G$ is $C^{k}$-semiregular for $k \in \mathbb{N} \sqcup\{$ lip, $\infty\}$ if $\mathfrak{g}$ is $\mathrm{k}$-complete.

\subsection{Differentiation at Zero}

In this subsection, we prove Proposition 2, We start with some general remarks:

Let $\left[r, r^{\prime}\right] \in \mathfrak{K}$ and $\chi \in C^{0}\left(\left[r, r^{\prime}\right], \mathfrak{g}\right)$ be given. For $m \geq 1$ fixed, we define $t_{k}:=r+k / m \cdot\left(r^{\prime}-r\right)$ for $k=0, \ldots, m$; as well as $X_{k}:=\chi\left(t_{k}\right)$ for $k=0, \ldots, m-1$. We furthermore define $\chi_{m} \in C^{0}\left(\left[r, r^{\prime}\right], \mathfrak{g}\right)$ by $\chi_{m}(r):=X_{0}$ and

$$
\chi_{m}(t)=X_{k}+\left(t-t_{k}\right) /\left(t_{k+1}-t_{k}\right) \cdot\left(X_{k+1}-X_{k}\right) \quad \forall t \in\left(t_{k}, t_{k+1}\right], \quad k=0, \ldots, m-1 .
$$

Then, $\left\{\chi_{m}\right\}_{m \geq 1} \subseteq C^{0}\left(\left[r, r^{\prime}\right], \mathfrak{g}\right)$ constructed in this way, admits the following properties:

a) We have $\lim _{m} \cdot \mathfrak{p}_{\infty}\left(\chi-\chi_{m}\right)=0$ for each $\mathfrak{p} \in \mathfrak{P}$.

b) We have $\gamma_{h, m}:=h \cdot \mathrm{d}_{e} \Xi\left(\int_{r}^{\bullet} \chi_{m}(s) \mathrm{d} s\right) \in E$ for each $h \in \mathbb{R}$ and $m \geq 1$. 
c) Since $\operatorname{im}[\chi] \subseteq \mathfrak{g}$ is bounded, also $\left\{\operatorname{im}\left[\chi_{m}\right]\right\}_{m \geq 1} \subseteq \mathfrak{g},\left\{\operatorname{im}\left[\mathrm{d}_{e} \Xi\left(\int_{r}^{\bullet} \chi_{m}(s) \mathrm{d} s\right)\right]\right\}_{m \geq 1} \subseteq E$ are bounded. Thus,

- For each $\mathfrak{p} \in \mathfrak{P}$, there exists some $C_{\mathfrak{p}}>0$ with

$$
\mathfrak{p}_{\infty}\left(\gamma_{h, m}\right) \leq|h| \cdot C_{\mathfrak{p}} \quad \forall h \in \mathbb{R}, m \geq 1 .
$$

- For $\delta>0$ suitably small,

$$
\mu_{h, m}:=\Xi^{-1} \circ \gamma_{h, m} \in C^{1}\left(\left[r, r^{\prime}\right], G\right)
$$

is well defined for each $|h| \leq \delta, m \geq 1$; and we define

$$
\chi_{h, m}:=\delta^{r}\left(\mu_{h, m}\right) \stackrel{(14)}{=} h \cdot \omega\left(\gamma_{h, m}, \mathrm{~d}_{e} \Xi\left(\chi_{m}\right)\right) \quad \forall|h| \leq \delta, m \geq 1 .
$$

Moreover, for each fixed open neighbourhood $V \subseteq G$ of $e$, there exists some $0<\delta_{V} \leq \delta$ with

$$
V \ni \mu_{h, m}=\int_{r}^{\bullet} \chi_{h, m} \quad \forall|h| \leq \delta_{V}, m \geq 1 .
$$

Modifying the proof of Proposition 7 in [7, we obtain the

Proof of Proposition Q Suppose first that b) holds; and let

$$
A:=\Xi\left(\int_{r}^{\bullet} h_{n} \cdot \chi+h_{n} \cdot \varepsilon_{n}\right) \quad B:=\int_{r}^{\bullet}\left(\mathrm{d}_{e} \Xi \circ \chi\right)(s) \mathrm{d} s .
$$

Then, a) is clear from $\mathfrak{p}_{\infty}(A) \leq\left|h_{n}\right| \cdot \mathfrak{p}_{\infty}\left(1 / h_{n} \cdot A-B\right)+\left|h_{n}\right| \cdot \mathfrak{p}_{\infty}(B)$.

Suppose now that $a)$ holds - i.e. that we have $\lim _{n}^{\infty} \Xi\left(\int_{r}^{\bullet} \psi_{n}\right)=0$ with

$$
\psi_{n}:=h_{n} \cdot \chi+h_{n} \cdot \varepsilon_{n} \quad \forall n \in \mathbb{N} .
$$

We now have to show that for $\mathfrak{p} \in \mathfrak{P}$ fixed, the expression

$$
\Delta_{n}:=1 /\left|h_{n}\right| \cdot \overline{\mathfrak{p}}_{\infty}\left(\Xi\left(\int_{r}^{\bullet} \psi_{n}\right)-h_{n} \cdot \int_{r}^{\bullet} \mathrm{d}_{e} \Xi(\chi(s)) \mathrm{d} s\right)
$$

tends to zero for $n \rightarrow \infty$. For this, we choose $\mathfrak{p} \leq \mathfrak{q} \in \mathfrak{P}$ and $e \in V \subseteq G$ as in Lemma 9] and let

$$
\left\{\chi_{m}\right\}_{m \geq 1}, \quad\left\{\chi_{h, m}\right\}_{m \geq 1}, \quad\left\{\gamma_{h, m}\right\}_{m \geq 1}, \quad\left\{\mu_{h, m}\right\}_{m \geq 1}, \quad \delta_{V}>0
$$

be as above - with $\delta_{V}$ additionally such small that $\int_{r}^{\bullet} \psi_{n} \in V$ holds for each $n \in \mathbb{N}$ with $\left|h_{n}\right|<\delta_{V}$. We choose $\ell \in \mathbb{N}$ such large that $\left\{h_{n}\right\}_{n \geq \ell} \subseteq\left(-\delta_{V}, \delta_{V}\right)$ holds. Then, for each $n \geq \ell$ and $m \geq 1$, we obtain from (9) (second step), (59) and Lemma 9 (fifth step), as well as (58) (last step) that

$$
\begin{array}{rlrl}
\Delta_{n} & \leq 1 /\left|h_{n}\right| \cdot \mathfrak{p}_{\infty}\left(\Xi\left(\int_{r}^{\bullet} \psi_{n}\right)-h_{n} \cdot \mathrm{d}_{e} \Xi\left(\int_{r}^{\bullet} \chi_{m}(s) \mathrm{d} s\right)\right) & +\overline{\mathfrak{p}}_{\infty}\left(\int_{r}^{\bullet} \mathrm{d}_{e} \Xi(\chi(s)) \mathrm{d} s-\int_{r}^{\bullet} \mathrm{d}_{e} \Xi\left(\chi_{m}(s)\right) \mathrm{d} s\right) \\
& \leq 1 /\left|h_{n}\right| \cdot \mathfrak{p}_{\infty}\left(\Xi\left(\int_{r}^{\bullet} \psi_{n}\right)-\gamma_{h_{n}, m}\right) & & +\int\left(\mathfrak{p} \circ \mathrm{d}_{e} \Xi\right)\left(\chi(s)-\chi_{m}(s)\right) \mathrm{d} s \\
& =1 /\left|h_{n}\right| \cdot \mathfrak{p}_{\infty}\left(\Xi\left(\int_{r}^{\bullet} \psi_{n}\right)-\Xi\left(\mu_{h_{n}, m}\right)\right) & & +\int \mathfrak{p}\left(\chi(s)-\chi_{m}(s)\right) \mathrm{d} s \\
& =1 /\left|h_{n}\right| \cdot \mathfrak{p}_{\infty}\left(\Xi\left(\int_{r}^{\bullet} \psi_{n}\right)-\Xi\left(\int_{r}^{\bullet} \chi_{h_{n}, m}\right)\right) & & +\int \mathfrak{p}\left(\chi(s)-\chi_{m}(s)\right) \mathrm{d} s \\
& \leq 1 /\left|h_{n}\right| \cdot \int \cdot \mathfrak{q}\left(\psi_{n}(s)-\chi_{h_{n}, m}(s)\right) \mathrm{d} s & & +\int \mathfrak{p}\left(\chi(s)-\chi_{m}(s)\right) \mathrm{d} s \\
& \leq \int \mathfrak{q}\left(\varepsilon_{n}(s)\right) \mathrm{d} s+\int \cdot \mathfrak{q}\left(\chi(s)-\omega\left(\gamma_{h_{n}, m}(s), \mathrm{d}_{e} \Xi\left(\chi_{m}(s)\right)\right)\right) \mathrm{d} s+\left(r^{\prime}-r\right) \cdot \mathfrak{p}_{\infty}\left(\chi-\chi_{m}\right)
\end{array}
$$

holds. By Lebesgue's dominated convergence theorem and $i$ ), ii), the first term tends to zero for $n \rightarrow \infty$; and, by $\mathbf{a}$ ), the third term tends to zero for $m \rightarrow \infty$. Thus, $\varepsilon>0$ given, there exists some $\ell_{\varepsilon} \geq \ell$, such that both the first-, and the third term is bounded by $\varepsilon / 4$ for all $m, n \geq \ell_{\varepsilon}$. Moreover, since $\chi=\omega\left(0, \mathrm{~d}_{e} \Xi(\chi)\right)$ holds (second step), we can estimate the second term by

$$
\begin{aligned}
\int \cdot \mathfrak{q}(\chi(s)- & \left.\omega\left(\gamma_{h_{n}, m}(s), \mathrm{d}_{e} \Xi\left(\chi_{m}(s)\right)\right)\right) \mathrm{d} s \\
\leq & \left(r^{\prime}-r\right) \cdot \mathfrak{q}_{\infty}\left(\chi-\omega\left(\gamma_{h_{n}, m}, \mathrm{~d}_{e} \Xi\left(\chi_{m}\right)\right)\right) \\
= & \left(r^{\prime}-r\right) \cdot \mathfrak{q}_{\infty}\left(\omega\left(0, \mathrm{~d}_{e} \Xi(\chi)\right)-\omega\left(\gamma_{h_{n}, m}, \mathrm{~d}_{e} \Xi\left(\chi_{m}\right)\right)\right) \\
\leq & \left(r^{\prime}-r\right) \cdot \mathfrak{q}_{\infty}\left(\omega\left(0, \mathrm{~d}_{e} \Xi(\chi)\right)-\omega\left(\gamma_{h_{n}, m}, \mathrm{~d}_{e} \Xi(\chi)\right)\right) \\
& +\left(r^{\prime}-r\right) \cdot \mathfrak{q}_{\infty}\left(\omega\left(\gamma_{h_{n}, m}, \mathrm{~d}_{e} \Xi\left(\chi-\chi_{m}\right)\right)\right) .
\end{aligned}
$$


- Since $\operatorname{im}[\chi]$ is compact, increasing $\ell_{\varepsilon}$ if necessary, by (57), we can achieve that the fourth line in (60) is bounded by $\varepsilon / 4$ for each $n, m \geq \ell_{\varepsilon}$.

- To estimate the last line in (60), we choose $\mathfrak{q} \leq \mathfrak{m} \in \mathfrak{P}$ as in (15); and increase $\ell_{\varepsilon}$ in such a way (use (57)) that $\mathfrak{m}_{\infty}\left(\gamma_{h_{n}, m}\right) \leq 1$ holds for all $n, m \geq \ell_{\varepsilon}$; thus,

$$
\mathfrak{q}_{\infty}\left(\omega\left(\gamma_{h_{n}, m}, \mathrm{~d}_{e} \Xi\left(\chi-\chi_{m}\right)\right)\right) \stackrel{(15)}{\leq} \mathfrak{m}_{\infty}\left(\chi-\chi_{m}\right) .
$$

Then, it is clear from a) that for $\ell_{\varepsilon}^{\prime} \geq \ell_{\varepsilon}$ suitably large, the last line in (60) is bounded by $\varepsilon / 4$ for all $m, n \geq \ell_{\varepsilon}^{\prime}$.

We thus have $\Delta_{n} \leq \varepsilon$ for each $n \geq \ell_{\varepsilon}^{\prime} \in \mathbb{N}$; which shows $\lim _{n} \Delta_{n}=0$.

\section{Extension: The Metrizable Category}

We recall that a Hausdorff locally convex vector space is said to be metrizable if it admits a metric that generates the topology thereon. We furthermore recall that $G$ is said to be $C^{k}$-regular if $G$ is $C^{k}$-semiregular such that evol $\mathrm{k}_{\mathrm{k}}$ is smooth w.r.t. the $C^{k}$-topology.

After this paper had been put on the arXiv, the author's attention was drawn by Glöckner and Schmeding to the fact that in metrizable locally convex vector spaces, convergence of a sequence implies its Mackey convergence (and vice versa). Specifically, it was argued that the following two results will hold:

Lemma 19. Suppose that $\mathfrak{g}$ is metrizable; and let $k \in \mathbb{N} \sqcup\{\operatorname{lip}, \infty, \mathrm{c}\}$. Then, the following conditions are equivalent:

i) $G$ is $C^{k}$-continuous.

ii) $G$ is sequentially k-continuous.

iii) $G$ is Mackey k-continuous.

Corollary 7. Suppose that $\mathfrak{g}$ is a Fréchet space; and let $k \in \mathbb{N} \sqcup\{\infty\}$. Then, $G$ is $C^{k}$-regular if and only if $G$ is $C^{k}$-semiregular.

Proof. The one implication is evident. Suppose thus that $G$ is $C^{k}$-semiregular. Then, $G$ is Mackey k-continuous by Theorem 1, so that evol $_{\mathrm{k}}$ is $C^{k}$-continuous by Lemma 19. Since $\mathfrak{g}$ is complete (thus, integral complete and Mackey complete), Theorem 4 in [7] shows that evol $_{\mathrm{k}}$ is smooth, i.e., that $G$ is $C^{k}$-regular.

The rest of this section is dedicated to a selfcontained proof of Lemma 19,

\section{Some Standard Facts:}

Let $F$ be a Hausdorff locally convex vector space, with system of continuous seminorms $\mathfrak{Q}$. A subsystem $\mathfrak{H} \subseteq \mathfrak{Q}$ is said to be a fundamental system if $\left\{\mathrm{B}_{\mathfrak{h}, \varepsilon}(0)\right\}_{\mathfrak{h} \in \mathfrak{H}, \varepsilon>0}$ is a local base of zero in $F$. We recall that

Lemma 20. Let $\mathfrak{H} \subseteq \mathfrak{Q}$ be a fundamental system, and $\mathfrak{S} \subseteq \mathfrak{Q}$ a subsystem. Then, the following statements are equivalent:

1) $\mathfrak{S}$ is a fundamental system.

2) To each $\mathfrak{h} \in \mathfrak{H}$, there exist $c>0$ and $\mathfrak{s} \in \mathfrak{S}$ with $\mathfrak{h} \leq c \cdot \mathfrak{s}$.

Proof. If $\mathfrak{S}$ is a fundamental system, then 2) follows from Proposition 22.6 in [14] when applied to the identity $\operatorname{id}_{F}$. Suppose thus that 2) holds; and let $V \subseteq F$ be open with $0 \in V$. We choose $\mathfrak{h} \in \mathfrak{H}$ with $\mathrm{B}_{\mathfrak{h}, \varepsilon}(0) \subseteq V$, fix $c>0$ and $\mathfrak{s} \in \mathfrak{S}$ with $\mathfrak{h} \leq c \cdot \mathfrak{s} ;$ and observe that $\mathrm{B}_{\mathfrak{s}, \frac{\varepsilon}{c}}(0) \subseteq \mathrm{B}_{\mathfrak{h}, \varepsilon}(0) \subseteq V$ holds. Since $\mathrm{B}_{\mathfrak{s}, \frac{\varepsilon}{c}}(0) \subseteq F$ is open, 1 ) follows. 
Lemma 21. The following statements are equivalent:

1) $F$ is metrizable.

2) There exists a countable fundamental system $\{\mathfrak{q}[m] \mid m \in \mathbb{N}\} \subseteq \mathfrak{Q}$.

3) There exists $\{\mathfrak{q}[m] \mid m \in \mathbb{N}\} \subseteq \mathfrak{Q}$ as in $[\mathbf{2})$ with $\mathfrak{q}[m] \leq \mathfrak{q}[m+1]$ for each $m \in \mathbb{N}$.

Proof. The equivalence of 1) and 2) is covered by Proposition 25.1 in [14. It is furthermore clear that 3) implies 2). Let thus $\{\mathfrak{q}[m] \mid m \in \mathbb{N}\} \subseteq \mathfrak{Q}$ be as in 2), and define

$$
\mathfrak{S}:=\{\mathfrak{o}[m] \equiv \mathfrak{q}[0]+\ldots+\mathfrak{q}[m] \mid m \in \mathbb{N}\} \subseteq \mathfrak{Q} .
$$

Since $\mathfrak{q}[m] \leq \mathfrak{o}[m]$ holds for each $m \in \mathbb{N}$, Lemma 20 shows that $\mathfrak{S}$ is a fundamental system; which establishes 3).

Let $\mathfrak{H} \subseteq \mathfrak{Q}$ be a fundamental system. We write $\left\{X_{n}\right\}_{n \in \mathbb{N}} \rightarrow_{\mathfrak{m}} X$ for $\left\{X_{n}\right\}_{n \in \mathbb{N}} \subseteq F$ and $X \in F$ if

$$
\mathfrak{h}\left(X-X_{n}\right) \leq \mathfrak{c}_{\mathfrak{h}} \cdot \lambda_{n} \quad \forall n \geq \mathfrak{l}_{\mathfrak{h}}, \mathfrak{h} \in \mathfrak{H}
$$

holds for certain $\left\{\mathfrak{c}_{\mathfrak{h}}\right\}_{\mathfrak{h} \in \mathfrak{H}} \subseteq \mathbb{R}_{\geq 0},\left\{\mathfrak{l}_{\mathfrak{h}}\right\}_{\mathfrak{h} \in \mathfrak{H}} \subseteq \mathbb{N}$, and $\mathbb{R}_{\geq 0} \supseteq\left\{\lambda_{n}\right\}_{n \in \mathbb{N}} \rightarrow 0$.

Remark 10. It is immediate from Lemma 20 that the definition made in (61) does not depend on the explicit choice of the fundamental system $\mathfrak{H}$.

We obtain

Lemma 22. Suppose that $F$ is metrizable; and let $\left\{X_{n}\right\}_{n \in \mathbb{N}} \subseteq F$ be a sequence with $\left\{X_{n}\right\}_{n \in \mathbb{N}} \rightarrow$ $X \in F$. Then, we have $\left\{X_{n}\right\}_{n \in \mathbb{N}} \rightarrow_{\mathfrak{m}} X$.

Proof. Although this statement is well known from the literature (cf., e.g., 4. Proposition in Sect. 10.1 in [10]), for completeness reasons, we provide an elementary proof that is adapted to our particular formulation of Mackey convergence, cf. Appendix A.6.

We recall that the $C^{k}$-topology on $\mathrm{F}_{k}:=C^{k}([0,1], F)$ for $k \in \mathbb{N} \sqcup\{$ lip, $\infty, \mathrm{c}\}$ is the Hausdorff locally convex topology that is generated by the seminorms $\mathfrak{H}_{k}:=\left\{\mathfrak{q}_{\infty}^{\mathrm{s}} \mid \mathfrak{q} \in \mathfrak{Q}, \mathrm{s} \preceq k\right\}$ (cf. Sect. 2.1.1). Since $\mathfrak{H}_{k}$ is a fundamental system, the definition made in (61) coincides with the definition made in (21). We furthermore recall that

Lemma 23. If $F$ is metrizable, then $C^{k}([0,1], F)$ is metrizable for each $k \in \mathbb{N} \sqcup\{$ lip, $\infty, c\}$.

Proof. Confer, e.g., Appendix A.7.

\section{The Proof of Lemma 19:}

We obtain from Lemma 21 and Lemma 23,

Corollary 8. Suppose that $\mathfrak{g}$ is metrizable; and let $k \in \mathbb{N} \sqcup\{$ lip, $\infty, c\}$. Then, $G$ is sequentially $\mathrm{k}$-continuous if and only if $G$ is Mackey k-continuous.

Proof. Let $\left\{\phi_{n}\right\}_{n \in \mathbb{N}} \subseteq \mathrm{D}_{\mathrm{k}}$, and $\phi \in \mathrm{D}_{\mathrm{k}}$ be given.

- Evidently, $\left\{\phi_{n}\right\}_{n \in \mathbb{N}} \rightarrow_{\text {m.k }} \phi$ implies $\left\{\phi_{n}\right\}_{n \in \mathbb{N}} \rightarrow_{\mathfrak{s . k}} \phi$; so that $G$ is Mackey k-continuous if $G$ is sequentially k-continuous.

- By Lemma 23, $C^{k}([0,1], \mathfrak{g})$ is metrizable. Lemma 22 thus shows that $\left\{\phi_{n}\right\}_{n \in \mathbb{N}} \rightarrow_{\mathfrak{s . k}} \phi$ implies $\left\{\phi_{n}\right\}_{n \in \mathbb{N}} \rightarrow_{\mathfrak{m} . k} \phi$. Consequently, $G$ is sequentially k-continuous if $G$ is Mackey k-continuous.

We are ready for the

Proof of Lemma [19. The equivalence of ii) and iii) is covered by Corollary 8. Moreover, since Lemma 23 shows that $C^{k}([0,1], \mathfrak{g})$ is metrizable (thus, first countable), the equivalence of i) and ii) is clear from Remark 75 ) as well as Remark 76 ). 


\section{APPENDIX}

\section{A Appendix}

\section{A.1 Bastiani's Differential Calculus}

In this Appendix, we recall the differential calculus from [2, 6, 15, 18, cf. also Sect. 3.3.1 in [7].

Let $E$ and $F$ be Hausdorff locally convex vector spaces. A map $f: U \rightarrow E$, with $U \subseteq F$ open, is said to be differentiable at $x \in U$ if

$$
\left(D_{v} f\right)(x):=\lim _{h \rightarrow 0} 1 / h \cdot(f(x+h \cdot v)-f(x)) \in E
$$

exists for each $v \in F$. Then, $f$ is said to be differentiable if it is differentiable at each $x \in U$. More generally, $f$ is said to be $k$-times differentiable for $k \geq 1$ if

$$
D_{v_{k}, \ldots, v_{1}} f \equiv D_{v_{k}}\left(D_{v_{k-1}}\left(\ldots\left(D_{v_{1}}(f)\right) \ldots\right)\right): U \rightarrow E
$$

is well defined for each $v_{1}, \ldots, v_{k} \in F$ - implicitly meaning that $f$ is $p$-times differentiable for each $1 \leq p \leq k$. In this case, we define

$$
\mathrm{d}_{x}^{p} f\left(v_{1}, \ldots, v_{p}\right) \equiv \mathrm{d}^{p} f\left(x, v_{1}, \ldots, v_{p}\right):=D_{v_{p}, \ldots, v_{1}} f(x) \quad \forall x \in U, v_{1}, \ldots, v_{p} \in F
$$

for $p=1, \ldots, k$; and let $\mathrm{d} f \equiv \mathrm{d}^{1} f$, as well as $\mathrm{d}_{x} f \equiv \mathrm{d}_{x}^{1} f$ for each $x \in U$. Then, $f$ is said to be

- of class $C^{0}$ if it is continuous - In this case, we let $\mathrm{d}^{0} f \equiv f$.

- of class $C^{k}$ for $k \geq 1$ if it is $k$-times differentiable, such that

$$
\mathrm{d}^{p} f: U \times F^{p} \rightarrow E, \quad\left(x, v_{1}, \ldots, v_{p}\right) \mapsto D_{v_{p}, \ldots, v_{1}} f(x)
$$

is continuous for each $p=0, \ldots, k$. In this case, $\mathrm{d}_{x}^{p} f$ is symmetric and $p$-multilinear for each $x \in U$ and $p=1, \ldots, k$, cf. [2].

- of class $C^{\infty}$ if it is of class $C^{k}$ for each $k \in \mathbb{N}$.

We have the following differentiation rules [2]:

a) A map $f: F \supseteq U \rightarrow E$ is of class $C^{k}$ for $k \geq 1$ if $\mathrm{d} f$ is of class $C^{k-1}$ when considered as a map $F^{\prime} \supseteq U^{\prime} \rightarrow E$ for $F^{\prime} \equiv F \times F$ and $U^{\prime} \equiv U \times F$.

b) If $f: U \rightarrow F$ is linear and continuous, then $f$ is smooth; with $\mathrm{d}_{x}^{1} f=f$ for each $x \in E$, as well as $\mathrm{d}^{k} f=0$ for each $k \geq 2$.

c) Suppose that $f: F \supseteq U \rightarrow U^{\prime} \subseteq F^{\prime}$ and $f^{\prime}: F^{\prime} \supseteq U^{\prime} \rightarrow F^{\prime \prime}$ are of class $C^{k}$ for $k \geq 1$, for Hausdorff locally convex vector spaces $F, F^{\prime}, F^{\prime \prime}$. Then, $f^{\prime} \circ f: U \rightarrow F^{\prime \prime}$ is of class $C^{k}$ with

$$
\mathrm{d}_{x}\left(f^{\prime} \circ f\right)=\mathrm{d}_{f(x)} f^{\prime} \circ \mathrm{d}_{x} f \quad \forall x \in U .
$$

d) Let $F_{1}, \ldots, F_{m}, E$ be Hausdorff locally convex vector spaces, and let $f: F_{1} \times \ldots \times F_{m} \supseteq U \rightarrow E$ be of class $C^{0}$. Then, $f$ is of class $C^{1}$ if and only if for $p=1, \ldots, m$, the "partial derivative"

$\partial_{p} f: U \times F_{p} \ni\left(\left(x_{1}, \ldots, x_{m}\right), v_{p}\right) \mapsto \lim _{h \rightarrow 0} 1 / h \cdot\left(f\left(x_{1}, \ldots, x_{p}+h \cdot v_{p}, \ldots, x_{m}\right)-f\left(x_{1}, \ldots, x_{m}\right)\right)$

exists in $E$, and is continuous. In this case, we have

$$
\begin{aligned}
\mathrm{d}_{\left(x_{1}, \ldots, x_{m}\right)} f\left(v_{1}, \ldots, v_{m}\right) & =\sum_{p=1}^{m} \partial_{p} f\left(\left(x_{1}, \ldots, x_{m}\right), v_{p}\right) \\
& =\sum_{p=1}^{m} \mathrm{~d} f\left(\left(x_{1}, \ldots, x_{m}\right),\left(0, \ldots, 0, v_{p}, 0, \ldots, 0\right)\right)
\end{aligned}
$$

for each $\left(x_{1}, \ldots, x_{m}\right) \in U$, and $v_{p} \in F_{p}$ for $p=1, \ldots, m$. 


\section{A.2 Proof of Lemma 7}

In this appendix, we prove

Lemma [7. Let $\left[r, r^{\prime}\right] \in \mathfrak{K}$, and $\phi \in \mathfrak{D}_{\left[r, r^{\prime}\right]}$ be fixed. Then, for each $\mathfrak{p} \in \mathfrak{P}$, there exists some $\mathfrak{p} \leq \mathfrak{q} \in \mathfrak{P}$ with

$$
\mathfrak{p}_{\infty}^{\operatorname{lip}}\left(\operatorname{Ad}_{\left[\int_{r}^{\bullet} \phi\right]^{-1}}(\psi)\right) \leq \cdot \mathfrak{q}_{\infty}^{\operatorname{lip}}(\psi) \quad \forall \psi \in C^{\text {lip }}\left(\left[r, r^{\prime}\right], \mathfrak{g}\right)
$$

Proof. By definition, there exists some $\mu \in C^{1}(I, G)$, for $I$ an open interval containing $\left[r, r^{\prime}\right]$, with $\left.\delta^{r}(\mu)\right|_{\left[r, r^{\prime}\right]}=\phi$ and $\mu(r)=e$. We now have to show that

$$
C^{\operatorname{lip}}\left(\left[r, r^{\prime}\right], \mathfrak{g}\right) \ni \chi:\left[r, r^{\prime}\right] \ni t \mapsto \operatorname{Ad}_{\mu^{-1}(t)}(\psi(t))
$$

holds, for each fixed $\psi \in C^{\text {lip }}\left(\left[r, r^{\prime}\right], \mathfrak{g}\right)$. For this, we let $\mathfrak{p} \in \mathfrak{P}$ be fixed; and obtain

$$
\mathfrak{. p}\left(\chi(t)-\chi\left(t^{\prime}\right)\right) \leq \mathfrak{p}\left(\operatorname{Ad}_{\mu^{-1}(t)}\left(\psi(t)-\psi\left(t^{\prime}\right)\right)\right)+\cdot \mathfrak{p}\left(\left(\operatorname{Ad}_{\mu^{-1}(t)}-\operatorname{Ad}_{\mu^{-1}\left(t^{\prime}\right)}\right)\left(\psi\left(t^{\prime}\right)\right)\right) .
$$

- We let $\mathrm{C}:=\operatorname{im}\left[\mu^{-1}\right]$, choose $\mathfrak{p} \leq \mathfrak{w} \in \mathfrak{P}$ as in $\left.\mathrm{I}\right)$ for $\mathfrak{v} \equiv \mathfrak{p}$ there; and obtain

$$
\begin{aligned}
\mathfrak{p}(\chi(t)) & \leq \mathfrak{w}(\psi(t)) & & \forall t \in\left[r, r^{\prime}\right], \\
\mathfrak{\cdot p}\left(\operatorname{Ad}_{\mu^{-1}(t)}\left(\psi(t)-\psi\left(t^{\prime}\right)\right)\right) & \leq \mathfrak{w}\left(\psi(t)-\psi\left(t^{\prime}\right)\right) \leq \operatorname{Lip}(\cdot \mathfrak{w}, \psi) \cdot\left|t-t^{\prime}\right| & & \forall t, t^{\prime} \in\left[r, r^{\prime}\right] .
\end{aligned}
$$

- The map $\alpha: I \times \mathfrak{g} \ni(s, X) \rightarrow \partial_{s} \operatorname{Ad}_{\mu^{-1}(s)}(X)$ is well defined, continuous, and linear in the second argument. By Lemma 2 applied to $\mathrm{K} \equiv \mathrm{C}$, there thus exists some $\mathfrak{p} \leq \mathfrak{m} \in \mathfrak{P}$ with

$$
(\cdot \mathfrak{p} \circ \alpha)(s, X) \leq \mathfrak{m}(X) \quad \forall s \in\left[r, r^{\prime}\right], X \in \mathfrak{g} .
$$

Then, we obtain from (8) that

$$
\begin{aligned}
\cdot \mathfrak{p}\left(\left(\operatorname{Ad}_{\mu^{-1}(t)}-\operatorname{Ad}_{\mu^{-1}\left(t^{\prime}\right)}\right)\left(\psi\left(t^{\prime}\right)\right)\right) & \leq \int_{t^{\prime}}^{t} \cdot \mathfrak{p}\left(\partial_{s} \operatorname{Ad}_{\mu^{-1}(s)}\left(\psi\left(t^{\prime}\right)\right)\right) \mathrm{d} s \\
& =\int_{t^{\prime}}^{t}(\mathfrak{p} \circ \alpha)\left(s, \psi\left(t^{\prime}\right)\right) \mathrm{d} s \\
& \leq \mathfrak{m}_{\infty}(\psi) \cdot\left|t-t^{\prime}\right|
\end{aligned}
$$

holds, for each $t, t^{\prime} \in\left[r, r^{\prime}\right]$ with $t^{\prime} \leq t$.

We choose $\mathfrak{q} \in \mathfrak{P}$ with $\mathfrak{q} \geq 2 \cdot \max (\mathfrak{m}, \mathfrak{w})$ (i.e., $\mathfrak{p}, \mathfrak{m}, \mathfrak{w} \leq \mathfrak{q}$ ); and obtain

$$
\cdot \mathfrak{p}_{\infty}(\chi) \stackrel{\text { 63) }}{\leq} \cdot \mathfrak{w}_{\infty}(\psi) \leq \cdot \mathfrak{q}_{\infty}(\psi) .
$$

We furthermore obtain from (62), (64), (65) that

$$
\mathfrak{\cdot p}\left(\chi(t)-\chi\left(t^{\prime}\right)\right) \leq \operatorname{Lip}(\cdot \mathfrak{w}, \psi) \cdot\left|t-t^{\prime}\right|+\mathfrak{m}_{\infty}(\psi) \cdot\left|t-t^{\prime}\right| \leq \mathfrak{q}_{\infty}^{\operatorname{lip}}(\psi) \cdot\left|t-t^{\prime}\right|
$$

holds for each $t, t^{\prime} \in\left[r, r^{\prime}\right]$; thus,

$$
\operatorname{Lip}(\cdot \mathfrak{p}, \chi) \leq \cdot \mathfrak{q}_{\infty}^{\operatorname{lip}}(\psi) \quad \stackrel{(66)}{\Longrightarrow} \quad \mathfrak{p p}_{\infty}^{\operatorname{lip}}(\chi) \leq \cdot \mathfrak{q}_{\infty}^{\operatorname{lip}}(\psi)
$$

which proves the claim. 


\section{A.3 Proof of Equation (38)}

In this appendix, we show

$$
\operatorname{Lip}\left(\cdot \mathfrak{p},\left.\phi\right|_{\left[t_{n+1}, 1\right]}\right) \leq 2 \cdot C[\boldsymbol{\rho}, 1]^{2} \cdot \max \left(\delta_{0}^{-8} \cdot \mathfrak{p}_{\infty}^{\operatorname{lip}}\left(\phi_{0}\right), \ldots, \delta_{n}^{-8} \cdot \mathfrak{\cdot p} \mathfrak{p}_{\infty}^{\operatorname{lip}}\left(\phi_{n}\right)\right) .
$$

Proof of Equation (38). We let $\varphi_{n}:=\rho_{n} \cdot\left(\phi_{n} \circ \varrho_{n}\right)$ for each $n \in \mathbb{N}$; so that

$$
\operatorname{Lip}\left(\cdot \mathfrak{p}, \boldsymbol{\varphi}_{n}\right) \leq 2 \cdot \delta_{n}^{-8} \cdot C[\boldsymbol{\rho}, 1]^{2} \cdot \mathfrak{p}_{\infty}^{\operatorname{lip}}\left(\phi_{n}\right)
$$

holds by (35). Then, for $t, t^{\prime} \in\left[t_{\ell+1}, t_{\ell}\right]$ with $\ell \in \mathbb{N}$, we have

$$
\begin{aligned}
\cdot \mathfrak{p}\left(\phi(t)-\phi\left(t^{\prime}\right)\right) & =\mathfrak{\cdot p}\left(\boldsymbol{\varphi}_{\ell}(t)-\boldsymbol{\varphi}_{\ell}\left(t^{\prime}\right)\right) \\
& \leq \operatorname{Lip}\left(\cdot \mathfrak{p}, \boldsymbol{\varphi}_{\ell}\right) \cdot\left|t-t^{\prime}\right| \\
& \leq 2 \cdot C[\boldsymbol{\rho}, 1]^{2} \cdot \delta_{\ell}^{-8} \cdot \mathfrak{p}_{\infty}^{\operatorname{lip}}\left(\phi_{\ell}\right) \cdot\left|t-t^{\prime}\right| .
\end{aligned}
$$

Moreover, for $t \in\left[t_{(\ell+1)+m}, t_{\ell+m}\right]$ and $t^{\prime} \in\left[t_{\ell+1}, t_{\ell}\right]$, with $m \geq 1$ and $\ell \in \mathbb{N}$, we have

$$
\begin{aligned}
\mathfrak{p}\left(\phi(t)-\phi\left(t^{\prime}\right)\right) \leq & \mathfrak{p}\left(\phi(t)-\phi\left(t_{\ell+m}\right)\right) \\
& +\sum_{k=m-1}^{1} \cdot \mathfrak{p}\left(\phi\left(t_{(\ell+1)+k}\right)-\phi\left(t_{\ell+k}\right)\right) \\
& +\mathfrak{p}\left(\phi\left(t_{\ell+1}\right)-\phi\left(t^{\prime}\right)\right) \\
\leq & \mathfrak{p}\left(\boldsymbol{\varphi}_{\ell+m}(t)-\boldsymbol{\varphi}_{\ell+m}\left(t_{\ell+m}\right)\right) \\
& +\sum_{k=m-1}^{1} \cdot \mathfrak{p}\left(\boldsymbol{\varphi}_{\ell+k}\left(t_{(\ell+1)+k}\right)-\boldsymbol{\varphi}_{\ell+k}\left(t_{\ell+k}\right)\right) \\
& +\cdot \mathfrak{p}\left(\boldsymbol{\varphi}_{\ell}\left(t_{\ell+1}\right)-\boldsymbol{\varphi}_{\ell}\left(t^{\prime}\right)\right) \\
\leq & \operatorname{Lip}\left(\cdot \mathfrak{p}, \boldsymbol{\varphi}_{\ell+m}\right) \cdot\left|t-t_{\ell+m}\right| \\
& +\sum_{k=m-1}^{1} \operatorname{Lip}\left(\mathfrak{p}, \boldsymbol{\varphi}_{\ell+k}\right) \cdot\left|t_{(\ell+1)+k}-t_{\ell+k}\right| \\
& +\operatorname{Lip}\left(\cdot \mathfrak{p}, \boldsymbol{\varphi}_{\ell}\right) \cdot\left|t_{\ell+1}-t^{\prime}\right| \\
\leq & 2 \cdot \delta_{\ell+m}^{-8} \cdot C[\boldsymbol{\rho}, 1]^{2} \cdot \mathfrak{p} \mathfrak{p}_{\infty}^{\operatorname{lip}}\left(\phi_{\ell+m}\right) \cdot\left|t-t_{\ell+m}\right| \\
& +\sum_{k=m-1}^{1} 2 \cdot \delta_{\ell+k}^{-8} \cdot C[\boldsymbol{\rho}, 1]^{2} \cdot \mathfrak{p}_{\infty}^{\operatorname{lip}}\left(\phi_{\ell+k}\right) \cdot\left|t_{(\ell+1)+k}-t_{\ell+k}\right| \\
& +2 \cdot \delta_{\ell}^{-8} \cdot C[\boldsymbol{\rho}, 1]^{2} \cdot \mathfrak{p}_{\infty}^{\operatorname{lip}}\left(\phi_{\ell}\right) \cdot\left|t_{\ell+1}-t^{\prime}\right| \\
\leq & 2 \cdot C[\boldsymbol{\rho}, 1]^{2} \cdot \max _{0 \leq k \leq m}\left(\delta_{\ell+k}^{-8} \cdot \mathfrak{p}_{\infty}^{\operatorname{lip}}\left(\phi_{\ell+k}\right)\right) \cdot\left|t-t^{\prime}\right| .
\end{aligned}
$$

Combining (67) with (68), we obtain (38).

\section{A.4 Proof of Lemma 18}

In this appendix, we prove

Lemma 18. Suppose that $G$ is Mackey k-continuous for $k \in \mathbb{N} \sqcup\{\operatorname{lip}, \infty, \mathrm{c}\}$. Suppose furthermore that we are given $\mathfrak{D}_{\left[r, r^{\prime}\right]}^{k} \supseteq\left\{\phi_{n}\right\}_{n \in \mathbb{N}} \rightarrow_{\text {m.k }} \phi \in \mathfrak{D}_{\left[r, r^{\prime}\right]}^{k}$ as well as $\mathfrak{D}_{\left[r, r^{\prime}\right]}^{k} \supseteq\left\{\psi_{\alpha}\right\}_{\alpha \in I} \rightarrow_{\mathfrak{n} .0} \psi \in \mathfrak{D}_{\left[r, r^{\prime}\right]}^{k}$ for $\left[r, r^{\prime}\right] \in \mathfrak{K}$, such that the expressions

$$
\begin{aligned}
\xi(\phi, \psi) & :=\mathrm{d}_{e} \mathrm{~L}_{\boldsymbol{}}\left(\int \operatorname{Ad}_{\left[\int_{r}^{s} \phi\right]^{-1}}(\psi(s)) \mathrm{d} s\right) \\
\xi\left(\phi_{n}, \psi_{\alpha}\right) & :=\mathrm{d}_{e} \mathrm{~L}_{\int \phi_{n}}\left(\int \operatorname{Ad}_{\left[\int_{r}^{s} \phi_{n}\right]^{-1}}\left(\psi_{\alpha}(s)\right) \mathrm{d} s\right) \quad \forall n \in \mathbb{N}
\end{aligned}
$$

are well defined; i.e., such that the occurring Riemann integrals exist in $\mathfrak{g}$. Then, we have

$$
\lim _{(n, \alpha)} \xi\left(\phi_{n}, \psi_{\alpha}\right)=\xi(\phi, \psi) .
$$


For this, we first show the following analogue to Lemma 41 in [7].

Lemma 24. Suppose that $G$ is Mackey k-continuous for $k \in \mathbb{N} \sqcup\{\operatorname{lip}, \infty, \mathrm{c}\}$; and let $\left[r, r^{\prime}\right] \in \mathfrak{K}$ be fixed. Let $\Gamma: G \times \mathfrak{g} \rightarrow \mathfrak{g}$ be continuous; and define

$$
\widehat{\Gamma}: \mathfrak{D}_{\left[r, r^{\prime}\right]}^{k} \times C^{k}\left(\left[r, r^{\prime}\right], \mathfrak{g}\right) \rightarrow \overline{\mathfrak{g}}, \quad(\phi, \psi) \mapsto \int \Gamma\left(\int_{r}^{s} \phi, \psi(s)\right) \mathrm{d} s .
$$

Then, for each sequence $\mathfrak{D}_{\left[r, r^{\prime}\right]}^{k} \supseteq\left\{\phi_{n}\right\}_{n \in \mathbb{N}} \rightarrow_{\mathfrak{m} . \mathrm{k}} \phi \in \mathfrak{D}_{\left[r, r^{\prime}\right]}^{k}$ and each net $C^{k}\left(\left[r, r^{\prime}\right], \mathfrak{g}\right) \supseteq\left\{\psi_{\alpha}\right\}_{\alpha \in I} \rightarrow_{\mathfrak{n} .0}$ $\psi \in C^{k}\left(\left[r, r^{\prime}\right], \mathfrak{g}\right)$, we have

$$
\lim _{(n, \alpha)} \widehat{\Gamma}\left(\phi_{n}, \psi_{\alpha}\right)=\widehat{\Gamma}(\phi, \psi) .
$$

Proof. By (9), it suffices to show that for

$$
\widetilde{\Gamma}: \mathfrak{D}_{\left[r, r^{\prime}\right]}^{k} \times C^{k}\left(\left[r, r^{\prime}\right], \mathfrak{g}\right) \rightarrow C^{0}\left(\left[r, r^{\prime}\right], \mathfrak{g}\right), \quad(\phi, \psi) \mapsto\left[t \mapsto \Gamma\left(\int_{r}^{t} \phi, \psi(t)\right)\right],
$$

we have $\lim _{(n, \alpha)} \widetilde{\Gamma}\left(\phi_{n}, \psi_{\alpha}\right)=\widetilde{\Gamma}(\phi, \psi)$ w.r.t. the $C^{0}$-topology; i.e., that for $\mathfrak{p} \in \mathfrak{P}$ and $\varepsilon>0$ fixed, there exist $N_{\varepsilon} \in \mathbb{N}$ and $\alpha_{\varepsilon} \in I$ with

$$
\mathfrak{p}_{\infty}\left(\widetilde{\Gamma}\left(\phi_{n}, \psi_{\alpha}\right)-\widetilde{\Gamma}(\phi, \psi)\right)<\varepsilon \quad \forall n \geq N_{\varepsilon}, \alpha \geq \alpha_{\varepsilon} .
$$

For this, we let $\mu:=\int_{r}^{\bullet} \phi$, and consider the continuous map

$$
\alpha: G \times \mathfrak{g} \times G \times \mathfrak{g} \rightarrow \mathfrak{g}, \quad\left((g, X),\left(g^{\prime}, X^{\prime}\right)\right) \mapsto \mathfrak{p}\left(\Gamma(g, X)-\Gamma\left(g^{\prime}, X^{\prime}\right)\right) .
$$

Then, for $t \in\left[r, r^{\prime}\right]$ fixed, there exists an open neighbourhood $W[t] \subseteq G$ of $e$, as well as $U[t] \subseteq \mathfrak{g}$ open with $0 \in U[t]$, such that

$$
\alpha\left((g, X),\left(g^{\prime}, X^{\prime}\right)\right)<\varepsilon \quad \forall(g, X),\left(g^{\prime}, X^{\prime}\right) \in[\mu(t) \cdot W[t]] \times[\psi(t)+U[t]]
$$

holds. We choose

a) $V[t] \subseteq G$ open with $e \in V[t]$ and $V[t] \cdot V[t] \subseteq W[t]$.

b) $O[t] \subseteq \mathfrak{g}$ open with $0 \in O[t]$ and $O[t]+O[t] \subseteq U[t]$.

c) $J[t] \subseteq \mathbb{R}$ open with $t \in J$, such that for $D[t]:=J[t] \cap\left[r, r^{\prime}\right]$, we have

$$
\mu(D[t]) \subseteq \mu(t) \cdot V[t] \subseteq \mu(t) \subseteq W[t] \quad \text { and } \quad \psi(D[t]) \subseteq \psi(t)+O[t] \subseteq \psi(t)+U(t) .
$$

Since $\left[r, r^{\prime}\right]$ is compact, there exist $t_{0}, \ldots, t_{n} \in\left[r, r^{\prime}\right]$, such that $\left[r, r^{\prime}\right] \subseteq D_{0} \cup \ldots \cup D_{n}$ holds.

- We define $V:=V\left[t_{0}\right] \cap \ldots \cap V\left[t_{n}\right]$.

Since $G$ is Mackey k-continuous, there exists some $N_{\varepsilon} \in \mathbb{N}$ with

$$
\int_{r}^{\bullet} \phi_{n} \in \int_{r}^{\bullet} \phi \cdot V \quad \forall n \geq N_{\varepsilon} .
$$

- We define $O:=O\left[t_{0}\right] \cap \ldots \cap O\left[t_{n}\right]$.

Since $\left\{\psi_{\alpha}\right\}_{\alpha \in I} \rightarrow_{\mathfrak{n} .0} \psi$ holds, there exists $\alpha_{\varepsilon} \in I$ with

$$
\left(\psi_{\alpha}(t)-\psi(t)\right) \in O \quad \forall t \in\left[r, r^{\prime}\right], \alpha \geq \alpha_{\varepsilon} .
$$

Then, for $\tau \in D_{p}$ with $0 \leq p \leq n$, as well as $n \geq N_{\varepsilon}$ and $\alpha \geq \alpha_{\varepsilon}$, we obtain from (173), (174), as well as (72) for $t \equiv t_{p}$ there that

- $\mu\left(t_{p}\right)^{-1} \cdot \int_{r}^{\tau} \phi_{n}=\left(\mu\left(t_{p}\right)^{-1} \cdot \mu(\tau)\right) \cdot\left(\left[\int_{r}^{\tau} \phi\right]^{-1}\left[\int_{r}^{\tau} \phi_{n}\right]\right) \in V \cdot V \subseteq W\left[t_{p}\right]$,

- $\psi_{\alpha}(\tau)-\psi\left(t_{p}\right)=\left(\psi_{\alpha}(\tau)-\psi(\tau)\right)+\left(\psi(\tau)-\psi\left(t_{p}\right)\right) \in O+O \subseteq U\left[t_{p}\right]$.

The claim is thus clear from (71) and (72).

Proof of Lemma 18. For each $\chi, \chi^{\prime} \in \mathfrak{D}_{\left[r, r^{\prime}\right]}^{k}$, we have, cf. (33)

$$
\xi\left(\chi, \chi^{\prime}\right)=\mathrm{d}_{\left(\int \chi, e\right)} \mathrm{m}\left(0, \widehat{\Gamma}\left(\chi, \chi^{\prime}\right)\right) \quad \text { for } \quad \Gamma \equiv \operatorname{Ad}(\operatorname{inv}(\cdot), \cdot) ;
$$

so that (69) holds by Lemma 24, because the Lie group multiplication is smooth. 


\section{A.5 Proof of Equation (56)}

In this appendix, we show

$$
\int \phi=\exp \left(\int \phi(s) \mathrm{d} s\right) .
$$

Proof of Equation (56). We fix $I \equiv\left(\iota, \iota^{\prime}\right)$ with $\iota<r<r^{\prime}<\iota^{\prime}$, define $\psi \in C^{0}\left(\left[\iota, \iota^{\prime}\right], \mathfrak{g}\right)$ by

$$
\left.\psi\right|_{[\iota, r)}:=\left.\phi(r) \quad \psi\right|_{\left[r, r^{\prime}\right]}:=\left.\phi \quad \psi\right|_{\left(r^{\prime}, \iota^{\prime}\right]}:=\phi\left(r^{\prime}\right) ;
$$

and observe that 18

$$
\mathfrak{X}: I \rightarrow \operatorname{dom}[\exp ], \quad x \mapsto-(r-\iota) \cdot \phi(r)+\int_{\iota}^{x} \psi(s) \mathrm{d} s
$$

fulfills the presumptions in Corollary 6, with

$$
\exp \left(\int_{r}^{\bullet} \phi(s) \mathrm{d} s\right)=\left.\alpha\right|_{\left[r, r^{\prime}\right]} \quad \text { for } \quad \alpha:=\exp \circ \mathfrak{X} .
$$

By Corollary [6, we thus have $\alpha \in C^{1}(I, G)$, with

$$
\begin{aligned}
\delta^{r}(\alpha)(x) & =\left.\frac{\mathrm{d}}{\mathrm{d} h}\right|_{h=0} \alpha(x+h) \cdot \alpha(x)^{-1} \\
& =\left.\frac{\mathrm{d}}{\mathrm{d} h}\right|_{h=0} \alpha(x)^{-1} \cdot \alpha(x+h) \\
& =\mathrm{d}_{\exp (\mathfrak{X}(x))} \mathrm{L}_{\exp (-\mathfrak{X}(x))}\left(\left.\frac{\mathrm{d}}{\mathrm{d} h}\right|_{h=0} \alpha(x+h)\right) \\
& =\left(\mathrm{d}_{\exp (\mathfrak{X}(x))} \mathrm{L}_{\exp (-\mathfrak{X}(x))} \circ \mathrm{d}_{e} \mathrm{~L}_{\exp (\mathfrak{X}(x))}\right)\left(\int_{0}^{1} \operatorname{Ad}_{\exp (-s \cdot \mathfrak{X}(x))}(\dot{\mathfrak{X}}(x)) \mathrm{d} s\right) \\
& =\int_{0}^{1} \dot{\mathfrak{X}}(x) \mathrm{d} s=\dot{\mathfrak{X}}(x)=\psi(x)=\phi(x)
\end{aligned}
$$

for each $x \in\left[r, r^{\prime}\right]$. Here, we have used in the second-, and the fifth step that $G$ is abelian.

\section{A.6 Proof of Lemma 22}

In this appendix, we prove

Lemma 22. Suppose that $F$ is metrizable; and let $\left\{X_{n}\right\}_{n \in \mathbb{N}} \subseteq F$ be a sequence with $\left\{X_{n}\right\}_{n \in \mathbb{N}} \rightarrow$ $X \in F$. Then, we have $\left\{X_{n}\right\}_{n \in \mathbb{N}} \rightarrow_{\mathfrak{m}} X$.

Proof. We choose $\mathfrak{H}:=\{\mathfrak{q}[m] \mid m \in \mathbb{N}\} \subseteq \mathfrak{Q}$ as in Lemma 21]3) and let $\mathfrak{l}: \mathbb{N} \rightarrow \mathbb{N}$ be strictly increasing with

$$
\mathfrak{q}[m]\left(X-X_{n}\right) \leq \frac{1}{m} \quad \forall n \geq \mathfrak{l}_{\mathfrak{q}[m]}:=\mathfrak{l}(m), m \in \mathbb{N} .
$$

- We define $\lambda_{n}:=\frac{1}{m}$ for each $n \in \mathbb{N}$ with $\mathfrak{l}(m) \leq n<\mathfrak{l}(m+1)$; and observe that $\lim _{n} \lambda_{n}=0$ holds.

- For $m, d, n \in \mathbb{N}$ with $\mathfrak{l}(m+d) \leq n<\mathfrak{l}(m+d+1)$, we obtain

$$
\mathfrak{q}[m]\left(X-X_{n}\right) \leq \mathfrak{q}[m+d]\left(X-X_{n}\right) \stackrel{(75)}{\leq} \frac{1}{m+d}=\lambda_{n} .
$$

This shows that $\mathfrak{q}[m]\left(X-X_{n}\right) \leq \lambda_{n}$ holds for each $n \geq \mathfrak{l}_{\mathfrak{q}[m]}=\mathfrak{l}(m)$; thus, $\left\{X_{n}\right\}_{n \in \mathbb{N}} \rightarrow_{\mathfrak{m}} X$.

\footnotetext{
${ }^{18}$ Recall the last statement in Sect. 2.2 .3 for the fact that $\operatorname{im}[\mathfrak{X}] \subseteq \operatorname{dom}[\exp ]$ holds.
} 


\section{A.7 Proof of Lemma 23}

In this appendix, we prove

Lemma 23. If $F$ is metrizable, then $C^{k}([0,1], F)$ is metrizable for each $k \in \mathbb{N} \sqcup\{$ lip, $\infty, c\}$.

Proof. Let $\{\mathfrak{q}[m] \mid m \in \mathbb{N}\} \subseteq \mathfrak{Q}$ be as in Lemma 21]3), For each $m \in \mathbb{N}$, we define

$$
\mathrm{s}[\operatorname{lip}, m]:=\operatorname{lip} \quad \mathrm{s}[\mathrm{c}, m]:=0 \quad \mathrm{~s}[\infty, m]:=m \quad \text { as well as } \quad \mathrm{s}[k, m]:=k \quad \forall k \in \mathbb{N} \text {. }
$$

Moreover, for each $k \in \mathbb{N} \sqcup\{$ lip, $\infty, \mathrm{c}\}$, we let

$$
\mathfrak{s}[k, m]:=\mathfrak{q}[m]_{\infty}^{\mathrm{s}[k, m]} \quad \forall m \in \mathbb{N} \quad \text { as well as } \quad \mathfrak{S}_{k}:=\{\mathfrak{s}[k, m] \mid m \in \mathbb{N}\} .
$$

Let now $k \in \mathbb{N} \sqcup\{\operatorname{lip}, \infty, \mathrm{c}\}, \mathfrak{q} \in \mathfrak{Q}, \mathrm{s} \preceq k$ be fixed. By Lemma 20, there exist $c>0, \ell \in \mathbb{N}$ with $\mathfrak{q} \leq c \cdot \mathfrak{q}[\ell]$. We define

$$
m:= \begin{cases}\ell & \text { for } k \in \mathbb{N} \sqcup\{\text { lip }, \mathrm{c}\}, \\ \max (\mathrm{s}, \ell) & \text { for } k=\infty,\end{cases}
$$

observe that $\mathfrak{q} \leq c \cdot \mathfrak{q}[m]$ as well as $\mathrm{s} \leq \mathrm{s}[k, m]$ holds; and obtain

$$
\mathfrak{q}_{\infty}^{\mathrm{s}} \leq c \cdot \mathfrak{q}[m]_{\infty}^{\mathrm{s}[k, m]}=c \cdot \mathfrak{s}[k, m] .
$$

Then, Lemma 20 shows that $\mathfrak{S}_{k}$ is a fundamental system; and, since $\mathfrak{S}_{k}$ is countable, the claim follows from Lemma 21.

\section{References}

[1] J. J. Duistermaat and J. A. C. Kolk, Lie Groups, Universitext, Springer, Berlin, 2000.

[2] H. Glöckner: Infinite-dimensional Lie groups without completeness restrictions, in: Geometry and Analysis on Finite- and Infinite-dimensional Lie Groups (Bȩdlewo 2000), Banach Center Publ. 55, Polish Academy of Sciences, Warsaw (2002), 4359.

[3] H. Glöckner: Regularity properties of infinite-dimensional Lie groups, and semiregularity, preprint (2012), https://arxiv.org/abs/1208.0715.

[4] H. Glöckner: Measurable regularity properties of infinite-dimensional Lie groups, preprint (2015), https://arxiv.org/abs/1601.02568.

[5] H. Glöckner, K.-H. Neeb: When unit groups of continuous inverse algebras are regular Lie groups, Studia Math. 211 (2012), no. 2, 95-109.

[6] R. Hamilton: The inverse function theorem of Nash and Moser, Bull. Amer. Math. Soc. (N. S.) 7 (1982), no. 1, 65-222.

[7] M. Hanusch: Regularity of Lie groups, preprint (2017), https://arxiv.org/abs/1711.03508v2.

[8] M. Hanusch: The strong Trotter property for locally $\mu$-convex Lie Groups, preprint (2018), https://arxiv.org/abs/1802.08923v1.

[9] M. Hanusch: The regularity problem for asymptotic estimate Lie groups, preprint (2018), https://arxiv.org/abs/1804.10956v1.

[10] H. Jarchow: Locally convex spaces, B.G. Teubner, Stuttgart, 1981. 
[11] A. Kriegl and P.W. Michor: Regular infinite-dimensional Lie groups, J. Lie Theory 7 (1997), no. 1, 61-99.

[12] A. Kriegl and P. W. Michor: The Convenient Setting of Global Analysis, Math. Surveys Monogr. 53, American Mathematical Society, Providence, 1997.

[13] T. Marquis and K.-H. Neeb: Half-Lie groups, Transformation Groups, 23 (2018), no. 3, 801840.

[14] R. Meise and D. Vogt: Introduction to Functional Analysis, Oxf. Grad. Texts Math. 2, Oxford University, New York, 1997.

[15] J. Milnor: Remarks on infinite-dimensional Lie groups, in: Relativity, Groups and Topology. II (Les Houches 1983), North-Holland, Amsterdam (1984), 10071057.

[16] K.-H. Neeb: Towards a Lie theory of locally convex groups, Jpn. J. Math. 1 (2006), no. 2, 291-468.

[17] K.-H. Neeb: Monastir summer school. Infinite-dimensional Lie groups, Preprint 2433, TU Darmstadt, 2006.

[18] K.-H. Neeb: Infinite-dimensional groups and their representations, in: Infinite Dimensional Kähler Manifolds (Oberwolfach 1995), DMV Sem. 31, Birkhäuser, Basel (2001), 131178.

[19] K.-H. Neeb and H. Salmasian: Differentiable vectors and unitary representations of Fréchet-Lie supergroups, Math. Z. 275 (2013), no. 1-2, 419-451.

[20] H. Omori, Y. Maeda, A. Yoshioka and O. Kobayashi: On regular FrchetLie groups. IV. Definition and fundamental theorems, Tokyo J. Math. 5 (1982), no. 2, 365398. 